Review

\title{
Development of Pharmaceutical VOCs Elimination by Catalytic Processes in China
}

\author{
Lilong Zhou ${ }^{1, *}$, Chen Ma ${ }^{1}$, Jonathan Horlyck ${ }^{2}$, Runjing Liu ${ }^{1}$ and Jimmy Yun ${ }^{1,2,3, *}$ \\ 1 College of Chemical and Pharmaceutical Engineering, Hebei University of Science and Technology, \\ Shijiazhuang 050018, China; machen33@126.com (C.M.); liurj@hebust.edu.cn (R.L.) \\ 2 School of Chemical Engineering, The University of New South Wales, Sydney NSW 2052, Australia; \\ j.horlyck@outlook.com \\ 3 Qingdao International Academician Park Research Institute, Qingdao 266000, China \\ * Correspondence: 1lzhou@hebust.edu.cn (L.Z.); jimmy.yun@unsw.edu.au (J.Y.)
}

Received: 26 May 2020; Accepted: 9 June 2020; Published: 13 June 2020

\begin{abstract}
As a byproduct of emerging as one of the world's key producers of pharmaceuticals, China is now challenged by the emission of harmful pharmaceutical VOCs. In this review, the catalogue and volume of VOCs emitted by the pharmaceutical industry in China was introduced. The commonly used VOC removal processes and technologies was recommended by some typical examples. The progress of catalytic combustion, photocatalytic oxidation, non-thermal plasma, and electron beam treatment were presented, especially the development of catalysts. The advantages and shortages of these technologies in recent years were discussed and analyzed. Lastly, the development of VOCs elimination technologies and the most promising technology were discussed.
\end{abstract}

Keywords: VOCs; catalytic combustion; China; elimination technology; pharmaceutical industry

\section{Introduction}

Recently, the Chinese public has become increasingly concerned about the levels of chemical air pollution present in the form of haze. A main contributing factor to this pollution is the release of volatile organic compounds (VOCs) from the industry. VOCs are organic compounds with boiling points in the range of $50-260^{\circ} \mathrm{C}$ at atmospheric pressure or with a Reid vapor pressure of over $10.3 \mathrm{~Pa}$ at room temperature $(293.15 \mathrm{~K})$ and atmosphere pressure $(101.325 \mathrm{kPa})[1,2]$. The pharmaceutical industry is a major source of these VOCs [3,4], which can have serious ramifications, such as toxicity, carcinogenesis, mutagenesis, photochemical pollution, haze, and fog [5-9]. The pharmaceutical industry has developed greatly in China, as the production of bulk drug intermediates and Chinese patent drugs increased from 205,070 to 340,830 kilotons, and 112,890 to 374,600 kilotons in the past 10 years (2007-2016), respectively [10]. Meanwhile, the amounts of released VOCs increased from about 174.8 to $393.2 \mathrm{kt}$ in the pharmaceutical industry [11,12]. The guiding emission standards of VOCs went into effect on 1 January, 2018 in China, which led to new imputes to upgrade VOCs elimination equipment and technologies in factories across China [13].

\section{Catalogue and Emission Amounts of VOCs in the China Pharmaceutical Industry}

\subsection{Catalogue of Pharmaceutical VOCs in China}

Medicines in China are mainly produced in six ways, biological fermentation, chemical synthesis, extraction, coagulation preparation, bioengineering, and treatment of traditional Chinese medicine $[4,14]$. The different methods produce varying levels of pollution, with the order of emitted VOCs being biological fermentation $>$ chemical synthesis $>$ extraction $>$ bioengineering $>$ treatment of 
traditional Chinese medicine $>$ coagulation preparation [15]. All these processes can be plagued by the need for high volumes of solvents, the consumption of large quantities of complex organic precursors, or the production of volatile byproducts. Some of the volatile organic compounds used or produced in these processes may be released into the atmosphere, which causes air pollution. The released VOCs primarily include alkanes, alcohols, ketones, aromatic hydrocarbons, halohydrocarbons, amines, esters, ether, aldehyde, carboxylic acid, and sulfur containing organic compounds $[4,11,12,14,15]$.

Biological fermentation is often used to produce antibiotics, vitamins, and amino acids, via processes which include fermentation, separation, purification, and refinement $[16,17]$. The primary source of emitted VOCs are the solvents used in these processes, especially in the separation and purification steps. Additionally, $\mathrm{H}_{2} \mathrm{~S}$ is also produced as a byproduct in some fermentation processes.

The chemical synthesis technology is usually used for the production of medicine which can be used for prevention, cure, and diagnosis of the disease [18,19]. It contains the units to synthesize intermediates from raw materials, modify the structure of intermediates, purify the products, and dry the final products. The VOCs from this process comprise solvents and unreacted intermediates, which are more complex and often harder to be eliminated than those produced from the biological fermentation. These VOCs include heptane, toluene, xylene, methanol, n-propyl alcohol, isopropanol, phenol, aminomethane, dimethylamine, aminobenzene, cyclohexylamine, triethylamine, butyraldehyde, acetone, chloroform, chlorobenzene, etc.

Extraction involves the use of physical, chemical, and biological methods to separate a substance from a mixture. In the case of the pharmaceutical industry, extraction is carried out to separate organic compounds of interest from liquid solvents $[16,20]$. As a result, extraction processes require a large supply of solvents, including toluene, naphtha, methanol, ethanol, isopropanol, phenol, acetone, ether, acetic ether, diethylamine, dichloromethane, dichloloethane, chloroform, etc. VOCs are released during extraction mainly from the organic solvents used within the process.

The bioengineering method is a new way to produce some new medicines, via processes such as cloning antibodies, genetic engineering drugs, and genetic engineering vaccines [21]. The VOCs released from this process are similar to those of biological fermentation. These VOCs come from the solvents used within the process and as byproducts of certain reactions. They include n-hexane, methanol, ethanol, formaldehyde, acetaldehyde, formic acid, propanediol, acetone, aminoehtyl alcohol, acetonitrile, acetic acid, acetocaustin, N, N-dimethylformamide, phenol, butanone, 4-methyl-2-pentone, n-propyl alcohol, isopropanol, n-pentanol, isopropyl ether, isobutyl aldehyde, etc.

Another process of pharmaceutical production is the manufacture of traditional Chinese medicines or certain Chinese patent drugs [22]. As this is a traditional process which involves the use of only limited organic solvents to treat the natural animals and plants, the production of VOCs is limited. Hence, this process only emits a spot of VOCs, $\mathrm{SO}_{2}$, and smoke.

Coagulation preparation involves the formation of larger particle agglomerates from fine particle suspensions. In the synthesis of pharmaceuticals, it is the process of mixing the active ingredients with helper constituents (often called coagulants) to produce a drug which has the desired particle size. The physical nature of the coagulation process means that the waste produced via this method is primarily a solid particle with little notable VOC production [4,14-16,22].

\subsection{The Guiding Emission Standards of VOCs in China}

The guiding emission standards of VOCs include six kinds of VOCs on the boundary of factories (Table 1) and four pollutant classifications with a total of 16 compounds in the areas surrounding workshops and installations (Table 2) [13]. These parameters are determined in $1 \mathrm{~h}$ by $3 \sim 4$ samples for the average value. They are detected by the portable instruments or GC. These target VOCs are carcinogenic, odorous, and harmful organic compounds, which are mainly used as solvents and raw materials. 
Table 1. The extreme emission value of volatile organic compounds (VOCs) on the boundary of factories and the highest concentration of selected VOCs allowed in $1 \mathrm{~h}$ for employees [13].

\begin{tabular}{|c|c|c|}
\hline Pollutants & Limiting Value $\left(\mathrm{mg} \cdot \mathrm{m}^{-3}\right)$ & $\begin{array}{l}\text { The Highest Concentration of Selected } \\
\text { VOCs Allowed in } 1 \mathrm{~h} \text { for Employees } \\
\qquad\left(\mathrm{mg} \cdot \mathrm{m}^{-3}\right)\end{array}$ \\
\hline Benzene & 0.4 & 6 \\
\hline Formaldehyde & 0.2 & 0.1 \\
\hline Trichloroethylene ${ }^{a}$ & 0.1 & 30 \\
\hline Dimethyl sulfate $^{a}$ & 0.5 & 0.5 \\
\hline Dichloromethane $^{\text {a }}$ & 4.0 & 0.5 \\
\hline Non-methane organic compounds & 4.0 & - \\
\hline Ozone & 20 & 0.26 \\
\hline
\end{tabular}

a. They will be put into effect after the publication of national standards.

Table 2. The limiting value of specific pollutants in workshops and installations [13].

\begin{tabular}{|c|c|c|c|}
\hline \multirow{2}{*}{ Classification } & \multirow{2}{*}{ Pollutant } & \multicolumn{2}{|c|}{ The Limiting Values $\left(\mathrm{mg} \cdot \mathrm{m}^{-3}\right)$} \\
\hline & & General Area & Key Area \\
\hline \multirow{3}{*}{ Carcinogens } & Trichloroethylene $^{\mathrm{a}}$ & 1 & 1 \\
\hline & Benzene & 4 & 4 \\
\hline & Formaldehyde & 5 & 5 \\
\hline \multirow{5}{*}{ Toxic substances } & Phosgene & 0.5 & 0.5 \\
\hline & $\mathrm{HCN}$ & 1.9 & 1.9 \\
\hline & Acrolein & 3 & 3 \\
\hline & Methyl sulfate ${ }^{a}$ & 5 & 5 \\
\hline & $\mathrm{Cl}_{2}$ & 5 & 5 \\
\hline \multirow{4}{*}{$\begin{array}{l}\text { Photochemically active } \\
\text { substances }\end{array}$} & Toluene & 25 & 15 \\
\hline & xylene & 40 & 20 \\
\hline & Dimethyl sulfoxide $^{a}$ & 100 & 50 \\
\hline & Butylene oxide $^{a}$ & 100 & 50 \\
\hline \multirow{4}{*}{ Other } & $\mathrm{NH}_{3}$ & 20 & 10 \\
\hline & $\mathrm{HCl}$ & 20 & 10 \\
\hline & $\mathrm{CH}_{3} \mathrm{OH}$ & 50 & 30 \\
\hline & $\mathrm{CH}_{2} \mathrm{Cl}_{2}{ }^{\mathrm{a}}$ & 75 & 45 \\
\hline
\end{tabular}

a. They will be put into effect after the publication of national standards.

\subsection{VOCs Emissions in the Chinese Pharmaceutical Industry}

China is the second largest producer of pharmaceutical products, only behind the United States of America. There are more than 1300 kinds of drug intermediates, 30 types of medicaments, and over 4500 pharmaceutical products made in China [23]. According to previous studies, the total VOCs emissions scaled linearly with the amount of final pharmaceutical products. [24]. The results showed that about $0.55 \mathrm{~kg}$ of VOCs were discharged to the atmosphere for the production of each $1 \mathrm{~kg}$ of final drug products.

The total VOCs emissions from the pharmaceutical industry increased by over $120 \%$ from about $174.8 \mathrm{kt}$ in 2007 to $393.2 \mathrm{kt}$ in 2016 (Figure 1) [12]. Although the VOCs emitted from the pharmaceutical industry only account for approximately $1.1 \%$ of China's total VOCs emissions, the absolute emission amount is very large. VOCs emitted from the pharmaceutical industry are potentially more harmful to human beings and ecosystems than the VOCs emitted from other sources. Compared with other emission sources, such as decoration, oil extraction and refining, catering, shoemaking and furniture manufacturing, the VOCs from pharmaceutical industries are more diverse with higher local concentrations and are harder to be eliminated. 


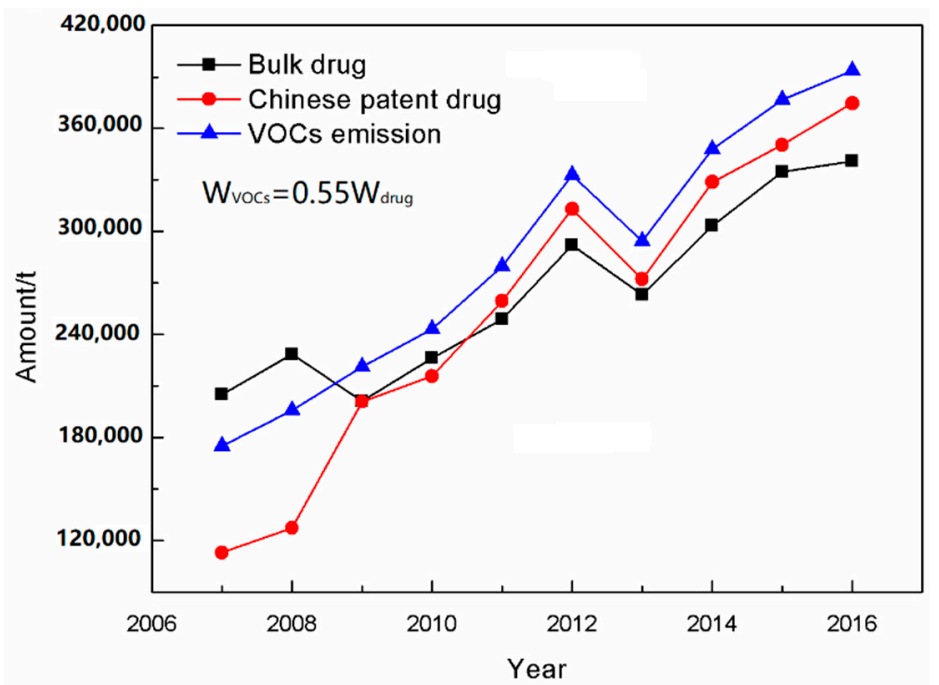

Figure 1. The production of bulk drugs and Chinese patent drugs and VOCs emissions from 2007 to 2016.

\section{The Developing Technologies to Dispel VOCs}

\section{VOCs Elimination Technologies used in China's Pharmaceutical Industry}

VOCs elimination technologies used in China were originally developed with the aim of recycling organic compounds to reduce cost, but recently the use and implementation of these technologies are targeted at minimizing the environmental impacts of VOCs. Technologies applied in the elimination of VOCs in China include condensation, absorption, adsorption, membrane purification, incineration, catalytic combustion, and the non-thermal plasma process (Figure 2) [15,25-27]. These methods are applied according to different working conditions, such as temperature and pressure, depending upon the VOCs targeted for removal. They also have distinct advantages and disadvantages. The development of these VOCs elimination technologies will be briefly introduced and discussed in this review. It is important to note that a wide range of VOCs which are often produced in a single waste stream and the various treatment technologies have different efficacies for the removal of certain VOCs. In fact, multiple technologies are often combined to eliminate the VOC mixtures from the waste streams.

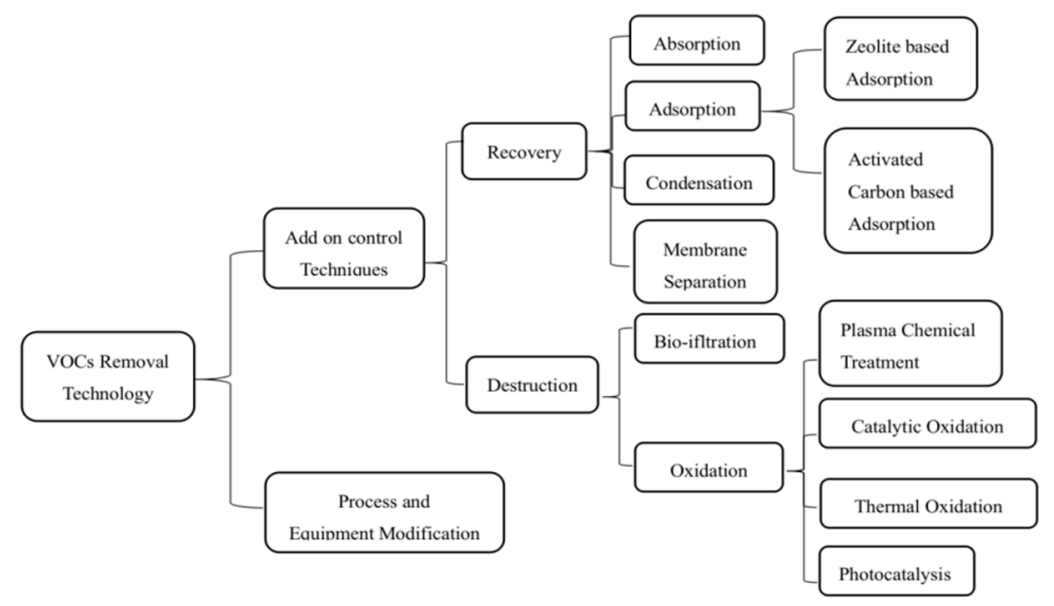

Figure 2. Classification of VOCs control techniques. Reprinted with permission from [26], 2000, Elsevier Ltd.

Condensation is the conversion of gas phase VOC mixtures with different vapor pressures to liquid via a decrease in temperature [28]. This technology is often used to recycle the solvents used in 
pharmaceutical production, with the key advantages that equipment requirements and operation of the condensation process are simple. It makes the cost of condensation lower than other technologies. Another advantage is that the gas produced from condensation is pure. Hence, condensation is often used as the first procedure to treat the waste gas from a pharmaceutical workshop before incineration and absorption to reduce the load on more complex and expensive downstream technologies. Water and air are the most commonly used cooling mediums for the condensation process, but ice, cold salt solutions and organic mediums have been used where cooling temperatures below $10{ }^{\circ} \mathrm{C}$ are required, such as the $\mathrm{CaCl}_{2}$ solution, $\mathrm{NaCl}$ solution, and ethylene glycol aqueous solution [4]. The condensation efficiency is sensitive to the temperature and pressure and is suitable for the removal of high concentration VOCs which exist as liquefied at moderate temperatures $[29,30]$.

The removal of VOCs via adsorption involves the use of porous materials, while absorption utilizes solvents. The porous materials used in adsorption need a high absorption capacity, large surface area, good pore structure, stable chemical properties, high physical strength, and tolerance of acidic/basic conditions. The adsorption materials commonly used in this technology include activated carbon, porous silica, zeolite, and porous resin [31-35]. The solvents commonly used in the absorption of VOCs are water, acid solution, alkali solution, and other organic compounds [36]. The components used in the adsorption process include spray columns, filled towers, columns of trays, and washing apparatuses.

Both the adsorption and absorption methods have a high VOC removal efficiency and can almost completely remove VOCs from waste gas with low energy consumption (Figure 3). They can be used to recycle organic solvents and valuable compounds while remaining economically feasible. This technology is often used for the treatment of a large flow of waste gas with low VOC concentration in processes such as fix bed adsorption, moving bed adsorption, fluid-bed adsorption, and pressure swing adsorption [37-39]. Disadvantages of the adsorption and absorption process are also noteworthy, such as huge equipment requirements, complex procedures, and the need for desorption and regeneration of saturated absorbents. Due to its high overall VOC removal efficiency, this technology is commonly used in many pharmaceutical factories.

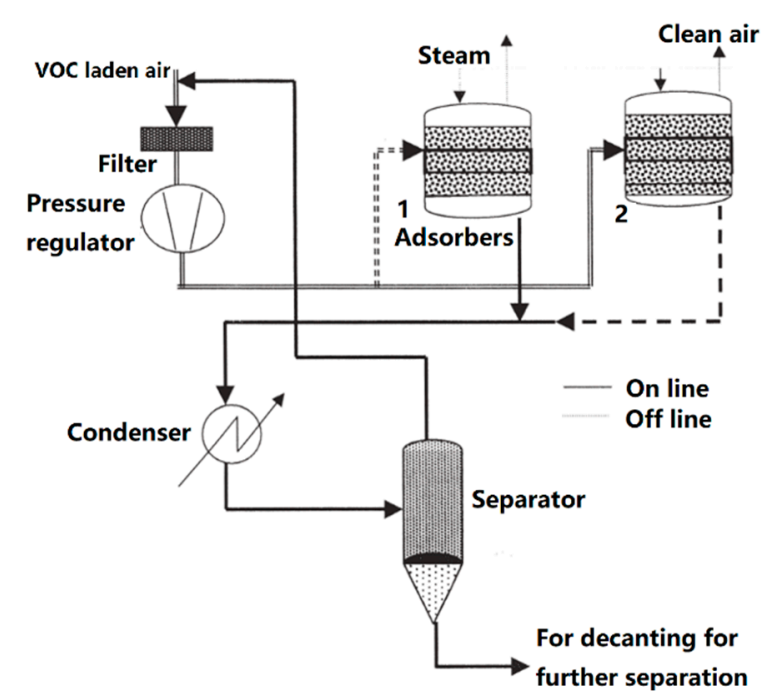

Figure 3. A typical activated carbon VOC removal (solvent recovery) plant. Reprinted with permission from [26], 2000, Elsevier Ltd.

Incineration is another widely used technology in pharmaceutical factories to eliminate VOCs (Figure 4). If VOC recycling is not technologically or financially feasible, incineration is a suitable method to completely eliminate VOCs [40]. Incineration is carried out by burning VOCs in a stove or kiln. Ideally, incineration results in the conversion of $\mathrm{VOCs}$ into $\mathrm{CO}_{2}$ and $\mathrm{H}_{2} \mathrm{O}$ in an efficient, simple, and safe manner. However, it has multiple shortcomings. If the VOCs concentration is too low to support the incineration, additional fuel is needed, which increases the running cost. Additionally, 
some VOCs are less suitable for incineration, because the incomplete combustion of halogenated and other harmful VOCs can result in the release of toxic chemicals such as dioxin, $\mathrm{NO}_{\mathrm{x}}$, and $\mathrm{CO}$ [40].

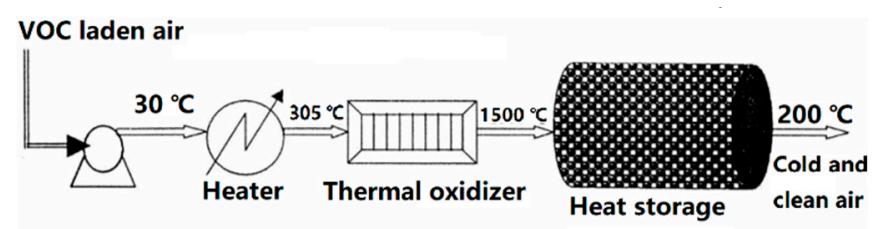

(a)

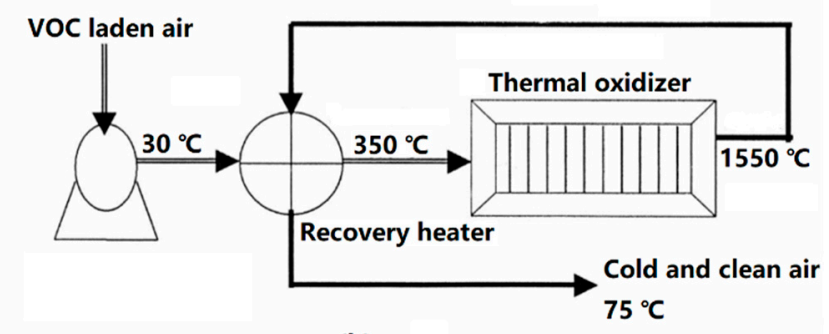

(b)

Figure 4. Schemes of thermal oxidation. (a) Regenerative thermal oxidation; (b) recuperative thermal oxidation. Reprinted with permission from [26], 2000, Elsevier Ltd.

To overcome the shortages of the incineration process for VOC destruction, the catalytic combustion technology was developed. In this process, VOCs are decomposed over catalysts at a low temperature (lower than $500{ }^{\circ} \mathrm{C}$, Figures 5 and 6) [1,2,41-44]. The key factor which governs the catalytic combustion process is the activity of catalysts. Various kinds of catalysts have been used for the catalytic combustion of VOCs, such as noble metal catalysts, transition metal oxides, perovskite catalysts, and concentrated oxidation catalysts. The advantages for this technology are low operation temperature, decreased energy input requirements, high VOC removal efficiency, and minimal generation of toxic byproducts. Catalytic combustion is suitable for the treatment of waste streams containing VOCs across a wide range of concentrations. The main disadvantages of catalytic combustion are high investment requirements for equipment, short catalyst lifetime, and the need for process-specific designs, which are tailored to the waste stream. Nonetheless, the development of viable materials for the catalytic combustion process is still a hotspot for catalysis science.

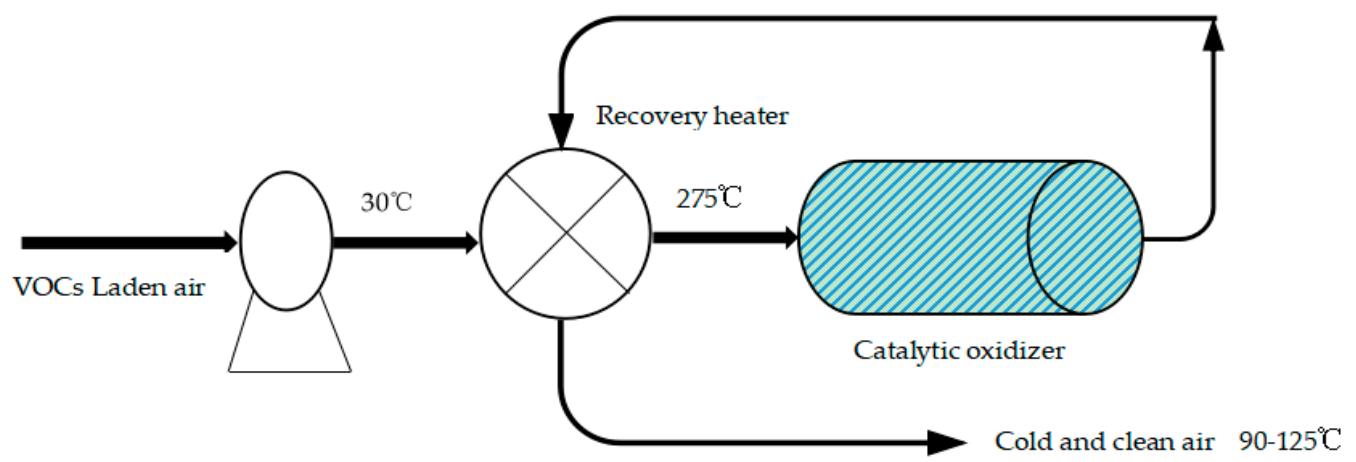

Figure 5. Scheme of catalytic oxidation. Reprinted with permission from [26], 2000, Elsevier Ltd.

The mechanism of catalytic combustion is considered by three types, with these being the Mars-Van Krevelen (MVK) model, Langmuir-Hinshelwood (LH) model, and Eley -Rideal (ER) model. In the Mars-Van Krevelen model, the VOCs molecules are initially adsorbed on the active sites, upon which they react with the oxygen species within the catalyst and are decomposed. Then, the reduced catalyst is re-oxidized by the supply of oxygen to the reactor. In the Langmuir-Hinshelwood model, the adsorbed VOCs molecules react directly with the adsorbed oxygen molecules, all occurring on the 
catalyst surface. In the Elay-Rideal mechanism, the adsorbed oxygen reacts with the VOCs molecules in the gas phase. The reaction pathway which follows depends on both the catalyst materials and the target VOCs in individual systems.

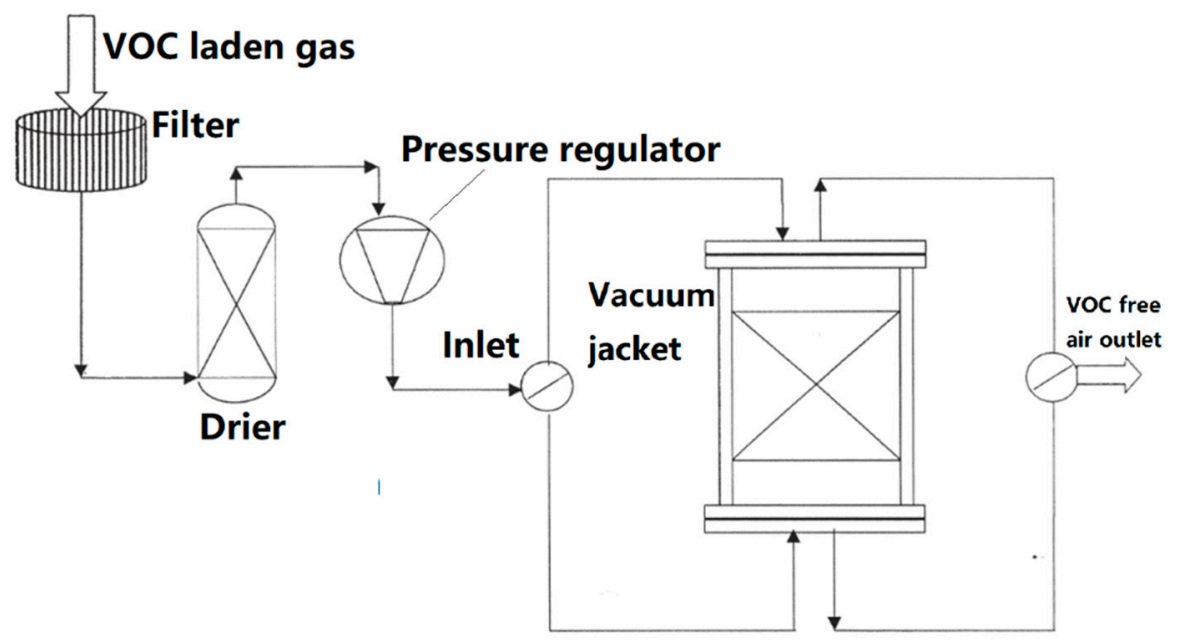

Figure 6. Schematic diagram of a reverse flow reactor. Reprinted with permission from [26], 2000, Elsevier Ltd.

Biodegradation is a widely used process for the treatment of pharmaceutical wastewater (Figure 7) [45,46]. It also can be applied to the treatment VOCs in the gas phase, especially for low concentration VOCs which are suitable for the growth of microorganisms. This process works with using the VOCs as a feedstock for the microorganisms, where they are converted to cytoplasm, $\mathrm{CO}_{2}$, and $\mathrm{H}_{2} \mathrm{O}$. The sulfur and nitrogen elements in VOCs can be transformed to $\mathrm{H}_{2} \mathrm{~S}$, nitrate, or $\mathrm{N}_{2}$ at moderate temperatures. However, VOCs emitted from the pharmaceutical process often contain aromatics or halogens, which would poison the microorganism, rendering this method largely unviable in the abatement of pharmaceutical VOCs.

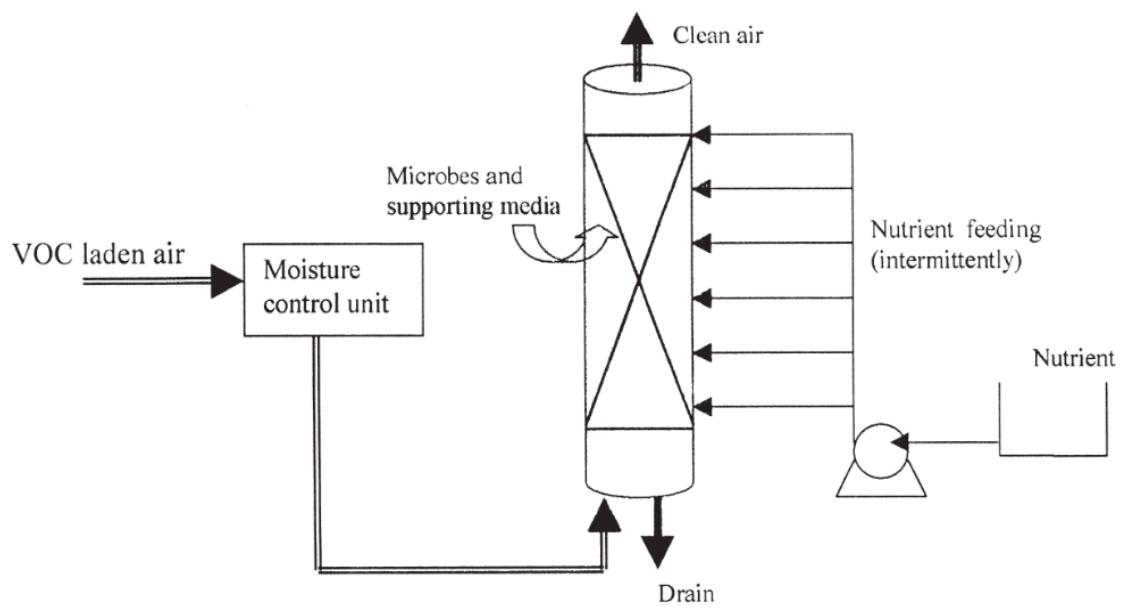

Figure 7. A simple schematic sketch of a bio-filtration system. Reprinted with permission from [26], 2000, Elsevier Ltd.

Non-thermal plasma is also a commonly used technology for the elimination of VOCs [47-49]. Free electrons and radicals formed during the plasma process react with VOCs and lead to the degradation of VOCs to $\mathrm{CO}_{2}$ and $\mathrm{H}_{2} \mathrm{O}$. There are some advantages of the non-thermal plasma technology, such as a low press drop across the reactor, compact size, and simple equipment structure. The process can be started immediately without warm up and can treat VOCs with solid particles and liquid drops. 
The non-thermal plasma technology can be combined with catalytic combustion to achieve a superior abatement of pharmaceutical VOCs.

The application of different VOCs elimination technologies depends on the factors such as the range and concentration of VOCs present in the waste stream, the volume of waste stream, and the funds available for installation and running costs. Jie Hao et al. summarized the scope of application for different VOCs elimination technologies (Table 3) [50]. Condensation and adsorption recycling are suitable for recycling VOCs. Catalytic combustion and incineration can remove the VOCs with a moderate concentration (3000-1/4 LEL) at high temperatures, while biodegradation and non-thermal plasma are suitable for abatement of low concentration VOCs at moderate temperatures. Hence, when selecting the efficient and economic VOCs abatement technologies, the scope of application for different technologies needs to be considered.

Table 3. VOCs elimination techniques and their operating conditions [50].

\begin{tabular}{cccc}
\hline Abatement Technologies & $\begin{array}{c}\text { VOC Concentration } \\
\left(\mathbf{m g} \cdot \mathbf{m}^{-3}\right)\end{array}$ & Discharge Rate $\left(\mathbf{m}^{\mathbf{3}} \cdot \mathbf{h}^{-\mathbf{1}}\right)$ & Temperature $\left({ }^{\circ} \mathbf{C}\right)$ \\
\hline Adsorption recycling & $100-1.5 \times 10^{4}$ & $<6 \times 10^{4}$ & $<45$ \\
Preheated catalytic & $3000-1 / 4 \mathrm{LEL}^{*}$ & $<4 \times 10^{4}$ & $<500$ \\
combustion & $1000-1 / 4 \mathrm{LEL}$ & $<4 \times 10^{4}$ & $<500$ \\
Thermal storage catalytic & $3000-1 / 4 \mathrm{LEL}$ & $<4 \times 10^{4}$ & $>700$ \\
combustion & $1000-1 / 4 \mathrm{LEL}$ & $<4 \times 10^{4}$ & $>700$ \\
Preheated incineration & $<1500$ & $10^{4}-1.2 \times 10^{5}$ & $<45$ \\
Thermal storage incineration & $<1000$ & $<1.2 \times 10^{4}$ & $<45$ \\
Adsorption concentration & $10^{4}-10^{5}$ & $<10^{4}$ & $<150$ \\
Biodegradation & $<500$ & $<3 \times 10^{4}$ & $<80$ \\
Condensation & ${ }^{*}$ Lower explosive limit (LEL). &
\end{tabular}

In a survey of 771 industrial applications of VOCs elimination processes, including 330 cases in China and 441 cases in various other countries (Figure 8), Jinying $X_{i}$ et al. examined how often the various technologies were used [51]. The data showed that the most commonly used technology in China was adsorption (38\%), followed by catalytic combustion (22\%) and biodegradation (15\%), while in other countries, the most often utilized were biodegradation (29\%) and catalytic combustion (29\%), followed by adsorption (16\%). Due to its simple operation, low capital cost, and ability to recycle VOCs across a wide range of concentrations, adsorption was the most widely used technology in China. However, in some instances, the adsorption equipment was not well maintained and used correctly for recycling of VOCs. Adsorption, membrane purification, and condensation were the most effective methods, therefore most commonly applied, in instances where VOCs were present in concentrations above $10,000 \mathrm{mg} \cdot \mathrm{m}^{-3}$. Catalytic combustion and incineration were used for the destruction of VOCs in the concentration range of $2000 \sim 10,000 \mathrm{mg} \cdot \mathrm{m}^{-3}$, where recycling is not financially viable. Biodegradation and non-thermal plasma were applied for the treatment of VOCs in a lower concentration than $2000 \mathrm{mg} \cdot \mathrm{m}^{-3}$. Some examples are introduced in the following section to illustrate the application of these technologies for the elimination of pharmaceutical VOCs in China $[3,4,14,51,52]$.

In the production of cefuroxime axetil, cefuroxime sodium, and cefotaxime sodium, the emitted VOCs include methanol, acetone, dichloromethane, DMF, acetic ether, and cyclohexane. One reported setup for the removal of these VOCs via a combination of condensation and adsorption technologies is described in Figure 9. Firstly, a portion of the various solvents was removed across a three-stage condensing unit comprised of a single stage of recycled water condensation, followed by two stages of $7{ }^{\circ} \mathrm{C}$ water condensation. The VOCs which were unable to be removed via the condensation process were removed by a two-stage activated carbon adsorption tower. The VOC-rich waste gas feedstock had a total VOC concentration of $2400 \mathrm{mg} \cdot \mathrm{m}^{-3}$, of which $800 \mathrm{mg} \cdot \mathrm{m}^{-3}$ was attributed to 
methanol. It entered the first condensation unit at a rate of $2000 \mathrm{~m}^{3} \cdot \mathrm{h}^{-1}$. More than $95 \%$ of the total VOC content was removed after the combined condensation and adsorption process. As a result, the emitted concentration and discharge rate of methanol was reduced to $18.9-29.3 \mathrm{mg} \cdot \mathrm{m}^{-3}$ and $0.08 \mathrm{~kg} \cdot \mathrm{h}^{-1}$, respectively, while the concentration and discharge rate of the other VOCs present was $55-63 \mathrm{mg} \cdot \mathrm{m}^{-3}$ and $0.16 \mathrm{~kg} \cdot \mathrm{h}^{-1}$, respectively. Both of these levels were in accordance with the emission standards of Hebei province where the factory was located.

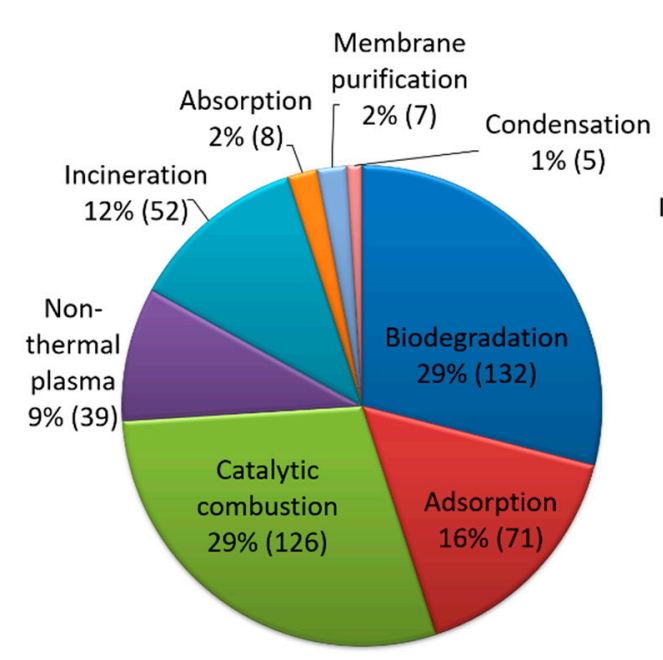

a

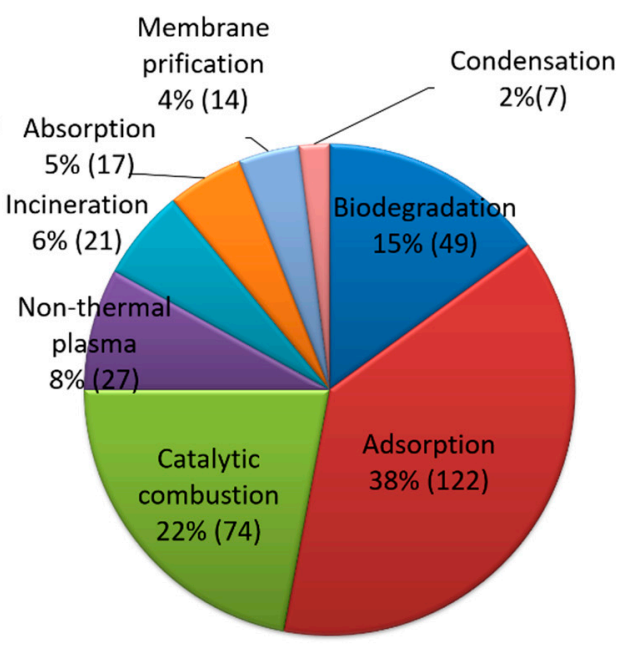

b

Figure 8. The ratios of VOCs elimination technologies used in China (a) and other countries (b) (the numbers in the brackets are the numbers of companies using the related technologies). Reprinted with permission from [51], copyright 2012, CNKI.

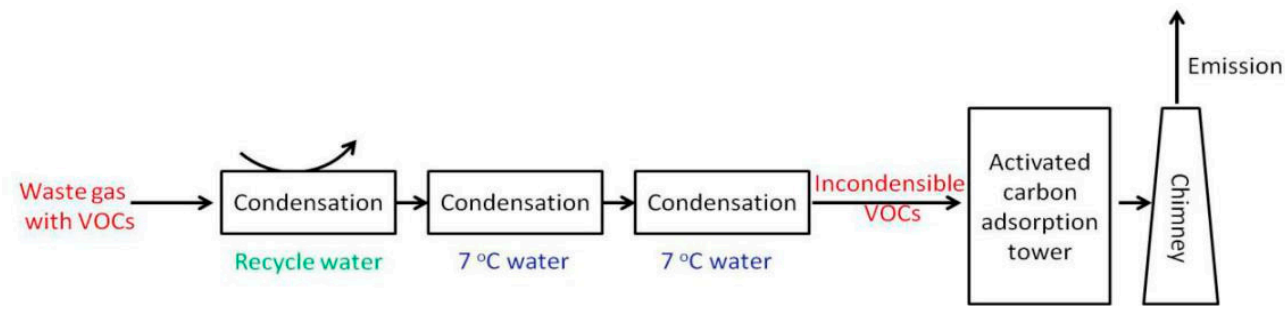

Figure 9. Representation of the VOC abatement process in a factory which produced cefuroxime axetil, cefuroxime sodium, and cefotaxime sodium. Reprinted with permission from [4], 2016, CNKI.

Yan Li has investigated the VOC abatement process in four separate companies in Taizhou city, Zhejiang province [4]. The main products in these four companies were clindamycin, clindamycin phosphate; losartan potassium, valsartan, nevirapine; meropenem, imipenem; and ciprofloxacin, spirolactone, respectively. There were three different procedures used in these four companies. The various procedures included adsorption, catalytic combustion, and non-thermal plasma technologies. The author assessed the efficiencies of the processes by measuring the concentration of a range of VOCs including benzene, toluene, xylene, methanol, formaldehyde, dichloromethane, chloroform, acetic ether, butylene oxide, acetonitrile, dimethylformamide, dysodia, and isopropanol before and after treatment. The results showed that after these elimination processes, the concentrations of VOCs in emitted waste gas were lower than the required concentration in standards for emissions of atmospheric pollutants from the pharmaceutical industry in Zhejiang province [53].

An example of a catalytic VOC treatment process is shown in Figure 10 below, which uses a regenerative catalytic oxidizer. The waste gas from the workshop was collected and combined with the waste gas from the sewage station, storehouse, and solid waste pile. The gas mixture was first washed with a water and alkali solution, then dehydrated and defogged. The dry gas was filtered, then heated 
with a preheater with an attached regenerative heat transfer. The preheated gas mixture entered the catalytic reactor and was combusted. After catalytic combustion, the gas was washed again with a water and alkali solution and emitted to high altitude atmosphere by a fan. The removal efficiencies of dysodia and non-methane hydrocarbons were $68.07 \%$ and $94.33 \%$, respectively. The running fee of the whole VOCs elimination system was about RMB 1 million per year.

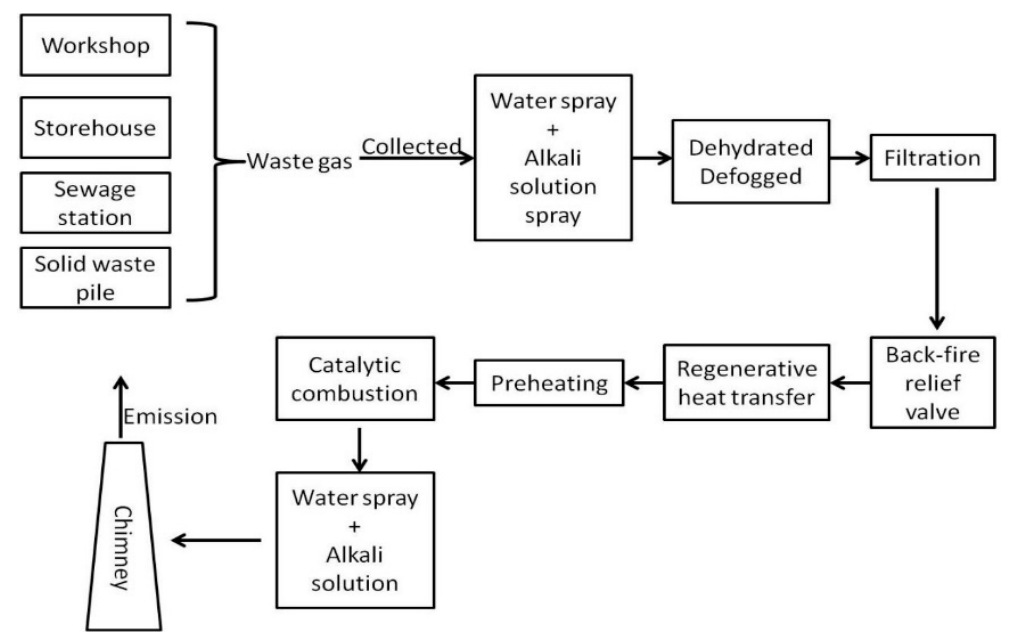

Figure 10. VOCs abatement process in the A factory. Reprinted with permission from [4], 2016, CNKI.

Two companies used a process which combines a regenerative thermal oxidizer (RTO) with three condensation stages connected in a series (Figure 11). The waste gas was pretreated in the workshop below determining the lower explosion limit (LEL) of the mixture. Then, fresh air was added in accordance with the determined LEL, such that the proper fuel, oxygen ratio, would be present in the RTO. After that, the gas mixture was oxidized in the RTO at a temperature of $850{ }^{\circ} \mathrm{C}$ with $98 \%$ of the thermal energy recycled. The high temperature gas exiting the RTO was cooled in a cooling tower, then further cooled across a three-stage condensation setup before it was emitted to the atmosphere. The dysodia concentration in the total vent was $300 \mathrm{mg} \cdot \mathrm{m}^{-3}$, while the concentration of non-methane hydrocarbons was lower than $85 \mathrm{mg} \cdot \mathrm{m}^{-3}$. These concentrations correspond to removal rates of $89 \%$ and $92 \%$ for dysodia and non-methane hydrocarbons, respectively. The main drawback of this procedure was that the condensation step was not effective for dichloromethane recycling and a lot of $\mathrm{HCl}$ generated from the incineration of chlorinated organic compounds, which in turn led to the corrosion of the equipment.

A process which combines the non-thermal plasma and catalytic oxidation techniques (oxidation of VOCs by $\mathrm{H}_{2} \mathrm{O}_{2}$ in a low $\mathrm{pH}$ ) to treat VOCs in waste gas is outlined in Figure 12. The waste gas, with a high VOC concentration, was pretreated, combined with exhaust gas, and washed with an alkali solution. Then, the washed gas entered the catalytic combustion/oxidation tower, where a portion of the VOCs content was oxidized to $\mathrm{CO}_{2}$ and $\mathrm{H}_{2} \mathrm{O}$. The oxidized waste gas was washed with water and entered a dehydrator to remove humidity. After that, the waste gas from the sewage station was added to the treated gas, upon which the gas mixture was treated with a non-thermal plasma, to remove additional VOCs. The gas was dehydrated again and entered into the second catalytic oxidation tower. The gas was washed by the alkali solution again and emitted to the atmosphere. The dysodia concentration was reduced by $84 \%$ to no more than $250 \mathrm{mg} \cdot \mathrm{m}^{-3}$. The removal efficiency of non-methane hydrocarbons content was approximately $92 \%$, with a concentration of less than $85 \mathrm{mg} \cdot \mathrm{m}^{-3}$ in the emitted gas.

These procedures (Figures 9-12 Figure 9 Figure 10 Figure 11 Figure 12) are representative of the range of VOCs elimination technologies currently in use in China. Almost all kinds of VOCs abatement technologies have been applied in the treatment of waste from the Chinese pharmaceutical industry. While the application of these technologies has successfully decreased the emission of VOCs, the 
high cost of investment and low efficiency are still the main factors which hinder the application of these technologies in the industry. The adsorption, absorption, and biodegradation technologies face challenges concerning the production of secondary pollutants, the desorption of adsorbed VOCs, and the production of waste water and sludge. The incineration process is effective in removing the issue of secondary waste production, but it requires a large energy input and has safety risks posed by the high temperature and use of a flame within a factory. The catalytic combustion and non-thermal plasma technologies partially circumvent the issues of waste and high temperature, but they are currently costly techniques with a short equipment lifetime. Thus, the continuing improvement of these technologies and development of new technologies is needed. With the new emission standards/law coming into effect, VOC elimination processes need to be upgraded in many Chinese pharmaceutical factories. As such, there is a desire for novel, effective, and energy efficient technologies in the near future.

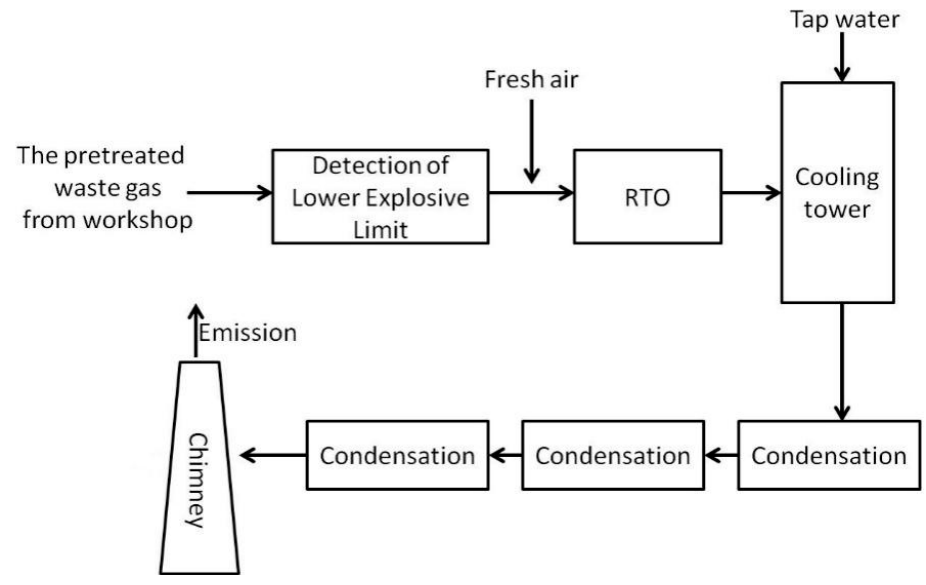

Figure 11. VOCs abatement process in the B and C factory, utilizing a regenerative thermal oxidizer (RTO) and condensation. Reprinted with permission from [4], 2016, CNKI.

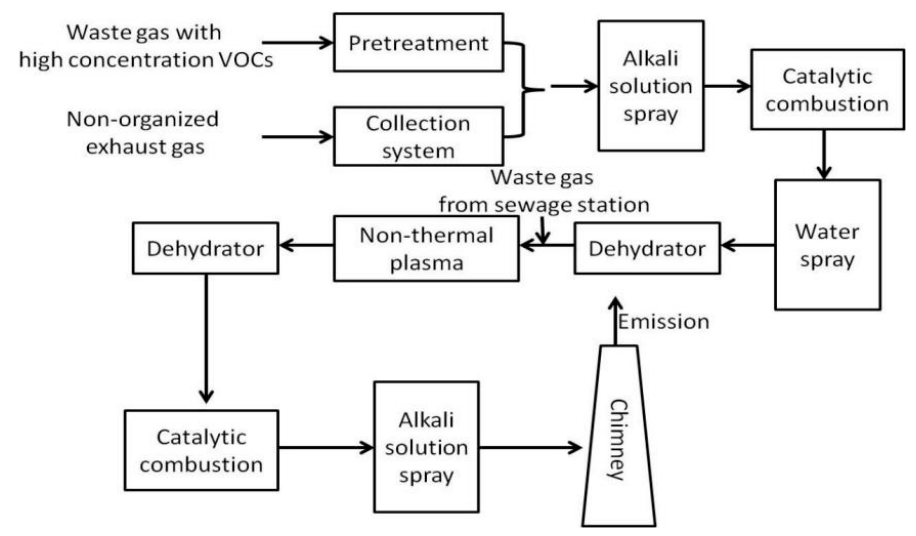

Figure 12. VOCs abatement process in the D factory. Reprinted with permission from [4], 2016, CNKI.

\section{The Developing Technologies for VOCs Elimination}

As outlined in the previous section, the VOC abatement technologies currently in use in China are hindered by some key limitations, such as high construction and running costs, low removal efficiencies for complex VOCs, and high temperature or energy requirements. To address these shortcomings, significant research is being carried out to improve the traditional methods of VOC removal, such as adsorption, catalytic combustion, and non-thermal plasma [1,2,33,41,43,44,47-49,54]. In addition, new technologies which can avoid the disadvantages of the traditional technologies have emerged, such as photocatalytic oxidation, condensation-oxidation, and electron beam treatment $[2,42,48,55]$. A summary of the various catalysts and conditions that have been investigated as active materials for the catalytic oxidation of VOCs is contained in Table 4 below. 
Table 4. Reported catalysts for VOCs elimination.

\begin{tabular}{|c|c|c|c|c|c|}
\hline VOCs & Refer & Catalyst & Catalytic Performance & Remarks & Catalytic Mechanism \\
\hline & {$[56]$} & $0.3 \% \mathrm{Pt} / \gamma-\mathrm{Al}_{2} \mathrm{O}_{3}$ & $\mathrm{~T}_{95}: 220^{\circ} \mathrm{C}$ & - & - \\
\hline & {$[57]$} & Pd-AuFeCeIM & $\mathrm{T}_{100}: 200{ }^{\circ} \mathrm{C}$ & - & - \\
\hline & {$[58]$} & $\begin{array}{c}\text { Layered copper } \\
\text { manganese oxide (LCMO) }\end{array}$ & $\begin{array}{l}\mathrm{T}_{50}: 218^{\circ} \mathrm{C} \\
\mathrm{T}_{90}: 240^{\circ} \mathrm{C}\end{array}$ & $\begin{array}{l}\text { The introduction of water vapor had } \\
\text { no significant effect on the catalyst. } \\
\text { The catalyst activity decreased about } \\
30 \% \text { after } \mathrm{SO}_{2} \text { was added. } \\
\text { The addition of } \mathrm{CO}_{2} \text { has no effect on } \\
\text { the catalytic activity of the catalyst. }\end{array}$ & $\begin{array}{l}\text { The larger surface area and the formation of } \\
\mathrm{Cu}^{2+}-\mathrm{O}^{2-}-\mathrm{Mn}^{4+} \text { entities at the interface } \\
\text { between } \mathrm{CuO} \text { and layered } \mathrm{MnO}_{2} \text { promoted } \\
\text { the } \mathrm{CO} \text { and VOCs oxidation. }\end{array}$ \\
\hline & [59] & $\mathrm{LaCoO}_{3}$ & $\mathrm{~T}_{50}: 330^{\circ} \mathrm{C}$ & $\begin{array}{c}\text { Water vapor has no significant effect } \\
\text { on the catalytic performance of } \\
\text { catalyst. }\end{array}$ & $\begin{array}{l}\text { Factors improve activity: (i) The larger } \\
\text { exposed surface, (ii) the composition of the } \\
\text { support provides increased oxygen mobility. }\end{array}$ \\
\hline & {$[60]$} & $\begin{array}{l}\mathrm{LaMnO}_{3} \\
\mathrm{LaCoO}_{3}\end{array}$ & $\begin{array}{l}\mathrm{T}_{50}: 301{ }^{\circ} \mathrm{C} \\
\mathrm{T}_{50}: 323^{\circ} \mathrm{C}\end{array}$ & - & - \\
\hline \multirow[t]{4}{*}{ Benzene } & {$[61]$} & $\gamma-\mathrm{MnO}_{2} / \mathrm{SmMnO}_{3}$ & $\begin{array}{l}\mathrm{T}_{50}: 213^{\circ} \mathrm{C} \\
\mathrm{T}_{90}: 226^{\circ} \mathrm{C}\end{array}$ & - & $\begin{array}{c}\text { Toluene-Mn ions }+ \text { surface reactive oxygen } \\
\rightarrow \text { benzaldehyde } \rightarrow \text { benzoic acid } \rightarrow \text { chain } \\
\text { carboxylic acids } \rightarrow \mathrm{CO}_{2} \text {. } \\
\text { Benzene } \rightarrow \text { (splitting of benzene, } \\
\text { hydrogenation, } \mathrm{H} \text { abstraction by OH }\end{array}$ \\
\hline & {$[63]$} & Mesoporous $\mathrm{TiO}_{2}$ & - & - & $\begin{array}{c}\text { radicals) six-member ring } \\
\text { cyclitols+aldehydes ketones etc. } \rightarrow \text { a class of } \\
\text { fulvene (isomerization) } \rightarrow \mathrm{CO}_{2}+\mathrm{H}_{2} \mathrm{O} \text {. } \\
\text { Benzene is mainly degraded by } \\
\text { photo-generated electron-hole pairs and } \\
\text { hydroxyl radicals. }\end{array}$ \\
\hline & {$[64]$} & Graphite- $\mathrm{SiO}_{2}-\mathrm{TiO}_{2}$ & & $\begin{array}{l}\text { The graphite- }-\mathrm{SiO}_{2}-\mathrm{TiO}_{2} \text { composites } \\
\text { exhibited higher photocatalytic } \\
\text { activity for degradation of benzene } \\
\text { gas under visible light irradiation } \\
\text { than that of pure } \mathrm{TiO}_{2} \text {. }\end{array}$ & $\begin{array}{l}\text { Optimum concentration of graphite } \\
\text { facilitates the separation of photogenerated } \\
\text { electron-hole pairs for graphite- } \mathrm{SiO}_{2}-\mathrm{TiO}_{2} \\
\text { composites by visible light. }\end{array}$ \\
\hline & {$[65]$} & $\mathrm{Pt} / \mathrm{Al}_{2} \mathrm{O}_{3}$ & - & - & - \\
\hline
\end{tabular}


Table 4. Cont.

\begin{tabular}{|c|c|c|c|c|c|}
\hline VOCs & Refer & Catalyst & Catalytic Performance & Remarks & Catalytic Mechanism \\
\hline \multirow{9}{*}{ Toluene } & {$[66]$} & $2.3 \mathrm{wt} \% \mathrm{Pt} / 3 \mathrm{DOM}-\mathrm{Mn}_{2} \mathrm{O}_{3}$ & $\begin{array}{l}\mathrm{T}_{50}: 165^{\circ} \mathrm{C} \\
\mathrm{T}_{90}: 194^{\circ} \mathrm{C}\end{array}$ & - & $\begin{array}{l}\text { A first-order reaction mechanism. Toluene } \\
\rightarrow \text { cat. Surface } \rightarrow \text { benzyl alcohol } \rightarrow \text { benzoic } \\
\text { acid and benzaldehyde } \rightarrow \text { (rise temperature }) \\
\text { maleic anhydride } \rightarrow \mathrm{CO}_{2} \text { and } \mathrm{H}_{2} \mathrm{O} \text {. }\end{array}$ \\
\hline & {$[67]$} & $\mathrm{Pd}\left(\right.$ shell)- $\mathrm{Au}$ (core) $/ \mathrm{TiO}_{2}$ & $\begin{array}{l}\mathrm{T}_{50}: 220^{\circ} \mathrm{C} \\
\mathrm{T}_{90}: 230^{\circ} \mathrm{C}\end{array}$ & - & Langmuir-Hinshelwood mechanism. \\
\hline & {$[68]$} & $\mathrm{Au}-\mathrm{Pd} / \mathrm{CeO}_{2}$ & $\begin{array}{l}\mathrm{T}_{50}: 120^{\circ} \mathrm{C} \\
\mathrm{T}_{90}: 150^{\circ} \mathrm{C}\end{array}$ & - & $\begin{array}{l}\text { Due to the synergistic effect between } \mathrm{Au} \text { and } \\
\mathrm{Pd} \text { nanoclusters, } \mathrm{Au}-\mathrm{Pd} / \mathrm{CeO}_{2} \text { bimetallic } \\
\text { catalysts are much better than } \mathrm{Au} \text { and } \mathrm{Pd} \\
\text { single metal catalysts. }\end{array}$ \\
\hline & [69] & $\mathrm{Mn}_{2} \mathrm{O}_{3}$ & $\begin{array}{l}\mathrm{T}_{50}: 231^{\circ} \mathrm{C} \\
\mathrm{T}_{90}: 239^{\circ} \mathrm{C}\end{array}$ & - & $\begin{array}{c}\text { Toluene }+ \text { cat. } \rightarrow \text { benzyl species }+ \text { active } \\
\text { oxygen species } \rightarrow \text { aromatic alkoxide } \rightarrow \\
\text { enzaldehyde } \rightarrow \text { benzoate species+active } \\
\text { oxygen specie } \rightarrow \text { maleic anhydrides } \rightarrow \mathrm{CO}_{2} \text {. }\end{array}$ \\
\hline & {$[58]$} & $\begin{array}{c}\text { Layered copper } \\
\text { manganese oxide (LCMO) }\end{array}$ & $\begin{array}{l}\mathrm{T}_{50}: 187^{\circ} \mathrm{C} \\
\mathrm{T}_{90}: 207^{\circ} \mathrm{C}\end{array}$ & - & - \\
\hline & [59] & $\mathrm{LaCoO}_{3}$ & $\mathrm{~T}_{50}: 244^{\circ} \mathrm{C}$ & - & - \\
\hline & {$[70]$} & $\mathrm{La}_{0.8} \mathrm{Sr}_{0.2} \mathrm{CoO}_{3}$ & $\begin{array}{c}\mathrm{T}_{50}:<160^{\circ} \mathrm{C} \\
\mathrm{T}_{99}: 300^{\circ} \mathrm{C}\end{array}$ & - & $\begin{array}{c}\text { Partial substitution of strontium }(\mathrm{Sr}) \text { for } \\
\text { lanthanum }(\mathrm{La}) \text { greatly increased the oxygen } \\
\text { vacancies in the surface regions to enhance } \\
\text { catalytic activity. }\end{array}$ \\
\hline & {$[71]$} & $\begin{array}{l}\mathrm{SrTi}_{1-\chi} \mathrm{Cu}_{X} \mathrm{O}_{3} \\
\mathrm{SrTi}_{1-\chi} \mathrm{Mn}_{X} \mathrm{O}_{3}\end{array}$ & $\begin{array}{l}\mathrm{T}_{50}: 343^{\circ} \mathrm{C} \\
\mathrm{T}_{90}: 398{ }^{\circ} \mathrm{C} \\
\mathrm{T}_{50}: 302^{\circ} \mathrm{C} \\
\mathrm{T}_{90}: 335^{\circ} \mathrm{C} \\
\mathrm{T}_{50}: 250^{\circ} \mathrm{C}\end{array}$ & - & $\begin{array}{l}\text { Incorporation of Mn attributed a higher } \\
\text { amount of oxygen vacancies in the } \\
\text { perovskite surface to promote the toluene } \\
\text { conversion. Supra facial mechanism. }\end{array}$ \\
\hline & {$[72]$} & $\begin{array}{c}\mathrm{LaMn}_{1-\mathrm{x}} \mathrm{B}_{\mathrm{x}} \mathrm{O}_{3}(\mathrm{~B}=\mathrm{Co}, \mathrm{Ni} \\
\mathrm{Cu}, \mathrm{Al})\end{array}$ & $\begin{array}{c}234^{\circ} \mathrm{C} \\
226^{\circ} \mathrm{C} \\
242^{\circ} \mathrm{C} \\
\mathrm{T}_{90}: \text { none } \\
278^{\circ} \mathrm{C} \\
271^{\circ} \mathrm{C} \\
318^{\circ} \mathrm{C}\end{array}$ & - & $\begin{array}{l}\text { Addition of nickel into } \mathrm{LaMnO}_{3} \text { can improve } \\
\text { the catalytic oxidation of toluene by the } \\
\text { generation of more } \mathrm{Mn}^{4+} \text { species and } \\
\text { oxygen vacancies and the enhancing } \\
\text { reducibility at a low temperature. }\end{array}$ \\
\hline
\end{tabular}


Table 4. Cont.

\begin{tabular}{|c|c|c|c|c|c|}
\hline VOCs & Refer & Catalyst & Catalytic Performance & Remarks & Catalytic Mechanism \\
\hline & {$[61]$} & $\gamma-\mathrm{MnO} 2 / \mathrm{SmMnO}_{3}$ & $\begin{array}{l}\mathrm{T}_{50}: 187^{\circ} \mathrm{C} \\
\mathrm{T}_{90}: 208^{\circ} \mathrm{C}\end{array}$ & - & - \\
\hline & [73] & $\mathrm{LaMnO} 3 / \delta-\mathrm{MnO} 2$ & $\mathrm{~T}_{90}: 258^{\circ} \mathrm{C}$ & - & - \\
\hline & [74] & $\mathrm{LaMnO}_{3}$ & $\begin{array}{l}\mathrm{T}_{50}: 258^{\circ} \mathrm{C} \\
\mathrm{T}_{90}: 275^{\circ} \mathrm{C}\end{array}$ & - & - \\
\hline & [75] & $\begin{array}{c}\mathrm{MnO}_{\mathrm{x}} / \mathrm{H}-\text { Beta-SDS } \\
\mathrm{MnO}_{\mathrm{x}} / \mathrm{K}-\text { Beta-SDS } \\
\mathrm{MnOx} / \text { Si-Beta }\end{array}$ & $\begin{array}{l}\mathrm{T}_{50}: 253,262,280^{\circ} \mathrm{C} \\
\mathrm{T}_{90}: 285,295,312^{\circ} \mathrm{C}\end{array}$ & - & $\begin{array}{l}\text { Mars-Van Krevelen mechanism. Organic } \\
\text { molecules }+ \text { lattice oxygen } \rightarrow \text { oxygen vacancy } \\
+\mathrm{CO}_{2}+\mathrm{H}_{2} \mathrm{O} \text {, oxygen vacancy }+\mathrm{O}_{2} \rightarrow \text { lattice } \\
\text { oxygen. }\end{array}$ \\
\hline & [76] & $\mathrm{Mn} / \mathrm{R}-\mathrm{SBA}-15$ & $\mathrm{~T}_{98}: 240^{\circ} \mathrm{C}$ & - & $\begin{array}{l}\text { MVK mechanism. } \mathrm{Mn}_{2} \mathrm{O}_{3}-\mathrm{MnO}_{2} / \mathrm{R}-\mathrm{SBA}-15 \\
\text { supply more lattice oxygen species. }\end{array}$ \\
\hline & [77] & $\mathrm{LaFeO}_{3} /$ black-TiO 2 & $\begin{array}{c}\text { The removal efficiency } \\
\text { of black } \mathrm{TiO}_{2} \text { and } \\
\mathrm{LaFeO}_{3} \text { for toluene was } \\
89 \% \text { and } 98 \% \text {, } \\
\text { respectively, and the } \\
\text { removal efficiency for } \\
\text { IPA was } 90 \% \text { and } 94 \% \text {, } \\
\text { respectively. }\end{array}$ & - & $\begin{array}{c}\text { Cat. }+ \text { photon (the wavelength shorter than } \\
440 \mathrm{~nm} \text { ) } \rightarrow \text { electrons }+\mathrm{O}_{2} \rightarrow \mathrm{O}^{2-} \\
\mathrm{O}^{2-}+\text { toluene and IPA } \rightarrow \mathrm{CO}_{2}+\mathrm{H}_{2} \mathrm{O}_{(\mathrm{g})}\end{array}$ \\
\hline & [78] & $\mathrm{Pd} / \mathrm{CeO}_{2} / \gamma-\mathrm{Al}_{2} \mathrm{O}_{3}$ & $\mathrm{~T}_{98}: 205^{\circ} \mathrm{C}$ & - & - \\
\hline & [80] & $\begin{array}{l}\mathrm{CuO} / \mathrm{ZnO} \text { Nanocomposite } \\
\text { photocatalysts }\end{array}$ & - & $\begin{array}{l}\text { Under the condition of } 3 \% \mathrm{Fe} \\
\text { loading, the oxidation of toluene is } \\
\text { the best one. }\end{array}$ & $\begin{array}{l}\text { Photooxidative activity and stability over } \\
\mathrm{ZnO} \text { are improved by loading } \mathrm{CuO} \text {. } \\
\text { (1) Direct removal caused by the collision of } \\
\text { electrons or oxidation caused by the } \\
\text { gas-phase radicals }\left(\mathrm{O} \bullet, \mathrm{OH} \bullet, \mathrm{N}_{2} \bullet, \mathrm{NO} \bullet \text {, }\right. \\
\mathrm{NO}_{2} \bullet \text { ) in gas phase; (2) the reaction between } \\
\text { adsorbed toluene or other intermediates and } \\
\text { the active species }(\mathrm{O} \bullet, \mathrm{OH} \bullet \text { ) on the catalyst } \\
\text { surface. } \mathrm{O}_{2} \text { might be fixed on the catalyst } \\
\text { surface via facile interconversion between } \\
\mathrm{Fe}^{2+} \text { and } \mathrm{Fe}^{3+} \text { states and then be transported } \\
\text { to the toluene or intermediates leading to } \\
\qquad \mathrm{CO}_{2} \text { formation. }\end{array}$ \\
\hline
\end{tabular}


Table 4. Cont.

\begin{tabular}{|c|c|c|c|c|c|}
\hline VOCs & Refer & Catalyst & Catalytic Performance & Remarks & Catalytic Mechanism \\
\hline Butanol & [56] & $0.3 \% \mathrm{Pt} / \gamma-\mathrm{Al}_{2} \mathrm{O}_{3}$ & $\mathrm{~T}_{95}: 200^{\circ} \mathrm{C}$ & - & - \\
\hline \multirow[t]{2}{*}{$\mathrm{CH}_{3} \mathrm{SSCH}_{3}$} & [81] & $\mathrm{Pt}-\mathrm{Au} / \mathrm{Ce}-\mathrm{Al}$ & $\begin{array}{l}\mathrm{T}_{50}: 425^{\circ} \mathrm{C} \\
\mathrm{T}_{90}: 480^{\circ} \mathrm{C}\end{array}$ & - & $\begin{array}{l}\text { The addition of Au improved the selectivity } \\
\text { of ceria-containing catalysts by decreasing } \\
\text { the formation of byproducts. This may have } \\
\text { had a connection to a lower amount of } \\
\text { reactive oxygen after the Au addition. }\end{array}$ \\
\hline & [81] & $\mathrm{Cu}-\mathrm{Au} / \mathrm{Ce}-\mathrm{Al}$ & $\begin{array}{l}\mathrm{T}_{50}: 275^{\circ} \mathrm{C} \\
\mathrm{T}_{90}: 370^{\circ} \mathrm{C}\end{array}$ & - & - \\
\hline Chlorobenzene & [82] & $\mathrm{Pt} / \mathrm{CrOOH}$ & $\begin{array}{l}\mathrm{T}_{50}: 340^{\circ} \mathrm{C} \\
\mathrm{T}_{90}: 378^{\circ} \mathrm{C}\end{array}$ & - & $\begin{array}{l}\text { Platinum accelerates the hydrolysis of } \mathrm{Cr}-\mathrm{Cl} \\
\text { bonds formed at the } \mathrm{CrOOH} \text { surface and } \\
\text { determines the catalyst stability. }\end{array}$ \\
\hline Dichloroethane & [82] & $\mathrm{Pt} / \mathrm{CrOOH}$ & $\begin{array}{l}\mathrm{T}_{50}: 283^{\circ} \mathrm{C} \\
\mathrm{T}_{90}: 317^{\circ} \mathrm{C}\end{array}$ & - & - \\
\hline Dichloromethane & [83] & $\mathrm{CeO}_{2}$ & $\begin{array}{l}\mathrm{T}_{90}: 260^{\circ} \mathrm{C} \\
\mathrm{T}_{90}: 160^{\circ} \mathrm{C}\end{array}$ & $\begin{array}{l}\text { The addition of } 3 \%(\mathrm{v} / \mathrm{v}) \text { water can } \\
\text { obviously inhibit the catalytic } \\
\text { decomposition of VOCs on } \mathrm{CeO}_{2} \text {. }\end{array}$ & $\begin{array}{l}\text { Trichloroethylene }+\mathrm{CeO}_{2} \rightarrow \mathrm{C}_{2} \mathrm{HCl} \rightarrow \mathrm{HCl} \text {, } \\
\qquad \mathrm{Cl}_{2}, \mathrm{CO}_{2} \text { and trace } \mathrm{CO}\end{array}$ \\
\hline Dichlorobenzene & [84] & H-ZSM-5 and Na-ZSM-5 & - & - & - \\
\hline $\begin{array}{l}\text { Trichloro } \\
\text { benzene }\end{array}$ & {$[84]$} & H-ZSM-5 and Na-ZSM-5 & - & - & - \\
\hline DCE & {$[85]$} & $\mathrm{CeO}_{2} @ \mathrm{SiO}_{2}-400$ & $\begin{array}{l}\mathrm{T}_{50}: 219^{\circ} \mathrm{C} \\
\mathrm{T}_{90}: 275^{\circ} \mathrm{C}\end{array}$ & $\begin{array}{c}\text { The conversion of } 1 \text { vol } \% \mathrm{H}_{2} \mathrm{O} \text { and } 3 \\
\text { vol } \% \mathrm{H}_{2} \mathrm{O} \text { decreased by } 6 \% \text { and } \\
19 \% \text {, respectively. }\end{array}$ & $\begin{array}{l}\mathrm{DCE}+\text { acid sites } \rightarrow \mathrm{HCl}+\mathrm{VC} \text {. (1) } \mathrm{VC} \rightarrow \text { reactive } \\
\text { carbonium ion } \rightarrow \text { adsorbed alcohol } \\
\text { species } \rightarrow \text { acetate species. Or (2) } \\
\text { VC } \rightarrow \text { 1,1,2-trichloroethane } \rightarrow \text { dichloroethylene } \\
\text { formed (subsequent chlorination reactions) } \\
\quad \rightarrow \mathrm{H}_{2} \mathrm{O}, \mathrm{CO}_{x} \text { and } \mathrm{HCl} \text {. }\end{array}$ \\
\hline Ethylbenzene & [61] & $\gamma-\mathrm{MnO}_{2} / \mathrm{SmMnO}_{3}$ & $\begin{array}{l}\mathrm{T}_{50}: 201^{\circ} \mathrm{C} \\
\mathrm{T}_{90}: 217^{\circ} \mathrm{C}\end{array}$ & - & - \\
\hline
\end{tabular}


Table 4. Cont.

\begin{tabular}{|c|c|c|c|c|c|}
\hline VOCs & Refer & Catalyst & Catalytic Performance & Remarks & Catalytic Mechanism \\
\hline Formaldehyde & [86] & $\mathrm{TiO}_{2}$ & $\begin{array}{l}\mathrm{TiO}_{2} \text { degraded almost } \\
100 \% \text { of formaldehyde } \\
\text { or acetaldehyde at a } \\
\text { starting concentration of } \\
400-500 \text { ppb with a } \\
\text { relative humidity of } \\
40 \% \text {. }\end{array}$ & - & $\begin{array}{l}\text { The rate-determining step is the adsorption } \\
\text { (external diffusion) on the catalysts active } \\
\text { sites, thus the higher surface area, the higher } \\
\text { the degradation. }\end{array}$ \\
\hline Acetaldehyde & [86] & $\mathrm{TiO}_{2}$ & - & - & - \\
\hline \multirow{2}{*}{ Acetone } & [78] & $\mathrm{Pd} / \mathrm{CeO}_{2} / \gamma-\mathrm{Al}_{2} \mathrm{O}_{3}$ & $\mathrm{~T}_{98}: 220^{\circ} \mathrm{C}$ & - & - \\
\hline & [87] & $\mathrm{CuO} / \mathrm{g}-\mathrm{Al}_{2} \mathrm{O}_{3}$ & so $-2+2$ & $\begin{array}{c}5.0 \mathrm{wt} \% \mathrm{CuO} / \mathrm{g}-\mathrm{Al}_{2} \mathrm{O}_{3} \text { catalyst has } \\
\text { the highest removal rate of acetone, } \\
\text { reaching } 67.9 \% .\end{array}$ & $\begin{array}{l}\text { Both short-lived radicals and } \\
\text { acetone/intermediates can be adsorbed on } \\
\text { the catalyst surfaces to initiate a series of } \\
\text { surface oxidation reactions, forming } \mathrm{CO} \\
\mathrm{CO}_{2}, \mathrm{H}_{2} \mathrm{O} \text {, and byproducts. }\end{array}$ \\
\hline Ethyl acetate & [78] & $\mathrm{Pd} / \mathrm{CeO}_{2} / \gamma-\mathrm{Al}_{2} \mathrm{O}_{3}$ & $\mathrm{~T}_{98}: 275^{\circ} \mathrm{C}$ & - & - \\
\hline Benzoquinone & [88] & $\mathrm{g}-\mathrm{C}_{3} \mathrm{~N}_{4}$ & - & - & $\begin{array}{l}\mathrm{OH} \\
\text { radicals+phenol } \rightarrow \text { dihydroxycyclohexadienyl } \\
\text { radical adducts } \rightarrow \text { phenoxy radicals } \rightarrow \mathrm{H}_{2} \mathrm{O}(\mathrm{a} \\
\text { very slow process), adducts+dissolved } \\
\mathrm{O}_{2} \rightarrow \text { dihydroxy intermediates }\left(-\mathrm{HO}_{2}\right) \\
\rightarrow \mathrm{CO}_{2}+\mathrm{H}_{2} \mathrm{O} .\end{array}$ \\
\hline $\begin{array}{l}\text { Hydroquinone } \\
\text { Catechol }\end{array}$ & [88] & $\mathrm{g}-\mathrm{C}_{3} \mathrm{~N}_{4}$ & - & - & - \\
\hline
\end{tabular}


Table 4. Cont.

\begin{tabular}{|c|c|c|c|c|c|}
\hline VOCs & Refer & Catalyst & Catalytic Performance & Remarks & Catalytic Mechanism \\
\hline \multirow[t]{3}{*}{ O-xylene } & [89] & $\mathrm{Ag} / \mathrm{NiO}_{\mathrm{x}}-\mathrm{MnO}_{2}$ & $\begin{array}{l}\mathrm{T}_{50}: 145^{\circ} \mathrm{C} \\
\mathrm{T}_{90}: 190^{\circ} \mathrm{C}\end{array}$ & $\begin{array}{l}\text { The catalytic activity decreased with } \\
\text { the addition of water vapor, but } \\
\text { recovered after the removal of water } \\
\text { vapor. }\end{array}$ & $\begin{array}{c}\text { Mars-Van Krevelen mechanism. } \\
\text { (i) Electrophilic } \mathrm{O}_{1}+\text { aromatic ring } \rightarrow \text { maleate; } \\
\text { (ii) } \mathrm{O}_{2}+\text { Cat. } \rightarrow \text { electrophilic oxygen species } \\
\left.\mathrm{O}_{2}{ }^{2-}, \mathrm{O}^{2-}, \mathrm{O}^{-}\right) \text {, electrophilic oxygen } \\
\text { species+aromatic ring } \rightarrow \text { maleate, } \\
\text { carbonate }+\left(\mathrm{O}_{2} \cdot \mathrm{O}_{2}^{2-}, \mathrm{O}^{2-}, \mathrm{O}^{-} \text {or } \mathrm{O}_{2}\right) \\
+\mathrm{Ag} \rightarrow \text { nucleophilic oxygen, nucleophilic } \\
\text { oxygen + maleate and carboxylates } \rightarrow \mathrm{CO}_{2} \\
\text { and } \mathrm{H}_{2} \mathrm{O} \text {. }\end{array}$ \\
\hline & [58] & $\begin{array}{c}\text { Layered copper } \\
\text { manganese oxide (LCMO) }\end{array}$ & $\begin{array}{l}\mathrm{T}_{50}: 213^{\circ} \mathrm{C} \\
\mathrm{T}_{90}: 227^{\circ} \mathrm{C}\end{array}$ & - & - \\
\hline & {$[61]$} & $\gamma-\mathrm{MnO} 2 / \mathrm{SmMnO}_{3}$ & $\begin{array}{l}\mathrm{T}_{50}: 232{ }^{\circ} \mathrm{C} \\
\mathrm{T}_{90}: 250^{\circ} \mathrm{C}\end{array}$ & - & - \\
\hline O-dichlorobenzene & [90] & CeMn30 & $\begin{array}{c}\text { GHSV }=15,000 \mathrm{~mL} /(\mathrm{g} \cdot \mathrm{h}) \\
\mathrm{T}_{50}: 291^{\circ} \mathrm{C} \\
\mathrm{T}_{90}: 347^{\circ} \mathrm{C} \\
\text { GHSV }=7500 \mathrm{~mL} /(\mathrm{g} \cdot \mathrm{h}) \\
\mathrm{T}_{90}: 224^{\circ} \mathrm{C} \\
\text { GHSV }=30,000 \mathrm{~mL} /(\mathrm{g} \cdot \mathrm{h}) . \\
\mathrm{T}_{90}: 360^{\circ} \mathrm{C}\end{array}$ & $\begin{array}{l}\text { The addition of low concentration of } \\
\text { water vapor is beneficial to the early } \\
\text { reaction, but will weaken the } \\
\text { catalytic activity of catalyst. }\end{array}$ & $\begin{array}{l}\text { O-dichlorobenzene molecules+basic oxygen } \\
(\mathrm{Mn}-\mathrm{O}-\mathrm{Ce}-\mathrm{V} \ddot{\mathrm{O}} \text {, nucleophilic } \\
\text { substitution }) \rightarrow \mathrm{HCl}-\mathrm{H}-\mathrm{O}-\mathrm{Ce}-\mathrm{V} \ddot{\mathrm{o}}+\text { phenyl. } \\
\text { Mn-O-Ce weakened } \mathrm{HCl}-\mathrm{H}-\mathrm{O}-\mathrm{Ce}-\mathrm{Vö} \text { and } \\
\text { then prevented Deacon reaction and chlorine } \\
\text { poisoning. Phenyl+ active lattice } \\
\text { oxygen } \rightarrow \text { organic intermediates } \rightarrow \mathrm{CO}_{2}+\mathrm{H}_{2} \mathrm{O} \text {. } \\
\text { (Mars-Van Krevelen mechanism). }\end{array}$ \\
\hline $\mathrm{CO}$ & [58] & $\begin{array}{c}\text { Layered copper } \\
\text { manganese oxide (LCMO) }\end{array}$ & $\begin{array}{l}\mathrm{T}_{50}: 62{ }^{\circ} \mathrm{C} \\
\mathrm{T}_{90}: 76^{\circ} \mathrm{C}\end{array}$ & - & - \\
\hline Cyclohexane & [70] & $\mathrm{La}_{0.8} \mathrm{Sr}_{0.2} \mathrm{CoO}_{3}$ & $\begin{array}{l}\mathrm{T}_{50}: 180^{\circ} \mathrm{C} \\
\mathrm{T}_{99}: 260^{\circ} \mathrm{C}\end{array}$ & - & - \\
\hline $\mathrm{CH}_{3} \mathrm{SH}$ & [91] & $\mathrm{La}(13) / \mathrm{HZSM}-5$ & $\mathrm{~T}_{100}: 40^{\circ} \mathrm{C}$ & - & $\begin{array}{l}\text { The La-modified HZSM- } 5 \text { increased basic } \\
\text { sites and displayed better adsorption ability } \\
\text { to } \mathrm{CH}_{3} \mathrm{SH} \text {, decreased in strong acid sites and } \\
\text { suppressed the formation of coke deposit. }\end{array}$ \\
\hline
\end{tabular}


Table 4. Cont.

\begin{tabular}{|c|c|c|c|c|c|}
\hline VOCs & Refer & Catalyst & Catalytic Performance & Remarks & Catalytic Mechanism \\
\hline $\mathrm{C}_{2} \mathrm{H}_{3} \mathrm{CN}$ & [92] & $\begin{array}{c}\mathrm{Cu}-\mathrm{ZSM}-5\left(\mathrm{SiO}_{2} / \mathrm{Al}_{2} \mathrm{O}_{3}=\right. \\
26)\end{array}$ & $\mathrm{T}_{90}: 325^{\circ} \mathrm{C}$ & $\begin{array}{l}\text { The catalyst has good resistance to } \\
\text { steam poisoning. }\end{array}$ & $\begin{array}{c}\text { Without } \mathrm{H}_{2} \mathrm{O}: \\
\mathrm{C}_{2} \mathrm{H}_{3} \mathrm{CN}-\mathrm{SCC}+\mathrm{O}_{2} \rightarrow \mathrm{NCO} \rightarrow \text { oxidation } \\
\text { products; with } \mathrm{H}_{2} \mathrm{O}: \mathrm{C}_{2} \mathrm{H}_{3} \mathrm{CN}-\mathrm{SCC}+\mathrm{O}_{2}+ \\
\mathrm{H}_{2} \mathrm{O} \rightarrow \mathrm{NH}_{3} \rightarrow \text { products. }\end{array}$ \\
\hline Cumene & [84] & H-ZSM-5 and Na-ZSM-5 & - & - & - \\
\hline \multirow{3}{*}{ Isopropanol } & [60] & $\begin{array}{l}\mathrm{LaMnO}_{3} \\
\mathrm{LaCoO}_{3}\end{array}$ & $\begin{array}{l}\mathrm{T}_{50}: 216^{\circ} \mathrm{C} \\
237^{\circ} \mathrm{C}\end{array}$ & - & - \\
\hline & [79] & $\begin{array}{l}\mathrm{CuO} / \mathrm{ZnO} \text { Nanocomposite } \\
\text { photocatalysts }\end{array}$ & - & - & $\begin{array}{l}\text { Photooxidative activity and stability over } \\
\mathrm{ZnO} \text { are improved by loading } \mathrm{CuO} \text {. }\end{array}$ \\
\hline & [93] & $\mathrm{Au}-\mathrm{Ag} / \mathrm{CeO}_{2}$ & $\begin{array}{l}\mathrm{T}_{50}: 105^{\circ} \mathrm{C} \\
\mathrm{T}_{90}: 158^{\circ} \mathrm{C} \\
\end{array}$ & - & MVK redox mechanism. \\
\hline \multirow{2}{*}{$\begin{array}{l}\text { Propyl } \\
\text { alcohol }\end{array}$} & {$[60]$} & $\begin{array}{l}\mathrm{LaMnO}_{3} \\
\mathrm{LaCoO}_{3}\end{array}$ & $\begin{array}{l}\mathrm{T}_{50}: 203^{\circ} \mathrm{C} \\
222{ }^{\circ} \mathrm{C}\end{array}$ & - & - \\
\hline & [70] & $\mathrm{La}_{0.8} \mathrm{Sr}_{0.2} \mathrm{CoO}_{3}$ & $\mathrm{~T}_{90}: 160^{\circ} \mathrm{C}$ & - & - \\
\hline Propane & [94] & $\mathrm{LaCoO}_{3}$ & $\begin{array}{l}\mathrm{T}_{50}: 208^{\circ} \mathrm{C} \\
\mathrm{T}_{90}: 238^{\circ} \mathrm{C}\end{array}$ & - & MVK mechanism. \\
\hline Methane & [95] & Nanocubic $\mathrm{MnO}_{2}$ & $\begin{array}{l}\mathrm{T}_{50}: 293^{\circ} \mathrm{C} \\
\mathrm{T}_{90}: 350^{\circ} \mathrm{C}\end{array}$ & - & $\begin{array}{c}\mathrm{CH}_{4}+\text { lattice oxygen or surface oxygen } \\
\text { vacancies }\left(\mathrm{MnO}_{2}-\mathrm{C}\right) \rightarrow \text { carboxylate species } \\
\text { (Langmuir-Hinshelwood route) }+ \text { active } \\
\text { oxygen species } \rightarrow \mathrm{CO}_{2} \text { and } \mathrm{H}_{2} \mathrm{O} \text {. }\end{array}$ \\
\hline
\end{tabular}


Table 4. Cont.

\begin{tabular}{|c|c|c|c|c|c|}
\hline VOCs & Refer & Catalyst & Catalytic Performance & Remarks & Catalytic Mechanism \\
\hline \multirow{6}{*}{ VOCs } & {$[96]$} & $\mathrm{MnO}_{2}$ & $\begin{array}{l}\mathrm{T}_{50}: 233^{\circ} \mathrm{C} \\
\mathrm{T}_{90}: 256^{\circ} \mathrm{C}\end{array}$ & - & Toluene + Mn cations $\rightarrow \mathrm{CO}_{2}$ and $\mathrm{H}_{2} \mathrm{O}$. \\
\hline & [97] & $\mathrm{LaMnO}_{3}$ and $\mathrm{LaCoO}_{3}$ & $\begin{array}{c}290 . \\
-\end{array}$ & - & - \\
\hline & [98] & $\mathrm{La}^{3+}-\mathrm{TiO}_{2}$ and $\mathrm{Nd}^{3+}-\mathrm{TiO}_{2}$ & $\begin{array}{l}1.2 \% \mathrm{La}^{3+}-\mathrm{TiO}_{2} \text { had the } \\
\text { highest photocatalytic } \\
\text { activity. }\end{array}$ & - & - \\
\hline & [99] & $\mathrm{TiO}_{2} / \mathrm{Pd}$ & $\begin{array}{l}\text { The conversion rate of } \\
\text { VOCs reached } 90 \% \\
\text { when the residence time } \\
\text { was } 27 \mathrm{~s} \text {. }\end{array}$ & - & - \\
\hline & {$[100]$} & $\mathrm{MesoTiO}_{2}$ /hydro-CF & 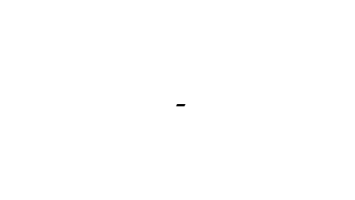 & - & $\begin{array}{l}\text { Promotion effects on degradation of gaseous } \\
\text { polar acetone come from well crystallized } \\
\text { anatase nanocrystals, hydro-CF skeleton for } \\
\text { adsorption, and fast mass transportation } \\
\text { within the hierarchical frameworks. }\end{array}$ \\
\hline & [101] & $\mathrm{CsX}, \mathrm{NaX}$, and $\mathrm{HY}$ & $\mathrm{T}_{100}: 200{ }^{\circ} \mathrm{C}$ & - & $\begin{array}{c}\text { Mars-Van Krevelen mechanism, involving } \\
\text { several redox steps. }\end{array}$ \\
\hline \multirow[t]{2}{*}{$\mathrm{O}_{3}$} & [102] & Mn/ZSM-5 & $\begin{array}{l}\mathrm{O}_{3} \text { can be efficiently } \\
\text { decomposed by the } \\
\text { Mn/ZSM- } 5 \text { and used for } \\
\text { benzene degradation } \\
\text { through the OZCO. }\end{array}$ & - & $\begin{array}{c}\text { Benzene }+\bullet \mathrm{OH} \rightarrow \text { phenol } \rightarrow \text { benzoquinone } \rightarrow \mathrm{CO}_{2} \\
+\mathrm{H}_{2} \mathrm{O} .\end{array}$ \\
\hline & [103] & $\mathrm{CoMnO}_{x} / \mathrm{TiO}_{2}$ & - & $\begin{array}{l}\text { When the temperature is } 320^{\circ} \mathrm{C} \text {, the } \\
\text { decomposition efficiency of } \mathrm{O}_{3} \text { is } \\
98 \% \text {. }\end{array}$ & - \\
\hline Vinyl chloride & [104] & $\begin{array}{l}\mathrm{HCl} \text { modified } \\
\mathrm{La}_{0.5} \mathrm{Sr}_{0.5} \mathrm{MnO}_{3}\end{array}$ & $\mathrm{~T}_{100}: 300^{\circ} \mathrm{C}$ & - & $\begin{array}{c}\text { Doping and acid treatment obviously } \\
\text { promote the active } \mathrm{Mn}^{4+} \text { species amount } \\
\text { and oxygen activation ability, and affect the } \\
\text { chloric by-product distribution. Lower } \\
\text { temperature inhibits the Deacon reaction } \\
\text { and chlorination. }\end{array}$ \\
\hline
\end{tabular}




\subsection{Catalytic Combustion}

Catalytic combustion has received attention recently, as it shows great potential to address the shortcomings of the incineration method. Catalytic combustion is suitable for waste gas streams with low VOC concentrations and a moderate flow rate. Compared with the incineration method, catalytic combustion has been shown to efficiently remove VOCs from waste streams with a wide range of VOC concentrations. In addition, it operates at a lower temperature ( 293 versus $673 \mathrm{~K}$ ) than incineration and resistant to the production of undesirable byproducts, such as dioxins and $\mathrm{NO}_{\mathrm{x}}$ [105]. The diversity of VOC species necessitates the development of different kinds of catalysts for the combustion method, with commonly used catalysts including noble metals, non-noble metal oxides concentrated oxidation catalysts $[1,2,41-44]$.

Previous reviews covering certain aspects of the catalytic combustion of VOCs have been published. For example, K. Everaert et al. reviewed, analyzed, and discussed the reaction kinetics, reactors, and reaction conditions of catalytic combustion research prior to 2004 [41]. Muhammad Shahzad Kamal et al. and Zhixiang Zhang et al. covered the recent progress in the development of combustion catalysts [2,44]. L.F. Liotta and W.B. Li et al. reviewed the mechanism of VOCs catalytic combustion over noble metal catalysts and non-noble metal catalysts, respectively $[1,42]$. In this section, we will briefly introduce the more recent development of new catalysts materials which have been used in this process, analyze the advantages and disadvantages they provide, and strategies for their successful implementation.

\subsubsection{Noble Metal Catalysts}

The general consensus of previous studies is that noble metal catalysts show the best catalytic performance in the combustion of non-halogenated VOCs. The noble catalysts which have been investigated include platinum, palladium, ruthenium, iridium, gold, and silver (Table 5) [2,41-44]. Due to their size-dependence catalytic properties and high price, noble metal catalysts are often supported on porous supports, such as $\gamma-\mathrm{Al}_{2} \mathrm{O}_{3}, \mathrm{SiO}_{2}$, zeolite, and other non-metal oxides to increase the dispersion of noble metal nanoparticles and surface area, which can improve the catalytic efficiency of noble metal catalysts [106-111]. Catalysts are often supported on the substrate, which is in the form of a monolith or honeycomb material, such as cordierite, aluminum, and stainless steel [112]. Noble catalysts display high efficiencies for VOC removal at a lower temperature than other kinds of catalysts. Pt shows the best catalytic combustion of VOCs, exhibiting equal removal efficiencies at operating temperatures of up to $100 \mathrm{~K}$ lower than those used for other noble metals [42,44]. Two key factors were found to influence the catalytic performance of alumina supported $\mathrm{Pt}$, dispersion and loading amount, with an increase in either of these properties associated with an obvious improvement of catalytic activity $[106,113,114]$. The metal particle size is also an important factor that influences the catalytic activity. Changes to the active metal particle size can also enhance catalytic performance. For instance, when the crystalline size of alumina supported Pt increased from 1.0 to $15.5 \mathrm{~nm}$, the oxidation rate was found to increase by a factor of 10 [115]. The same phenomenon was also found in the oxidation of propylene over alumina supported $\mathrm{Pt}$ and Pd catalysts.

An important factor in the performance of noble metal catalysts for VOC combustion is specificity; in other words, the target VOCs molecules. For instance, P. Papaefthimiou et al. found that Pt and Pd supported on alumina showed good performance for the oxidation of benzene and butanol, but not ethyl acetate, with Pd generally outperforming Pt [57]. M. J. Patterson et al. discovered that alumina supported rhodium is the most active noble metal for 1-hexene, but not for aromatics, while benzene can be decomposed the most easily on platinum, and palladium showed the best catalytic performance in abatement of toluene [116]. The impact of certain non-VOC species in the waste stream can also impact performance, where these species can potentially 'poison' the catalyst or cause side reactions to occur. One example is the presence of $\mathrm{CO}$, which was found to have little effect on the performance of Pd catalysts, but significantly inhibit the activity over Pt [117]. 
Table 5. Noble metal catalysts for catalytic combustion of VOCs.

\begin{tabular}{|c|c|c|c|c|}
\hline Catalyst & VOCs & Catalytic Performance & Remarks & Catalytic Mechanism \\
\hline $0.3 \% \mathrm{Pt} / \gamma-\mathrm{Al}_{2} \mathrm{O}_{3}$ & $\begin{array}{l}\text { Butanol } \\
\text { Benzene }\end{array}$ & $\begin{array}{c}\mathrm{T}_{95}: 200^{\circ} \mathrm{C} \\
220^{\circ} \mathrm{C}\end{array}$ & - & - \\
\hline Pd-AuFeCeIM & Benzene & $\mathrm{T}_{100}: 200^{\circ} \mathrm{C}$ & - & - \\
\hline $\mathrm{Pd}$ (shell)-Au(core)/ $/ \mathrm{TiO}_{2}$ & Toluene & $\begin{array}{l}\mathrm{T}_{50}: 220^{\circ} \mathrm{C} \\
\mathrm{T}_{90}: 230^{\circ} \mathrm{C}\end{array}$ & - & Langmuir-Hinshelwood mechanism. \\
\hline $\mathrm{Au}-\mathrm{Pd} / \mathrm{CeO}_{2}$ & Toluene & $\begin{array}{l}\mathrm{T}_{50}: 120{ }^{\circ} \mathrm{C} \\
\mathrm{T}_{90}: 150{ }^{\circ} \mathrm{C}\end{array}$ & - & $\begin{array}{l}\text { Due to the synergistic effect between } \mathrm{Au} \text { and } \mathrm{Pd} \\
\text { nanoclusters, } \mathrm{Au}-\mathrm{Pd} / \mathrm{CeO}_{2} \text { bimetallic catalysts are } \\
\text { much better than } \mathrm{Au} \text { and } \mathrm{Pd} \text { single metal catalysts. }\end{array}$ \\
\hline $\mathrm{Au}-\mathrm{Ag} / \mathrm{CeO}_{2}$ & Isopropanol & $\begin{array}{l}\mathrm{T}_{50}: 105^{\circ} \mathrm{C} \\
\mathrm{T}_{90}: 158^{\circ} \mathrm{C}\end{array}$ & - & MVK redox mechanism. \\
\hline $\mathrm{Pt}-\mathrm{Au} / \mathrm{Ce}-\mathrm{Al}$ & $\mathrm{CH}_{3} \mathrm{SSCH}_{3}$ & $\begin{array}{l}\mathrm{T}_{50}: 425^{\circ} \mathrm{C} \\
\mathrm{T}_{90}: 480^{\circ} \mathrm{C}\end{array}$ & - & $\begin{array}{l}\text { The addition of Au improved the selectivity of } \\
\text { ceria-containing catalysts by decreasing the formation } \\
\text { of byproducts due to lower amount of reactive oxygen } \\
\text { after the Au addition. }\end{array}$ \\
\hline $\mathrm{Cu}-\mathrm{Au} / \mathrm{Ce}-\mathrm{Al}$ & $\mathrm{CH}_{3} \mathrm{SSCH}_{3}$ & $\begin{array}{l}\mathrm{T}_{50}: 275^{\circ} \mathrm{C} \\
\mathrm{T}_{90}: 370^{\circ} \mathrm{C}\end{array}$ & - & - \\
\hline $\mathrm{Ag} / \mathrm{NiOx}-\mathrm{MnO}_{2}$ & O-xylene & $\begin{array}{l}\mathrm{T}_{50}: 145^{\circ} \mathrm{C} \\
\mathrm{T}_{90}: 190^{\circ} \mathrm{C}\end{array}$ & $\begin{array}{l}\text { The catalytic activity decreased } \\
\text { with the addition of water } \\
\text { vapor, but recovered after the } \\
\text { removal of water vapor. }\end{array}$ & $\begin{array}{c}\text { Mars-Van Krevelen mechanism. (i) Electrophilic } \mathrm{O}_{1} \\
\text { +aromatic ring } \rightarrow \text { maleate; (ii) } \mathrm{O}_{2}+\text { Cat. } \rightarrow \text { electrophilic } \\
\text { oxygen species }\left(\mathrm{O}_{2}{ }^{2-}, \mathrm{O}^{2-}, \mathrm{O}^{-}\right) \text {, electrophilic oxygen } \\
\text { species }+ \text { aromatic ring } \rightarrow \text { maleate, carbonate }+\left(\mathrm{O}_{2},\right. \\
\left.\mathrm{O}_{2}{ }^{2-}, \mathrm{O}^{2-}, \mathrm{O}^{-} \text {or } \mathrm{O}_{2}\right)+\mathrm{Ag} \rightarrow \text { nucleophilic oxygen, } \\
\text { nucleophilic oxygen }+ \text { maleate and carboxylates } \rightarrow \mathrm{CO}_{2} \\
\text { and } \mathrm{H}_{2} \mathrm{O} \text {. }\end{array}$ \\
\hline $2.3 \mathrm{wt} \% \mathrm{Pt} / 3 \mathrm{DOM}-\mathrm{Mn}_{2} \mathrm{O}_{3}$ & Toluene & $\begin{array}{l}\mathrm{T}_{50}: 165^{\circ} \mathrm{C} \\
\mathrm{T}_{90}: 194^{\circ} \mathrm{C}\end{array}$ & - & $\begin{array}{c}\text { A first-order reaction mechanism. Toluene } \rightarrow \text { cat. } \\
\text { surface } \rightarrow \text { benzyl alcohol } \rightarrow \text { benzoic acid and } \\
\text { benzaldehyde } \rightarrow \text { (rise temperature }) \text { maleic } \\
\text { anhydride } \rightarrow \mathrm{CO}_{2} \text { and } \mathrm{H}_{2} \mathrm{O} \text {. }\end{array}$ \\
\hline
\end{tabular}


Transition metal oxides can be utilized as both supports and promoters for noble metal catalysts. $\mathrm{Pt}, \mathrm{Pd}, \mathrm{Ru}$, Au supported on $\mathrm{MgO}, \mathrm{SnO}_{2}, \mathrm{Co}_{3} \mathrm{O}_{4}, \mathrm{NiO}, \mathrm{TiO}_{2}, \mathrm{CeO}_{2}, \mathrm{La}_{2} \mathrm{O}_{3}, \mathrm{ZrO}_{2}$ or $\mathrm{PrO}_{2}$ have been explored for the oxidation of toluene, benzene, xylene, propene, light alkane, ethanol, propanol, butanol, formaldehyde, acetone, and acetic acid, respectively [42]. The role of the transition metal oxides is not only to supply a large surface area to disperse the noble metal particles, but in some cases, it can also improve the catalytic performance of noble metal particles by enhancing the mobility of lattice oxygen species. Previous studies showed that active oxygen species formed on cobalt oxide spinel-type crystallites can enhance the catalytic oxidation over PdO supported on alumina $[118,119]$.

The reduction properties also influence the oxidation ability of supported noble metal catalysts. T. Mitsui et al. prepared $\mathrm{SnO}_{2}, \mathrm{CeO}_{2}$, and $\mathrm{ZrO}_{2}$ supported $\mathrm{Pt}$ and Pd catalysts for the abatement of acetaldehyde [120]. These prepared catalysts were treated in an $\mathrm{H}_{2} / \mathrm{N}_{2}$ flow and calcined in the atmosphere. The results showed that the $\mathrm{SnO}_{2}$ supported $\mathrm{Pt}$ and $\mathrm{Pd}$ showed the best catalytic performance among the calcined catalysts in the atmosphere, while after treatment in an $\mathrm{H}_{2} / \mathrm{N}_{2}$ flow, the catalytic activity of $\mathrm{SnO}_{2}$ supported $\mathrm{Pt}$ and $\mathrm{Pd}$ decreased due to the formation of inactive inter-metallic phases ( $\mathrm{PtSn}$ and $\mathrm{Pd}_{3} \mathrm{Sn}_{2}$ ). In contrast, $\mathrm{CeO}_{2}$ and $\mathrm{ZrO}_{2}$ supported catalysts showed the improved catalytic activity after reduction. In the elimination of formaldehyde over a $\mathrm{TiO}_{2}$-supported catalyst, $\mathrm{Pt} / \mathrm{TiO}_{2}$ showed a superior catalytic performance to $\mathrm{Rh} / \mathrm{TiO}_{2}, \mathrm{Pd} / \mathrm{TiO} \mathrm{O}_{2}, \mathrm{Au} / \mathrm{TiO}_{2}$, and neat $\mathrm{TiO}_{2}$ [121]. Other research showed that a series of supported $\mathrm{Pt}, \mathrm{Pd}$, and $\mathrm{Au}$ catalysts can even partially eliminate formaldehyde at room temperature [122].

Recently, the use of $\mathrm{MnO}_{\mathrm{x}}$ based materials as catalyst supports has gained attention. In one instance, supporting $\mathrm{Ag}$ on $\mathrm{NiO}-$ doped $\mathrm{MnO}_{2}$ showed a high activity towards the combustion of o-xylene [89]. The improvement in xylene oxidation was attributed to the enhanced oxygen activation and mobility afforded to the catalyst support via the addition of $\mathrm{NiO}$ and $\mathrm{Ag}$. Wenbo Pei et al. explored the use of ordered, mesoporous $\mathrm{Mn}_{2} \mathrm{O}_{3}$ supports with embedded Pt particles for the catalytic combustion of toluene [66]. They found that the strong interaction between $\mathrm{Pt}$ and $\mathrm{Mn}_{2} \mathrm{O}_{3}$ in the ordered structure improves the activity and stability of the catalyst.

The single noble metal catalysts cannot satisfy the requirements of VOCs combustion. Therefore, some mixed noble metal catalysts have been developed to combine the advantages of different noble metal catalysts, such as Pt-Au, Cu-Au, and Pd-Au. T. Tabakova et al. found that the Pd deposition on the deposited gold showed the best catalytic performance for benzene combustion, which was totally eliminated at $200{ }^{\circ} \mathrm{C}$. It also showed good stability [57]. M. Hosseini et al. showed that the deposition of palladium on aurum supported on $\mathrm{TiO}_{2}\left(\mathrm{Pd}(\right.$ shell $\left.)-\mathrm{Au}(\mathrm{core}) / \mathrm{TiO}_{2}\right)$ can significantly improve the catalytic activity for oxidation of toluene and propylene [67]. Der Shing Lee et al. deposited Au-Pd bimetallic nanoparticles on $\mathrm{CeO}_{2}$ for toluene degradation, which showed a much better catalytic performance than $\mathrm{Au} / \mathrm{CeO}_{2}$ and $\mathrm{Pd} / \mathrm{CeO}_{2}$ catalysts due to the synergistic effect of gold and palladium [68]. The addition of non-noble metals also can improve the catalytic activity of noble metal catalysts. Roberto Fiorenza et al. prepared $\mathrm{Au}-\mathrm{Ag} / \mathrm{CeO}_{2}$ and $\mathrm{Au}-\mathrm{Cu} / \mathrm{CeO}$ bimetallic catalysts for alcohol oxidation and $\mathrm{CO}$ oxidation. These two catalysts showed higher selectivity for intermediate products higher $\mathrm{CO}$ conversion at a low temperature $\left(100^{\circ} \mathrm{C}\right)$ than $\mathrm{Au} / \mathrm{CeO}_{2}$ [93]. The addition of $\mathrm{Au}$ also can improve the performance of $\mathrm{Al}_{2} \mathrm{O}_{3}$ supported $\mathrm{Cu}$-Pt catalysts in DMDS oxidation [81].

Noble metal catalysts showed a high catalytic activity and remarkable thermal stability in catalytic elimination of VOCs. However, the use of noble metal catalysts is also associated with distinct disadvantages. Firstly, the high cost of noble metal limits their application in the industrial abatement of VOCs. Secondly, the presence of chlorine, sulfur, $\mathrm{CO}$, and water can suppress the catalytic performance significantly [42,123-125]. The regeneration and recycling of noble metal catalysts poisoned by $\mathrm{Cl}$ and $\mathrm{S}$ is difficult, so they are not suited to the treatment chlorine and sulfur-containing VOCs. In fact, the release of chlorine and sulfur containing VOCs is particularly common in pharmaceutical production processes, so chlorine- and sulfur-containing VOCs need to be removed prior to the treatment by noble metal catalysts, which will further increase the cost of waste gas purification. 


\subsubsection{Non-Noble Metal Catalysts}

To address the cost of noble metals, non-noble metal oxides catalysts were developed for the abatement of VOCs. The materials which have been studied as non-noble metals include the derivatives of transition metals and rare earth elements, such as $\mathrm{Ti}, \mathrm{Cu}, \mathrm{Mn}, \mathrm{Al}, \mathrm{Ce}, \mathrm{Co}, \mathrm{Fe}, \mathrm{Cr}$, and V (Table 6) [1,126-129]. Although transition metal oxides catalysts generally showed a lower catalytic activity than noble metal catalysts for the oxidation of VOCs, they have many advantages, such as a resistance to chlorine and sulfur poisoning, tunable material properties, low cost, long on-stream lifetime, easy regeneration, and low environmental impact. The non-noble metal oxides catalysts applied and studied in VOCs abatement include $\mathrm{CuO}_{\mathrm{x}}, \mathrm{MnO}_{2}, \mathrm{FeO}_{\mathrm{x}}, \mathrm{NiO}_{\mathrm{x}}, \mathrm{CrO}_{\mathrm{x}}$, and $\mathrm{CoO}_{\mathrm{x}}$.

The non-noble metal oxides catalyst systems which we will discuss include ones in which the metal oxide is both supported or unsupported. Due to the presence of mobile oxide species in lattice, $\mathrm{Co}_{2} \mathrm{O}_{3}$ displays excellent reduction and oxidation abilities. As such, studies have shown $\mathrm{Co}_{2} \mathrm{O}_{3}$ to be one of the best catalysts used for the combustion of benzene, toluene, propane, 1,2-dichloroethane, and 1,2-dichlorobenzene [130-133]. The catalytic activity of $\mathrm{Co}_{2} \mathrm{O}_{3}$ is determined by the method of preparation, treatment conditions, and surface area.

$\mathrm{MnO}_{2}$ is another commonly used metal oxide catalyst, which has been applied and studied in the abatement of n-hexane, acetone, benzene, ethanol, toluene, propane, trichloroethene, ethyl acetate, and $\mathrm{NO}_{x}[133-139]$. The catalytic activity can be tuned by the preparation method and depends on the structure, surface area, support materials, and oxidation states of catalysts. In the catalytic combustion of ethyl acetate and hexane, $\mathrm{MnO}_{2}$ even achieved a better activity than $\mathrm{Pt} / \mathrm{TiO}_{2}$ [140]. Yonghui Wei et al. removed the La atoms from the $\mathrm{LaMnO}_{3}$ perovskite to prepare $\mathrm{MnO}_{2}$ with a high surface area $\left(>150 \mathrm{~m}^{2} / \mathrm{g}\right.$ ), upon which it showed excellent catalytic activity in the oxidation of toluene [96]. Zhang Kai et al. synthesized the nano-cubic $\mathrm{MnO}_{\mathrm{x}}$ which has a large specific surface area, many oxygen vacancies, and good low temperature reducibility. The conversion of toluene via combustion was more than $90 \%$ at $350{ }^{\circ} \mathrm{C}$ [95]. Xueqin Yang et al. found that the acid treatment did not change the morphology of the catalyst, but could improve the oxidation ability of the catalyst by increasing the number of $\mathrm{Mn}^{4+}$ species and structural defects on the surface of the catalyst [69]. A common theme throughout the implementation of Mn-based metal oxide catalysts for VOC oxidation is the availability and mobility of oxygen within the $\mathrm{MnO}_{\mathrm{x}}$, which is attributed to the oxidation and reduction ability of the Mn afforded by the multiple oxidation states in which it can exist.

Copper oxides are another kind of efficient catalysts used in total oxidation of methane, methanol, ethanol, and acetaldehyde [141,142]. The main factors which influence the catalytic activity are the $\mathrm{Cu}$ oxidation state and the availability of lattice oxygen. The addition of other metal oxides, such as $\mathrm{CeO}_{2}$, can enhance the catalytic ability noticeably [133].

Chromium oxides are also promising oxidation catalysts, especially for the combustion of halogenated VOCs $[124,133,143]$. For chromium oxides, highly crystalline samples showed a better catalytic activity than amorphous ones [144]. Rotter et al.'s research showed that, when using $\mathrm{TiO}_{2}$ as a support material, chromium oxides achieved higher catalytic oxidation of trichloroethylene than manganese oxide, cobalt oxide, and iron oxide [82]. Chromium has also been successfully supported on silica, alumina, porous carbon, and clay to eliminate pollutants such as carbon tetrachloride, chloromethane, trichloroethylene, ethyl chloride, chlorobenzene, and perchloroethylene [142]. However, chromium oxides also suffer deactivation due to the reaction between chromium and chlorine to form $\mathrm{Cr}_{2} \mathrm{Cl}_{2}[145,146]$.

$\mathrm{CeO}_{2}$ is a widely used catalyst in oxidation reactions due to its strong interactions with other metals, high oxygen storage capacity, and ready shuttling between the $\mathrm{Ce}^{3+}$ and $\mathrm{Ce}^{4+}$ states [147-149]. Dai et al. compared the removal of chlorinated alkanes and alkylenes over $\mathrm{CeO}_{2}$ [83]. The results showed that $\mathrm{CeO}_{2}$ is more efficient when it comes to oxidizing chlorinated alkanes than chlorinated alkylenes. $\mathrm{CeO}_{2}$ also faced the deactivation problem due to the absorption of $\mathrm{Cl}_{2}$ and $\mathrm{HCl}$ on the surface [150], so the design of chlorine resistance metal oxide catalysts is still a challenge which must be overcome. 
Vanadium oxides were also developed to decompose chlorinated VOCs, such as polychlorinated pollutants and dichlorobenzene due to its tolerance of chlorine and sulfur compounds [151]. The presence of water can enhance and suppress the catalytic activity of $\mathrm{V}_{2} \mathrm{O}_{5}$ via the removal of surface absorbed chlorine and reduction of active sites, respectively [152]. Other non-noble metal oxides were also investigated for abatement of $\mathrm{VOCs}$, such as $\mathrm{NiO}$ and $\mathrm{FeO}_{\mathrm{x}}$, which require further improvement of catalytic efficiency [153].

The above discussion suggests that the use of a single metal oxide as VOC oxidation catalysts is too often plagued by either a low catalytic activity or catalyst poisoning. Thus, focus has shifted to the development of mixed metal oxide catalysts such as $\mathrm{Mn}-\mathrm{Ce}, \mathrm{Mn}-\mathrm{Cu}, \mathrm{Co}-\mathrm{Ce}, \mathrm{Sn}-\mathrm{Ce}, \mathrm{Mn}-\mathrm{Co}$, and $\mathrm{Ce}-\mathrm{Cu}$ oxides $[119,154-157]$. The logic here is that combining two metal oxides with different materials and catalytic properties allows for a synergistic enhancement in performance. The previous studies showed that the rate determining step of VOC catalytic combustion was the oxygen removal from the catalysts lattice [119], so the goal of mix-metal oxides catalysts design was the enhancement of the lattice oxygen species availability.

The addition of copper into $\mathrm{CeO}_{2}$ can promote the catalytic efficiency due to a synergistic effect, leading to a highly efficient decomposition of ethyl acetate, ethanol, propane, benzene, and toluene [158-160]. $\mathrm{MnO}_{\mathrm{x}}-\mathrm{CeO}_{2}$ has been applied for the destruction of ethanol, formaldehyde, hexane, phenol, ethyl acetate, and toluene [155,161-164]. Mn-Co oxides catalysts also showed improved catalytic activity relative to either $\mathrm{MnO}_{x}$ or $\mathrm{Co}_{2} \mathrm{O}_{3}$ in the combustion of ethyl acetate and n-hexane [100]. $\mathrm{CeO}_{2}-\mathrm{CrO}_{x}$ showed excellent catalytic activity for the decomposition of chlorinated VOCs [165], while the removal of chlorobenzene over $\mathrm{MnO}_{\mathbf{x}}-\mathrm{TiO}_{2}$ and $\mathrm{MnO}_{\mathrm{x}}-\mathrm{TiO}_{2}-\mathrm{SnO}_{\mathrm{x}}$ showed much better catalytic performance than not only the individual oxides, but also achieved removal efficiencies on par with noble metal catalysts [166]. A three-dimensional ordered mesoporous material of mixed cerium-manganese oxide was prepared for the efficient catalytic combustion of chlorine-containing VOCs due to its large specific surface, enriched $\mathrm{Ce}^{3+}$ content, oxygen vacancies, active oxygen species, and acidic sites. It showed good water resistance and high airspeed applicability. However, catalyst deactivation caused by inorganic chlorine adsorption still occurred [90]. Layered copper manganese oxide has been prepared for the catalytic combustion of $\mathrm{CO}$ and VOCs, which showed efficient activity due to the interfacial structure of mixed phases and the formation of the $\mathrm{Cu}^{2+}-\mathrm{O}^{2-}-\mathrm{Mn}^{4+}$ entity [58]. Acidic sites can be provided by support to prevent the decrease of catalytic activity. $\mathrm{CeO}_{2} @ \mathrm{SiO}_{2}$ was prepared to catalyze the combustion of 1,2-dichloroethane. $\mathrm{SiO}_{2}$ can provide weak acid sites, as well as promote the adsorption and activation of 1,2-dichloroethane and the desorption of generated $\mathrm{HCl}$ [85].

A vast number of preparation methods exist for the synthesis of mixed metal oxide catalysts, including thermal decomposition, impregnation, co-precipitation, and the sol-gel method [167-169]. The selection of preparation methods depends on the properties of catalysts and the application situation. Furthermore, as discussed above, complex ordered microporous, multilayer or core-shell structures have recently been applied to catalytic oxidation processes to access properties which come from having a highly controlled particle composition and morphology. The chance to alter not only the metal centers present within mixed metal oxide systems, but also the relative metal ratios. 
Table 6. Non-noble metal catalysts for catalytic combustion of VOCs.

\begin{tabular}{|c|c|c|c|c|}
\hline Refer & Catalyst & VOCs & Catalytic Performance & Remarks \\
\hline [69] & $\mathrm{Mn}_{2} \mathrm{O}_{3}$ & Toluene & \multirow{4}{*}{$\begin{array}{c}\mathrm{T}_{50}: 231^{\circ} \mathrm{C} \\
\mathrm{T}_{90}: 239^{\circ} \mathrm{C} \\
\mathrm{T}_{50}: 283^{\circ} \mathrm{C} \\
\mathrm{T}_{90}: 317^{\circ} \mathrm{C} \\
\mathrm{T}_{50}: 340^{\circ} \mathrm{C} \\
\mathrm{T}_{90}: 378^{\circ} \mathrm{C} \\
\mathrm{T}_{90}: 260^{\circ} \mathrm{C} \\
\mathrm{T}_{90}: 160^{\circ} \mathrm{C} \\
\text { GHSV }=15,000 \mathrm{~mL} /(\mathrm{g} \cdot \mathrm{h}) \\
\mathrm{T}_{50}: 291^{\circ} \mathrm{C} \\
\mathrm{T}_{90}: 347^{\circ} \mathrm{C} \\
\text { GHSV }=7500 \mathrm{~mL} /(\mathrm{g} \cdot \mathrm{h}) \\
\mathrm{T}_{90}: 224^{\circ} \mathrm{C} \\
\text { GHSV }=30,000 \mathrm{~mL} /(\mathrm{g} \cdot \mathrm{h}) \\
\mathrm{T}_{90}: 360^{\circ} \mathrm{C}\end{array}$} & - \\
\hline [82] & $\mathrm{Pt} / \mathrm{CrOOH}$ & $\begin{array}{l}\text { Dichloroethane } \\
\text { Chlorobenzene }\end{array}$ & & - \\
\hline [83] & $\mathrm{CeO}_{2}$ & $\begin{array}{l}\text { Tetrachloroethylene } \\
\text { dichloromethane }\end{array}$ & & $\begin{array}{l}\text { The addition of } 3 \%(\mathrm{v} / \mathrm{v}) \text { water can obviously inhibit the } \\
\text { catalytic decomposition of } \mathrm{VOCs} \text { on } \mathrm{CeO}_{2} \text {. }\end{array}$ \\
\hline$[90]$ & CeMn30 & O-dichlorobenzene & & $\begin{array}{l}\text { The addition of low concentration of water vapor is } \\
\text { beneficial to the early reaction, but will weaken the catalytic } \\
\text { activity of the catalyst. }\end{array}$ \\
\hline [96] & $\mathrm{MnO}_{2}$ & VOCs & $\begin{array}{l}\mathrm{T}_{50}: 233^{\circ} \mathrm{C} \\
\mathrm{T}_{90}: 256^{\circ} \mathrm{C}\end{array}$ & - \\
\hline [95] & $\begin{array}{l}\text { Nanocubic } \\
\mathrm{MnO}_{2}\end{array}$ & Methane & $\begin{array}{l}\mathrm{T}_{50}: 293^{\circ} \mathrm{C} \\
\mathrm{T}_{90}: 350^{\circ} \mathrm{C}\end{array}$ & - \\
\hline
\end{tabular}




\subsubsection{Perovskite Catalysts}

Perovskite-type oxides are a kind of composite oxides which have a similar structure with $\mathrm{CaTiO}_{3}$, and can be expressed by $\mathrm{ABO}_{3}$. The common way to modify the perovskite catalysts is replacement of the cation $\mathrm{B}$ by $\mathrm{B}^{\prime}$ to tune the redox ability or enhance the stability [1]. With the replacement of the cation $B$, the crystal lattice would be distortion which leads to the enhancement of redox ability and improvement of stability. The most commonly used perovskite for catalytic combustion of VOCs is $\mathrm{LaBO}_{3}$, in which $\mathrm{B}$ can be $\mathrm{Co}, \mathrm{Fe}, \mathrm{Ni}, \mathrm{Mn}$, and $\mathrm{Sr}$ (Table 7) $[60,97,170,171]$. Huang et al. used Sr partially replaced $\mathrm{La}$ in $\mathrm{LaCoO}_{3}$ for catalytic combustion of propyl alcohol, toluene, and cyclohexane [70]. The results showed that the doped $\mathrm{LaCoO}_{3}$ showed a better catalytic performance than the undoped one, and the modified catalysts were stable in the reaction. R. Spinicci et al. compared the catalytic activity of $\mathrm{LaMnO}_{3}$ and $\mathrm{LaCoO}_{3}$ for catalytic combustion of acetone, isopropanol, and benzene [60]. They suggested that $\mathrm{LaMnO}_{3}$ showed a better performance than $\mathrm{LaCoO}_{3}$. In oxidation of isopropanol, acetone was the intermediate product. The surface oxygen species played a key role in this process. The increase of oxygen pressure is positive for the catalytic combustion of VOCs over these perovskite catalysts. G. Sinquin et al. applied $\mathrm{LaMnO}_{3}$ and $\mathrm{LaCoO}_{3}$ for the catalytic combustion of chlorinated VOCs, such as $\mathrm{CH}_{2} \mathrm{Cl}_{2}$ and $\mathrm{CCl}_{4}$. $\mathrm{LaMnO}_{3}$ showed a better chlorine resistance than $\mathrm{LaCoO}_{3}$ [97]. Mihai Alifanti et al. supported $\mathrm{LaCoO}_{3}$ on cerium-zirconium oxides $\left(\mathrm{Ce}_{1-\mathrm{x}} \mathrm{Zr}_{\mathrm{x}} \mathrm{O}_{2}, \mathrm{x}=0-0.3\right)$ for the catalytic combustion of benzene and toluene [172]. The results showed that all the supported catalysts showed a better performance than $\mathrm{Ce}_{1-\mathrm{x}} \mathrm{Zr}_{\mathrm{x}} \mathrm{O}_{2}$ and $20 \%$ loaded $\mathrm{LaCoO}_{3}$ showed about 10 times higher catalytic activity than $\mathrm{LaCoO}_{3}$ for toluene oxidation due to its large surface area and good oxygen mobility. S. I. Suárez-Vázquez et al. synthesized $\operatorname{SrTi}_{1-\mathrm{x}} \mathrm{B}_{\mathrm{x}} \mathrm{O}_{3}(\mathrm{~B}=\mathrm{Mn}, \mathrm{Cu})$ for toluene destruction [71]. Mn could replace $\mathrm{Ti}$ and enter the perovskite structure, while $\mathrm{Cu}$ could not. The $\mathrm{Mn}$ doped catalysts showed the highest catalytic activity and can completely decompose toluene to $\mathrm{CO}_{2}$ at a temperature lower than $350^{\circ} \mathrm{C}$. Perovskite also can be prepared from solid waste such as the obsoleting lithium battery. Mingming Guo et al. prepared manganese-based perovskite catalyst from the waste lithium battery for catalytic combustion of toluene, which showed a better catalytic activity than pure manganese perovskite catalyst due to more $\mathrm{Mn}^{4+}$ ions and lattice oxygen species, as well as high specific surface area [72]. In order to increase the amounts of active sites, Junxuan Yao et al. removed the La ions from $\mathrm{LaCoO}_{3}$ to obtain the disordered $\mathrm{Co}_{3} \mathrm{O}_{4}$. It showed a better catalytic activity for propane combustion than the one prepared by other methods [94]. To further improve the catalytic activity, $\gamma-\mathrm{MnO}_{2}$ was calcined on the surface of $\mathrm{SmMnO}_{3}$ which had a large specific surface area, high $\mathrm{Mn}^{4+} / \mathrm{Mn}^{3+}$ and $\mathrm{O}_{\text {latt }} / \mathrm{O}_{\text {ads }}$. Compared with $\mathrm{SmMnO}_{3}$, it showed a better catalytic activity and stability (10 vol\% water) in the process of catalytic reaction [61]. Jingsi Yang et al. assembled the $\mathrm{LaMnO}_{3}$ perovskite in $\mathrm{MnO}_{2}$ and adjusted $\mathrm{La} / \mathrm{Mn}$ to 15 . The redox ability of the catalyst was improved by enhancing the interaction between the active phase and the support [73]. The ratio of citric acid and metal ion $\left(\mathrm{La}^{3+} \mathrm{Mn}^{2+}\right)$ was also tested to find out the best composite of perovskite catalysts. Zakaria Sihaib et al. prepared $\mathrm{LaMnO}_{3}$ with different ratios. The results show that the catalyst with the ratio of 0.5 to 1.5 has the best catalytic performance and the amount of citric acid affects the specific surface area of perovskite catalyst [74]. Li Wang et al. added $\mathrm{Sr}$ into the $\mathrm{LaMnO}_{3}$ to prepare $\mathrm{La}_{0.5} \mathrm{Sr}_{0.5} \mathrm{MnO}_{3}$. The amount of $\mathrm{Mn}^{4+}$ and the oxidation ability of vinyl chloride has been improved after $\mathrm{HCl}$ modification [104]. The perovskite catalysts showed a good catalytic activity for combustion of VOCs at low temperature due to their tunable redox property by replacing the B atom. However, they also have some disadvantages, such as low thermal stability. The catalytic activity and stability of perovskite catalysts need to be further improved. 
Table 7. Perovskite catalysts for catalytic combustion of VOCs.

\begin{tabular}{|c|c|c|c|c|c|}
\hline Refer & Catalyst & VOCs & Catalytic Performance & Remarks & Catalytic Mechanism \\
\hline$[58]$ & $\begin{array}{l}\text { Layered copper } \\
\text { manganese oxide } \\
(\mathrm{LCMO})\end{array}$ & $\begin{array}{c}\text { CO } \\
\text { Benzene } \\
\text { Toluene } \\
\text { O-xylene }\end{array}$ & $\begin{array}{c}\mathrm{T}_{50}: 62{ }^{\circ} \mathrm{C} \\
\mathrm{T}_{90}: 76^{\circ} \mathrm{C} \\
\mathrm{T}_{50}: 218^{\circ} \mathrm{C} \\
\mathrm{T}_{90}: 240^{\circ} \mathrm{C} \\
\mathrm{T}_{50}: 187^{\circ} \mathrm{C} \\
\mathrm{T}_{90}: 207^{\circ} \mathrm{C} \\
\mathrm{T}_{50}: 213^{\circ} \mathrm{C} \\
\mathrm{T}_{90}: 227^{\circ} \mathrm{C}\end{array}$ & $\begin{array}{l}\text { The introduction of water vapor had } \\
\text { no significant effect on the catalyst. } \\
\text { The catalyst activity decreased about } \\
30 \% \text { after } \mathrm{SO}_{2} \text { was added. } \\
\text { The addition of } \mathrm{CO}_{2} \text { has no effect on } \\
\text { the catalytic activity of the catalyst. }\end{array}$ & $\begin{array}{l}\text { The larger surface area and the } \\
\text { formation of } \mathrm{Cu}^{2+}-\mathrm{O}^{2-}-\mathrm{Mn}^{4+} \text { entities } \\
\text { at the interface between } \mathrm{CuO} \text { and } \\
\text { layered } \mathrm{MnO}_{2} \text { promoted the } \mathrm{CO} \text { and } \\
\text { VOCs oxidation. }\end{array}$ \\
\hline [59] & $\mathrm{LaCoO}_{3}$ & $\begin{array}{l}\text { Benzene } \\
\text { Toluene }\end{array}$ & $\begin{array}{l}\mathrm{T}_{50}: 330^{\circ} \mathrm{C} \\
\mathrm{T}_{50}: 244^{\circ} \mathrm{C}\end{array}$ & $\begin{array}{l}\text { Water vapor has no significant effect } \\
\text { on the catalytic performance of } \\
\text { catalyst. }\end{array}$ & $\begin{array}{c}\text { Factors improve activity: (i) The larger } \\
\text { exposed surface, (ii) the composition } \\
\text { of the support provides increased } \\
\text { oxygen mobility. }\end{array}$ \\
\hline$[60]$ & $\begin{array}{l}\mathrm{LaMnO}_{3} \\
\mathrm{LaCoO}_{3}\end{array}$ & $\begin{array}{l}\text { Isopropanol } \\
\text { Benzene } \\
\text { Propyl alcohol } \\
\text { Isopropanol } \\
\text { Benzene } \\
\text { Propyl alcohol }\end{array}$ & $\begin{array}{c}\mathrm{T}_{50}: \\
216^{\circ} \mathrm{C} \\
301^{\circ} \mathrm{C} \\
203^{\circ} \mathrm{C} \\
\mathrm{T}_{90}: \\
237^{\circ} \mathrm{C} \\
323^{\circ} \mathrm{C} \\
222^{\circ} \mathrm{C}\end{array}$ & - & - \\
\hline$[61]$ & $\gamma-\mathrm{MnO} 2 / \mathrm{SmMnO}_{3}$ & $\begin{array}{c}\text { Toluene } \\
\text { Benzene } \\
\text { O-xylene } \\
\text { Ethylbenzene }\end{array}$ & $\begin{array}{l}\mathrm{T}_{50}: 187^{\circ} \mathrm{C} \\
\mathrm{T}_{90}: 208^{\circ} \mathrm{C} \\
\mathrm{T}_{50}: 213^{\circ} \mathrm{C} \\
\mathrm{T}_{90}: 226^{\circ} \mathrm{C} \\
\mathrm{T}_{50}: 232^{\circ} \mathrm{C} \\
\mathrm{T}_{90}: 250^{\circ} \mathrm{C} \\
\mathrm{T}_{50}: 201^{\circ} \mathrm{C} \\
\mathrm{T}_{90}: 217^{\circ} \mathrm{C}\end{array}$ & - & $\begin{array}{l}\text { Toluene-Mn ions }+ \text { surface reactive } \\
\text { oxygen } \rightarrow \text { benzaldehyde } \rightarrow \text { benzoic } \\
\text { acid } \rightarrow \text { chain carboxylic acids } \rightarrow \mathrm{CO}_{2} \text {. }\end{array}$ \\
\hline
\end{tabular}


Table 7. Cont.

\begin{tabular}{|c|c|c|c|c|c|}
\hline Refer & Catalyst & VOCs & Catalytic Performance & Remarks & Catalytic Mechanism \\
\hline [70] & $\mathrm{La}_{0.8} \mathrm{Sr}_{0.2} \mathrm{CoO}_{3}$ & $\begin{array}{l}\text { toluene } \\
\text { Cyclohexane } \\
\text { Propyl alcohol }\end{array}$ & $\begin{array}{l}\mathrm{T}_{50}:<160{ }^{\circ} \mathrm{C} \\
\mathrm{T}_{99}: 300{ }^{\circ} \mathrm{C} \\
\mathrm{T}_{50}: 180{ }^{\circ} \mathrm{C} \\
\mathrm{T}_{99}: 260{ }^{\circ} \mathrm{C} \\
\mathrm{T}_{90}: 160^{\circ} \mathrm{C}\end{array}$ & - & $\begin{array}{l}\text { Partial substitution of strontium }(\mathrm{Sr}) \\
\text { for lanthanum (La) greatly increased } \\
\text { the oxygen vacancies in the surface } \\
\text { regions to enhance the catalytic } \\
\text { activity. }\end{array}$ \\
\hline$[71]$ & $\begin{array}{l}\mathrm{SrTi}_{1-\chi} \mathrm{Cu}_{X} \mathrm{O}_{3} \\
\mathrm{SrTi}_{1-\mathrm{X}} \mathrm{Mn}_{\mathrm{X}} \mathrm{O}_{3}\end{array}$ & Toluene & $\begin{array}{l}\mathrm{T}_{50}: 343^{\circ} \mathrm{C} \\
\mathrm{T}_{90}: 398^{\circ} \mathrm{C} \\
\mathrm{T}_{50}: 302^{\circ} \mathrm{C} \\
\mathrm{T}_{90}: 335^{\circ} \mathrm{C}\end{array}$ & - & $\begin{array}{l}\text { Incorporation of Mn attributed a } \\
\text { higher amount of oxygen vacancies in } \\
\text { the perovskite surface to promote the } \\
\text { toluene conversion. Supra facial } \\
\text { mechanism. }\end{array}$ \\
\hline [72] & $\begin{array}{c}\mathrm{LaMn}_{1-\mathrm{x}} \mathrm{B}_{\mathrm{x}} \mathrm{O}_{3} \\
(\mathrm{~B}=\mathrm{Co}, \mathrm{Ni}, \mathrm{Cu}, \mathrm{Al})\end{array}$ & $\begin{array}{c}\text { Toluene } \\
\text { LMLi } \\
\text { LMNi } \\
\text { LMCo } \\
\text { LMAl } \\
\\
\text { LMLi } \\
\text { LMNi } \\
\text { LMCo } \\
\text { LMAl }\end{array}$ & $\begin{array}{c}\mathrm{T}_{50}: \\
250^{\circ} \mathrm{C} \\
234^{\circ} \mathrm{C} \\
226{ }^{\circ} \mathrm{C} \\
242{ }^{\circ} \mathrm{C} \\
\mathrm{T}_{90}: \\
\text { none } \\
278^{\circ} \mathrm{C} \\
271^{\circ} \mathrm{C} \\
318^{\circ} \mathrm{C}\end{array}$ & - & $\begin{array}{l}\text { Addition of nickel into } \mathrm{LaMnO}_{3} \text { can } \\
\text { improve the catalytic oxidation of } \\
\text { toluene by generation of more } \mathrm{Mn}^{4+} \\
\text { species and oxygen vacancies and the } \\
\text { enhancing reducibility at low } \\
\text { temperature. }\end{array}$ \\
\hline [73] & $\mathrm{LaMnO} 3 / \delta-\mathrm{MnO} 2$ & Toluene & $\mathrm{T}_{90}: 258^{\circ} \mathrm{C}$ & - & - \\
\hline
\end{tabular}


Table 7. Cont.

\begin{tabular}{|c|c|c|c|c|c|}
\hline Refer & Catalyst & VOCs & Catalytic Performance & Remarks & Catalytic Mechanism \\
\hline [74] & $\mathrm{LaMnO}_{3}$ & Toluene & $\begin{array}{l}\mathrm{T}_{50}: 258^{\circ} \mathrm{C} \\
\mathrm{T}_{90}: 275^{\circ} \mathrm{C}\end{array}$ & - & - \\
\hline [85] & $\mathrm{CeO}_{2} @ \mathrm{SiO}_{2}-400$ & DCE & $\begin{array}{l}\mathrm{T}_{50}: 219^{\circ} \mathrm{C} \\
\mathrm{T}_{90}: 275^{\circ} \mathrm{C}\end{array}$ & $\begin{array}{l}\text { The conversion of } 1 \text { vol } \% \mathrm{H}_{2} \mathrm{O} \text { and } 3 \\
\text { vol } \% \mathrm{H}_{2} \mathrm{O} \text { decreased by } 6 \% \text { and } 19 \% \text {, } \\
\text { respectively. }\end{array}$ & $\begin{array}{c}\text { DCE }+ \text { acid sites } \rightarrow \mathrm{HCl}+\mathrm{VC} .(1) \\
\mathrm{VC} \rightarrow \text { reactive carbonium } \\
\text { ion } \rightarrow \text { adsorbed alcohol } \\
\text { species } \rightarrow \text { acetate species. Or }(2) \\
\text { VC } \rightarrow 1,1,2 \text {-trichloroethane } \rightarrow \\
\text { dichloroethylene formed (subsequent } \\
\text { chlorination reactions) } \rightarrow \mathrm{H}_{2} \mathrm{O}, \mathrm{CO}_{\mathrm{x}} \\
\text { and } \mathrm{HCl} .\end{array}$ \\
\hline [94] & $\mathrm{LaCoO}_{3}$ & Propane & $\begin{array}{l}\mathrm{T}_{50}: 208^{\circ} \mathrm{C} \\
\mathrm{T}_{90}: 238^{\circ} \mathrm{C}\end{array}$ & - & MVK mechanism. \\
\hline [97] & $\begin{array}{c}\mathrm{LaMnO}_{3} \text { and } \\
\mathrm{LaCoO}_{3}\end{array}$ & Chlorinated VOCs & - & - & 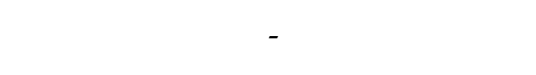 \\
\hline [104] & $\begin{array}{l}\mathrm{HCl} \text { modified } \\
\mathrm{La}_{0.5} \mathrm{Sr}_{0.5} \mathrm{MnO}_{3}\end{array}$ & Vinyl chloride & $\mathrm{T}_{100}: 300^{\circ} \mathrm{C}$ & - & $\begin{array}{l}\text { Doping and acid treatment obviously } \\
\text { promote the active } \mathrm{Mn}^{4+} \text { species } \\
\text { amount and oxygen activation ability, } \\
\text { and affect the chloric byproduct } \\
\text { distribution. The lower temperature } \\
\text { inhibits the Deacon reaction and } \\
\text { chlorination. }\end{array}$ \\
\hline
\end{tabular}




\subsubsection{Concentrated Oxidation Catalysts}

The low concentration of VOCs can limit the catalytic efficiency of catalysts. Some porous materials were investigated to the concentration and decomposition of VOCs, such as zeolite, $\gamma-\mathrm{Al}_{2} \mathrm{O}_{3}$ (Table 8 , Figure 13) $[84,101,173-175]$. R. Beauchet et al. tried to decompose the isopropanol and o-xylene mixture over the CsX, NaX, and HY zeolite [173]. O-xylene and isopropanol were totally decomposed at $250^{\circ} \mathrm{C}$ over the $\mathrm{NaX}$ zeolite. The addition of Pt on the zeolite can significantly increase the catalytic activity. The main obstacle for the application of zeolite was the coke that formed during the reaction, which led to the short lifetime. Amir Ikhlaq et al. studied the mechanism and kinetics of decomposition of chlorinated VOCs by ozonation over the $\gamma-\mathrm{Al}_{2} \mathrm{O}_{3}$ and ZSM-5 zeolite [174]. The results suggest that ozone reacts with the absorbed VOCs on the surface of catalysts. However, the ozonation process will increase the cost in most of the industrial processes. Yuexin Peng et al. supported $\mathrm{MnO}_{2}$ on the Al-rich $\beta$-zeolite to degrade toluene [75]. The $\mathrm{T}_{90}$ is $285^{\circ} \mathrm{C}$, which is much lower than the $\mathrm{MnO}_{2}$ supported on $\gamma-\mathrm{Al}_{2} \mathrm{O}_{3}$ due to the lattice oxygen species in $\mathrm{MnO}_{2}$ and absorbed oxygen species on the zeolite. $\mathrm{Cu}$ and $\mathrm{Co}$ were also used to modify the $\beta$-zeolite for the destruction of toluene and trichloroethylene [76]. The modified zeolite showed good stability in the reaction. The catalytic activity is mainly from the supported metal oxides. The suitable acidity and strong oxidation stability can improve the $\mathrm{CO}_{2}$ selectivity. Dedong He et al. modified the HZSM-5 zeolite with a series of rare earth elements, including $\mathrm{La}, \mathrm{Ce}, \mathrm{Pr}, \mathrm{Nd}, \mathrm{Sm}, \mathrm{Y}$, and $\mathrm{Er}$, for the catalytic decomposition of $\mathrm{CH}_{3} \mathrm{SH}$ [91]. $\mathrm{Cu}$ was also used to modify ZSM- 5 for the combustion of acrylonitrile. The isolated $\mathrm{Cu}$ is the active center. The $\mathrm{SiO}_{2} / \mathrm{Al}_{2} \mathrm{O}_{3}$ ratio can affect the ion exchange capacity and the catalytic performance of the catalyst. When the ratio is 26 , the catalyst shows the best catalytic activity [92]. SBA-15 was used to support $\mathrm{MnO}_{x}$ for the combustion of toluene and showed good catalytic activity [176]. The La modified HZSM-5 zeolite showed much better activity and stability than HZSM-5 due to the tunable acidity, which can promote the adsorption and activation of the $\mathrm{CH}_{3} \mathrm{SH}$ molecule and inhibit the formation of coke deposit. The previous researches showed the potential application in the industry. However, to fulfill the requirements of the industry, the concentration-catalysis process to remove VOCs needs more research on lifetime, catalytic efficiency.

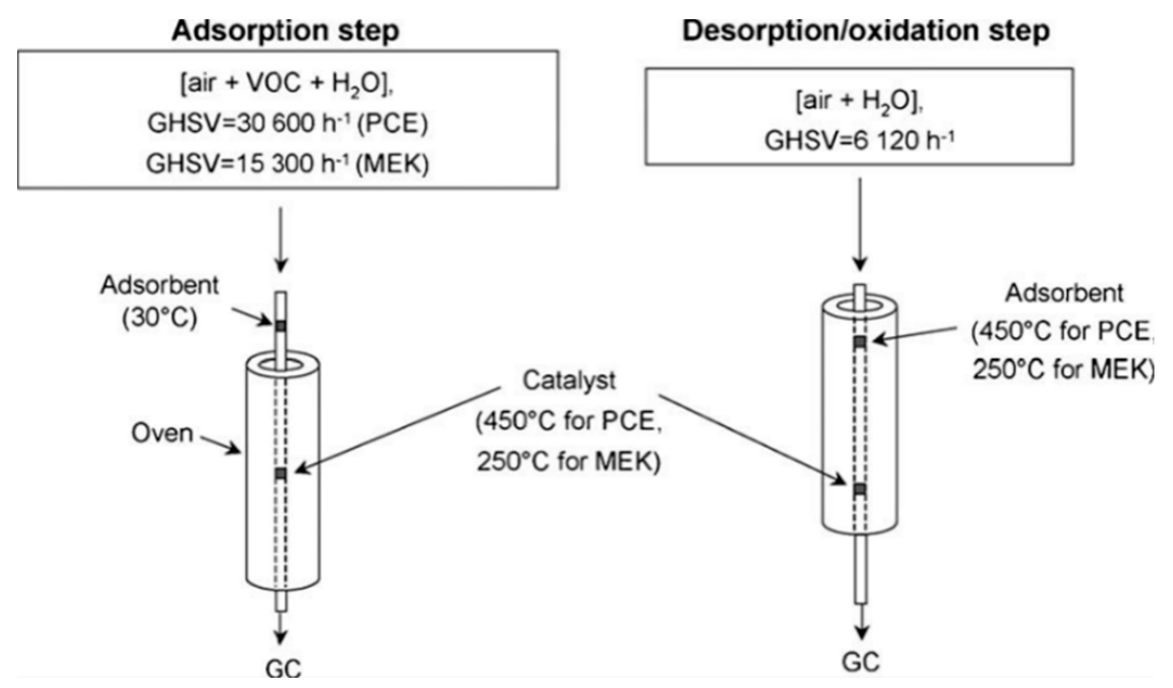

Figure 13. Scheme of adsorption/oxidation apparatus. Reprinted with permission from [1], 2009, Elsevier Ltd. 
Table 8. Concentrated oxidation catalysts for catalytic combustion of VOCs.

\begin{tabular}{|c|c|c|c|c|c|}
\hline Refer & Catalyst & VOCs & Catalytic Performance & Remarks & Catalytic Mechanism \\
\hline$[75]$ & $\begin{array}{c}\mathrm{MnO}_{\mathrm{x}} / \mathrm{H}-\text { Beta-SDS } \\
\mathrm{MnO}_{\mathbf{x}} / \mathrm{K}-\text { Beta-SDS } \\
\mathrm{MnOx} / \text { Si-Beta }\end{array}$ & Toluene & $\begin{array}{l}\mathrm{T}_{50}: 253,262,280^{\circ} \mathrm{C} \\
\mathrm{T}_{90}: 285,295,312{ }^{\circ} \mathrm{C}\end{array}$ & - & $\begin{array}{c}\text { Mars-Van Krevelen mechanism. Organic } \\
\text { molecules+lattice oxygen } \rightarrow \text { oxygen } \\
\text { vacancy }+\mathrm{CO}_{2}+\mathrm{H}_{2} \mathrm{O} \text {, oxygen } \\
\text { vacancy }+\mathrm{O}_{2} \rightarrow \text { lattice oxygen. }\end{array}$ \\
\hline$[84]$ & H-ZSM-5 and Na-ZSM-5 & $\begin{array}{l}\text { Cumene } \\
\text { Dichlorobenzene } \\
\text { Trichloro benzene }\end{array}$ & - & - & - \\
\hline [91] & La(13)/HZSM-5 & $\mathrm{CH}_{3} \mathrm{SH}$ & $\mathrm{T}_{100}: 40^{\circ} \mathrm{C}$ & - & $\begin{array}{c}\text { The La-modified HZSM- } 5 \text { increased basic } \\
\text { sites and displayed better adsorption } \\
\text { ability to } \mathrm{CH}_{3} \mathrm{SH} \text {, decreased in strong acid } \\
\text { sites and suppressed the formation of } \\
\text { coke deposit. }\end{array}$ \\
\hline [92] & $\mathrm{Cu}-\mathrm{ZSM}-5\left(\mathrm{SiO}_{2} / \mathrm{Al}_{2} \mathrm{O}_{3}=26\right)$ & $\mathrm{C}_{2} \mathrm{H}_{3} \mathrm{CN}$ & $\mathrm{T}_{90}: 325^{\circ} \mathrm{C}$ & $\begin{array}{l}\text { The catalyst has good } \\
\text { resistance to steam } \\
\text { poisoning. }\end{array}$ & $\begin{array}{c}\text { Without } \mathrm{H}_{2} \mathrm{O}: \\
\mathrm{C}_{2} \mathrm{H}_{3} \mathrm{CN}-\mathrm{SCC}+\mathrm{O}_{2} \rightarrow \mathrm{NCO} \rightarrow \text { oxidation } \\
\text { products; with } \mathrm{H}_{2} \mathrm{O}: \mathrm{C}_{2} \mathrm{H}_{3} \mathrm{CN}-\mathrm{SCC}+\mathrm{O}_{2}+ \\
\mathrm{H}_{2} \mathrm{O} \rightarrow \mathrm{NH}_{3} \rightarrow \text { products. }\end{array}$ \\
\hline [101] & $\mathrm{CsX}, \mathrm{NaX}$, and $\mathrm{HY}$ & VOCs mixture & $\mathrm{T}_{100}: 200^{\circ} \mathrm{C}$ & - & $\begin{array}{l}\text { Mars-Van Krevelen mechanism, involving } \\
\text { several redox steps. }\end{array}$ \\
\hline [176] & Mn/R-SBA-15 & Toluene & $\mathrm{T}_{98}: 240^{\circ} \mathrm{C}$ & - & $\begin{array}{c}\text { MVK mechanism. } \\
\mathrm{Mn}_{2} \mathrm{O}_{3}-\mathrm{MnO}_{2} / \mathrm{R}-\mathrm{SBA}-15 \text { supply more } \\
\text { lattice oxygen species. }\end{array}$ \\
\hline
\end{tabular}




\subsubsection{The Influence Factors on Catalytic Performance}

In the real industrial process, a lot of factors influence the catalytic efficiency. Firstly, the kind of VOCs determines the selection of catalysts. For example, noble metal catalysts show the best VOCs eliminating efficiency, but they are not suitable for the destruction of chlorine and sulfur containing VOCs due to the poison of the catalysts. Secondly, the surface area of the catalysts is the main factor that influences the catalytic activity. Research shows that $\mathrm{MnO}_{2}$ with a higher surface area showed much better catalytic performance than the one with a lower surface area [133]. Thirdly, the crystal type of catalysts also influences the catalytic performance of the catalysts with the same content. For instance, the catalytic performance of $\mathrm{TiO}_{2}$ with different crystal types, namely rutile and anatase, showed a different catalytic activity [121,167]. The humidity is the common content in the industrial waste gas. In most of the reports, water molecules can suppress the catalytic activity due to the complete adsorption on active sites and destruction of catalysts [177]. In other researches, humidity plays a positive role in the oxidation. In the catalytic combustion of chlorobenzene over $\mathrm{VO}_{\mathrm{x}} / \mathrm{TiO}_{2}$, $\mathrm{VO}_{\mathrm{x}}-\mathrm{WO}_{\mathrm{x}} / \mathrm{TiO}_{2}$, and $\mathrm{VO}_{\mathrm{x}}-\mathrm{MoO}_{\mathrm{x}} / \mathrm{TiO}_{2}$, water can remove the adsorbed $\mathrm{Cl}^{-}$from the catalysts surface and react with chlorine to produce $\mathrm{HCl}$ [134]. The water vapor can show a different effect on the same VOCs over different catalysts. Kullavanijayam et al. reported that water enhanced the catalytic oxidation of cyclohexene over the ceria-alumina supported $\mathrm{Pt}$ and $\mathrm{Rh}$, but it had a negative effect on the oxidation of cyclohexane over the ceria-alumina supported Pd catalyst [178]. CO is another poison for precious metal catalysts, so during the catalytic combustion, enough air is needed to avoid the generation of $\mathrm{CO}$. The life of catalysts is also important for catalytic combustion. The main obstacle for the development of catalysts for the catalytic combustion of halogen and sulfur containing VOCs is the short life of common catalysts. Although $\mathrm{V}$ oxides show less catalytic efficiency than other catalysts, it is still used in the elimination of halogen and sulfur containing VOCs due to its long catalytic life [152]. Since after the installation of catalytic combustion equipment, it will be in operation for a long time, therefore, the life of the catalysts determines the cost of catalytic combustion.

The catalytic combustion process has been well developed in recent years. The diversity of the catalysts has been investigated. However, there are still some obstacles on the way to the industrial application, such as short lifetime, high cost, and no universality to different kinds of VOCs. More work should be done on these problems.

\subsection{Photocatalytic Oxidation}

Photocatalytic reactions have drawn a lot of attention and have been well developed in recent years since Fujishima found the splitting water to $\mathrm{H}_{2}$ and $\mathrm{O}_{2}$ over $\mathrm{TiO}_{2}[55,179,180]$. Different kinds of photocatalysts have been developed to treat VOCs containing waste water, such as $\mathrm{TiO}_{2}, \mathrm{WO}_{3}, \mathrm{ZnO}_{2}$, $\mathrm{CdS}, \mathrm{g}-\mathrm{C}_{3} \mathrm{~N}_{4}$, and $\mathrm{BiOBr}$ (Table 9) [56,180-185]. The mechanism of photocatalysis is that when the light with a suitable wavelength radio on catalysts (semiconductors), the electrons and holes were separated and generated on the surface of catalysts, then the radicals of $\bullet \mathrm{OH}$ and $\mathrm{O}^{2-}$ was formed on the surface of catalysts, the VOCs reacted with these radicals and decomposed to $\mathrm{CO}_{2}$ and $\mathrm{H}_{2} \mathrm{O}$ at last $[186,187]$. In the water solution, water can react with the catalysts and form $\bullet \mathrm{OH}$, which is positive for the decomposition of VOCs in water. The photocatalytic elimination of VOCs in the gas phase follows a similar mechanism, but the radicals are main $\mathrm{O}^{2-}$ due to the shortage of humidity. In this section, the progress in the elimination of VOCs in the gas phase by photocatalytic methods was mainly discussed.

The most studied photocatalyst for the elimination of $\mathrm{VOCs}$ is $\mathrm{TiO}_{2}$ [44]. Wilson F. Jariam et al. degraded 17 kinds of VOCs with the concentration range of 400-600 ppmv over $\mathrm{TiO}_{2}$ under the radiation of ultraviolet light [98]. The results showed that trichloroethylene (99.9\%), isooctane (98.9\%), acetone (98.5\%), methanol (97.9\%), methyl ethyl ketone (97.1\%), t-butyl methyl ether $(96.1 \%)$, dimethoxymethane (93.9\%), methylene chloride (90.4\%), methyl isopropyl ketone $(88.5 \%)$, isopropanol $(79.7 \%)$, chloroform $(69.5 \%)$, and tetrachloroethylene $(66.6 \%)$ were decomposed efficiently over $\mathrm{TiO}_{2}$. The photodegradation of isopropylbenlene (30.3\%), methyl chloroform $(20.5 \%)$, and pyridine $(15.8 \%)$ 
on $\mathrm{TiO}_{2}$ was not as efficient as other VOCs. The catalytic lifetime was also tested by toluene. The conversion of toluene decreased to $20.9 \%$ after a 150 min test, but the deactivated catalysts can be easily regenerated by washing with $\mathrm{H}_{2} \mathrm{O}_{2}$ and illumination. F. B. Li et al. prepared La ion doped $\mathrm{TiO}_{2}$ by the sol-gel method for photodegradation of benzene, toluene, ethylbenzene, and o-xylene in the gas phase [188]. The results showed that the $\mathrm{La}$ ion doped $\mathrm{TiO}_{2}$ performed much better than the pure $\mathrm{TiO}_{2}$. This was due to the improved adsorption ability and the enhanced electron-hole pairs separation by the presence of $\mathrm{Ti}^{3+}$ and the electron transfer between the conduction band/defect level and lanthanide crystal field state. Tânia M. Fujimoto et al. supported the palladium on $\mathrm{TiO}_{2}$ for the photocatalytic decomposition of octane, isooctane, n-hexane, and cyclohexane in a low concentration (100 120 ppmv) [99]. The modified catalysts showed excellent catalytic activity in the decomposition of VOCs rapidly. V. Héquet et al. used a closed-loop reactor to study the mixture effect over the $\mathrm{P}_{25} \mathrm{TiO}_{2} / \mathrm{SiO}_{2}$ mixture (Figure 14) [189]. They have developed the accurate analytical methods to identify and quantify the majority of the potential formed intermediates, which provide an efficient way to study the reaction mechanism. Yajie Shu et al. used $\mathrm{Mn}$ doped $\mathrm{TiO}_{2}$ to degrade benzene by $\mathrm{O}_{3}$ under vacuum ultraviolet (VUV) irradiation [62]. The doped $\mathrm{TiO}_{2}$ showed better performance than the undoped one and $\mathrm{P}_{25}$ due to the formation of highly reactive oxidizing species. Jian Ji et al. showed that compared with the one without UV radiation, the UV radiation can improve the removal efficiency of benzene by about 10\% (Figure 15) [63]. Marta Stucchi et al. developed a simultaneous photodegradation system for the VOC mixture elimination by $\mathrm{TiO}_{2}$ powders, which showed a good efficiency [86]. Huiling Huang et al. developed Mn modified ZSM-5 as catalysts for VUV photolysis combined with ozone-assisted catalytic oxidation and studied the mechanism [102]. The catalysts showed good efficiency. Although most of the catalysts showed excellent activity in the elimination of VOCs under UV light, the light utilization efficiency was still low due to the low percent of the UV light in nature light. Xufang Qian et al. designed mesoporous $\mathrm{TiO}_{2}$ films coated with carbon foam for photodegradation of acetone and toluene, which can converse more than $90 \%$ of $\mathrm{VOCs}_{\text {so }} \mathrm{CO}_{2}$ under visible light due to the plausible carbon doping and the strong interaction between the $\mathrm{TiO}_{2}$ precursor and the hydro-carbon foams [100]. The graphite- $\mathrm{SiO}_{2}-\mathrm{TiO}_{2}$ composite and $\mathrm{BiOBr} @ \mathrm{SiO}_{2}$ flower-like nanospheres were also used for photodegradation of VOCs under visible light and showed good catalytic activity [64]. The low solar utilization ratio was the main obstacle to improve photocatalytic efficiency. In order to improve the efficiency, materials with a good light adsorption ability was applied. Yun-En Lee et al. prepared black- $\mathrm{TiO}_{2}$ and $\mathrm{LFO} /$ black- $\mathrm{TiO}_{2}$ and they showed excellent photo catalytic activity for the removal of toluene and IPA due to their good light adsorption ability [77]. The modification of $\mathrm{g}-\mathrm{C}_{3} \mathrm{~N}_{4}$ by hydroxyl groups can enhance visible light-driven photocatalytic properties of $g-C_{3} N_{4}$ obviously which can improve the adsorption energy of $g-C_{3} N_{4}$ for water and phenol [88]. The structure of catalysts was also carefully tuned to improve the catalytic activity [190]. Bettini $S$ et al. insulated a layer of $\mathrm{SiO}_{2}$ between zinc oxide and nano silver and controlled the thickness of the insulated layer, which enhanced the photocatalytic oxidation ability of the catalyst significantly [78]. The photocatalytic activity of $\mathrm{ZnO}$ can also be improved by doping $\mathrm{CuO}$ [191]. The photocatalytic elimination of VOCs is one of the most promising methods. However, the industrial application of this method is still a problem. 


\section{Photocatalytic unit}

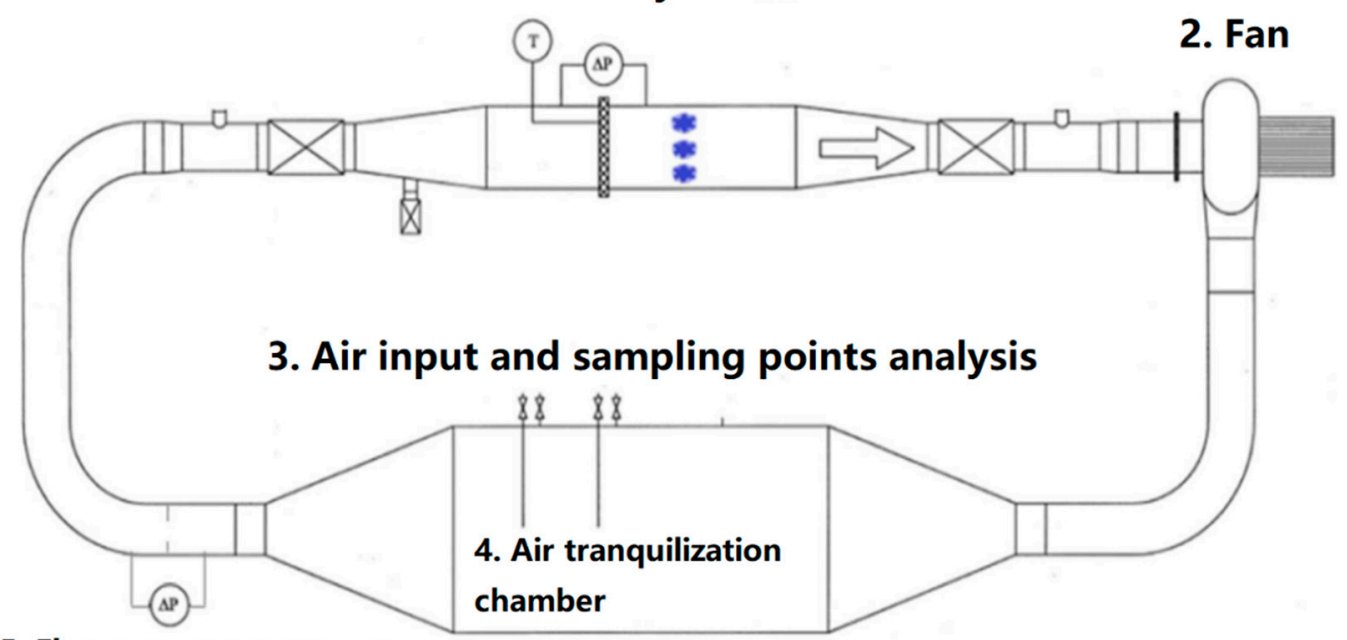

\section{Flow rate measurement}

Figure 14. A 420-L of continuous closed-loop photocatalytic reactor: (1) Photocatalytic unit containing the $\mathrm{TiO}_{2}$ photocatalytic medium and the ultraviolet (UV) lamps, (2) fan, (3) air input and sampling points for analysis, (4) air tranquilization chamber, (5) flow rate measurement. Reprinted with permission from [176], 2018, Elsevier Ltd.

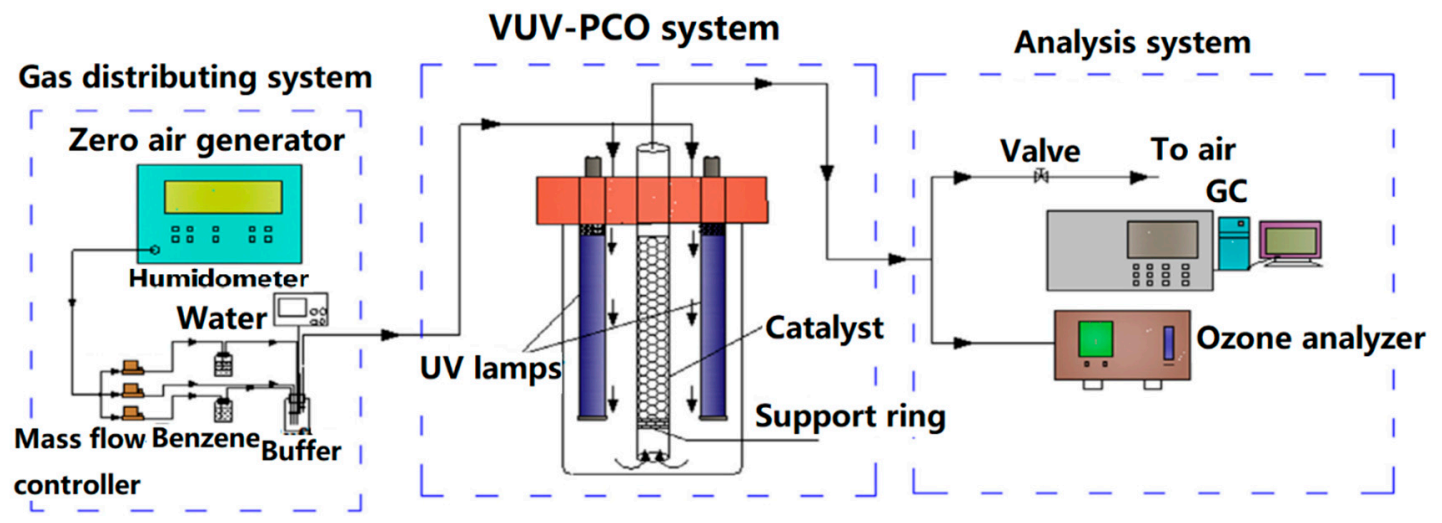

Figure 15. The schematic diagram of the VUV-PCO system. Reprinted with permission from [63], 2017, Elsevier Ltd.

There is a large amount of reports on the photocatalytic elimination of VOCs. The advantages of this process are green, energy saving, and clean. However, the reported catalysts are not efficient enough for an industrial application at the present stage. Firstly, the reaction needs more time than other methods. Secondly, most of the reported catalysts could not use visible light as energy to start the reactions, which limited the improvement of quantum efficiency, so it still needs to develop efficient photocatalysts and design effective reaction systems. 
Table 9. Photocatalysts for elimination of VOCs.

\begin{tabular}{|c|c|c|c|c|c|}
\hline Refer & Catalyst & VOCs & Catalytic Performance & Remarks & Catalytic Mechanism \\
\hline [63] & Mesoporous $\mathrm{TiO}_{2}$ & Benzene & - & - & $\begin{array}{c}\text { Benzene } \rightarrow \text { (splitting of benzene, } \\
\text { hydrogenation, } \mathrm{H} \text { abstraction by OH radicals) } \\
\text { six-member ring cyclitols+aldehydes ketones, } \\
\text { etc. } \rightarrow \text { a class of fulvene (isomerization) } \rightarrow \\
\qquad \mathrm{CO}_{2}+\mathrm{H}_{2} \mathrm{O} \text {. } \\
\text { Benzene is mainly degraded by } \\
\text { photo-generated electron-hole pairs and } \\
\text { hydroxyl radicals. }\end{array}$ \\
\hline [64] & Graphite- $\mathrm{SiO}_{2}-\mathrm{TiO}_{2}$ & Benzene & $\begin{array}{l}\text { The graphite- } \mathrm{SiO}_{2}-\mathrm{TiO}_{2} \text { composites } \\
\text { exhibited higher photocatalytic } \\
\text { activity for degradation of benzene } \\
\text { gas under visible light irradiation } \\
\text { than that of pure } \mathrm{TiO}_{2} \text {. }\end{array}$ & - & $\begin{array}{l}\text { Optimum concentration of graphite facilitates } \\
\text { the separation of photogenerated } \\
\text { electron-hole pairs for graphite- } \mathrm{SiO}_{2}-\mathrm{TiO}_{2} \\
\text { composites by visible light. }\end{array}$ \\
\hline [77] & $\mathrm{LaFeO}_{3} /$ black-TiO 2 & Toluene & $\begin{array}{l}\text { The removal efficiency of black- } \mathrm{TiO}_{2} \\
\text { and } \mathrm{LaFeO}_{3} \text { for toluene was } 89 \% \\
\text { and } 98 \% \text {, respectively, and the } \\
\text { removal efficiency for IPA was } 90 \% \\
\text { and } 94 \% \text {, respectively. }\end{array}$ & - & $\begin{array}{l}\text { Cat.+photon (the wavelength shorter than } 440 \\
\mathrm{~nm}) \rightarrow \text { electrons }+\mathrm{O}_{2} \rightarrow \mathrm{O}^{2-}, \mathrm{O}^{2-}+\text { toluene and } \\
\qquad \mathrm{IPA} \rightarrow \mathrm{CO}_{2}+\mathrm{H}_{2} \mathrm{O}_{(\mathrm{g})} \text {. }\end{array}$ \\
\hline [78] & $\mathrm{Pd} / \mathrm{CeO}_{2} / \gamma-\mathrm{Al}_{2} \mathrm{O}_{3}$ & $\begin{array}{c}\text { Toluene } \\
\text { Acetone } \\
\text { Ethyl acetate }\end{array}$ & $\begin{array}{l}\mathrm{T}_{98}: \\
205^{\circ} \mathrm{C} \\
220^{\circ} \mathrm{C} \\
275^{\circ} \mathrm{C}\end{array}$ & - & - \\
\hline [79] & $\begin{array}{l}\mathrm{CuO} / \mathrm{ZnO} \text { nanocomposite } \\
\text { photocatalysts }\end{array}$ & $\begin{array}{l}\text { Toluene } \\
\text { Iopropanol }\end{array}$ & 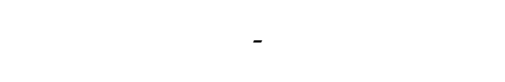 & - & $\begin{array}{c}\text { Photooxidative activity and stability over } \mathrm{ZnO} \\
\text { are improved by loading } \mathrm{CuO} .\end{array}$ \\
\hline [86] & $\mathrm{TiO}_{2}$ & $\begin{array}{l}\text { Formaldehyde } \\
\text { acetaldehyde }\end{array}$ & $\begin{array}{c}\mathrm{TiO}_{2} \text { degraded almost } 100 \% \text { of } \\
\text { formaldehyde or acetaldehyde at a } \\
\text { starting concentration of } 400-500 \\
\text { ppb with a relative humidity of } 40 \% \text {. }\end{array}$ & - & $\begin{array}{c}\text { The rate-determining step is the adsorption } \\
\text { (external diffusion) on the catalysts active } \\
\text { sites, thus the higher the surface area, the } \\
\text { higher the degradation. } \\
\text { OH }\end{array}$ \\
\hline [88] & $\mathrm{g}-\mathrm{C}_{3} \mathrm{~N}_{4}$ & $\begin{array}{l}\text { Benzoquinone } \\
\text { Hydroquinone catechol }\end{array}$ & - & - & $\begin{array}{c}\text { radicals }+ \text { phenol } \rightarrow \text { dihydroxycyclohexadienyl } \\
\text { radical adducts } \rightarrow \text { phenoxy radicals } \rightarrow \mathrm{H}_{2} \mathrm{O}(\mathrm{a} \\
\text { very slow process), adducts }+ \text { dissolved } \\
\mathrm{O}_{2} \rightarrow \text { dihydroxy intermediates }\left(-\mathrm{HO}_{2}\right) \\
\rightarrow \mathrm{CO}_{2}+\mathrm{H}_{2} \mathrm{O}\end{array}$ \\
\hline
\end{tabular}


Table 9. Cont

\begin{tabular}{|c|c|c|c|c|c|}
\hline Refer & Catalyst & VOCs & Catalytic Performance & Remarks & Catalytic Mechanism \\
\hline [98] & $\mathrm{La}^{3+}-\mathrm{TiO}_{2}$ and $\mathrm{Nd}^{3+}-\mathrm{TiO}_{2}$ & VOCs mixture & $\begin{array}{l}1.2 \% \mathrm{La}^{3+}-\mathrm{TiO}_{2} \text { had the highest } \\
\text { photocatalytic activity. }\end{array}$ & - & - \\
\hline [99] & $\mathrm{TiO}_{2} / \mathrm{Pd}$ & VOCs & $\begin{array}{c}\text { The conversion rate of VOCs } \\
\text { reached } 90 \% \text { when the residence } \\
\text { time was } 27 \mathrm{~s} .\end{array}$ & - & - \\
\hline [102] & Mn/ZSM-5 & $\mathrm{O}_{3}$ & $\begin{array}{c}\mathrm{O}_{3} \text { can be efficiently decomposed by } \\
\text { the Mn/ZSM- } 5 \text { and used for benzene } \\
\text { degradation through the OZCO. }\end{array}$ & - & $\begin{aligned} \text { Benzene }+\bullet \mathrm{OH} & \rightarrow \text { phenol } \rightarrow \text { benzoquinone } \\
& \rightarrow \mathrm{CO}_{2}+\mathrm{H}_{2} \mathrm{O}\end{aligned}$ \\
\hline$[100]$ & Meso-TiO 2 /hydro-CF & VOCs & - & - & $\begin{array}{l}\text { Promotion effects on degradation of gaseous } \\
\text { polar acetone come from well crystallized } \\
\text { anatase nanocrystals, hydro-CF skeleton for } \\
\text { adsorption, and fast mass transportation } \\
\text { within the hierarchical frameworks. }\end{array}$ \\
\hline
\end{tabular}




\subsection{Non-Thermal Plasma Process}

In the non-thermal plasma process, electrons and their surroundings are not in a thermal equilibrium, so the electrons are heated by electric discharges instead of the gas itself, and produce the electrons with high energy, active radicals and ions which can promote numerous chemical reactions in the ionized zones. It can be used to treat the high flow for both low ( $<100 \mathrm{ppmv})$ and high (>1000 ppmv) concentrations of VOCs, including toluene, benzene, acetone, trichloroethylene, etc. (Table 10) [47]. The main bottlenecks for the commercialization of the technology are the formation of poison byproducts and high energy consumption. The discharge methods are important for the VOCs removal efficiency, which includes corona discharge, surface discharge, microwave discharge, dielectric barrier discharge, and packed bed dielectric barrier discharge [190-194]. Among these discharge types, the packed bed dielectric barrier discharge shows the most potential in the industry application [195]. Savita K. P. Veerapandian et al. reviewed the packed bed DBD [47]. The influences of dielectric constant, packing materials size, shape, surface properties, and the byproducts formation were discussed.

Using porous and catalytic materials as the packed bed can increase the resistant time of VOCs and decrease the unwanted byproducts, such as $\mathrm{O}_{3}, \mathrm{NO}_{x}$, and $\mathrm{CO}$ (Figure 16) [196]. The packed bed materials can be non-catalytic porous materials, such as activated carbon, porous $\mathrm{Al}_{2} \mathrm{O}_{3}$, glass, zeolite, graphene oxide, and the catalysts, such as metal oxides, noble metal loaded metal oxides, and catalytic porous materials [197]. For example, using $\mathrm{Al}_{2} \mathrm{O}_{3}$ as the packed bed can concentrate VOCs molecules on its surface and weaken the bond energy of VOCs, which can enhance the dissociation when these adsorbed molecules encounter the active species in the plasma and increase the collision probability and deep oxidation of VOCs [198]. Lee et al. used porous $\gamma-\mathrm{Al}_{2} \mathrm{O}_{3}$ as the packed bed to oxidize toluene to $\mathrm{CO}_{2}$. It showed a $100 \%$ conversion of toluene and high $\mathrm{CO}_{2}$ selectivity [199]. Gandhi's research results showed that a large surface area and pore volume of alumina can not only increase the conversion of ethylene to $\mathrm{CO}_{2}$ or $\mathrm{CO}$, but also decrease the selectivity of unwanted byproducts, such as acetaldehyde, acetylene, methane, $\mathrm{N}_{2} \mathrm{O}$, and $\mathrm{O}_{3}$ [200]. Other researches also showed the same phenomenon in the abatement of benzene, acetone, formaldehyde, TCE, chlorobenzene in non-thermal plasma with the packed bed which has a large surface area and pore volume, such as porous alumina, $\mathrm{TiO}_{2}$, zeolite, and porous metal oxides [199,201-203]. Due to the large surface area, the retention time and concentration of VOCs molecules increased which led to the increase of collisions between VOCs and active species [200], the adsorption effect can weaken the chemical bond of VOCs [204], and form micro-discharges in the micro-pores in addition to the micro-discharges in the gas phase.

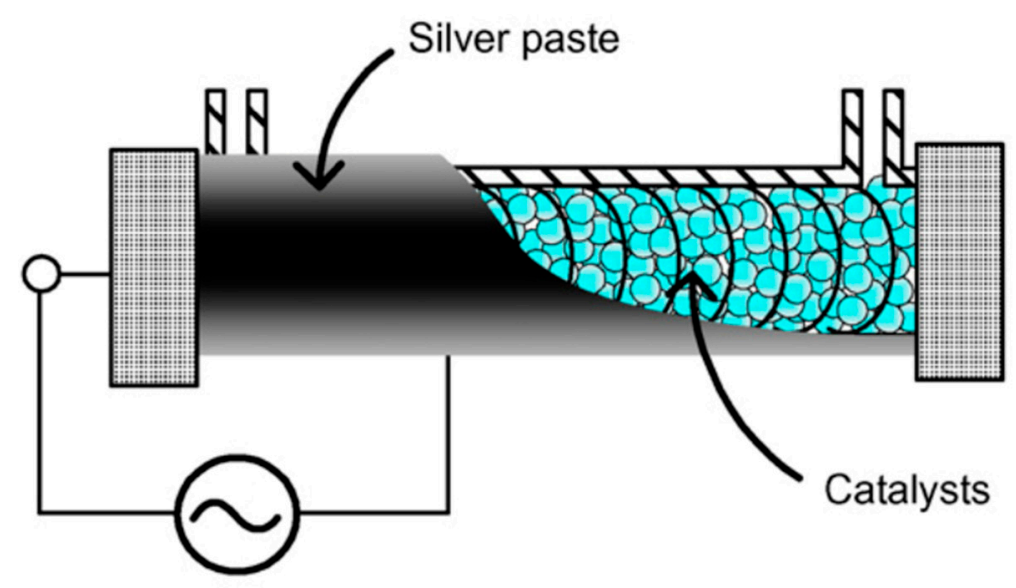

Figure 16. Schematic diagram of the plasma-driven catalyst (PDC) reactor. Reprinted with permission from [196], 2007, Elsevier Ltd.

The addition of catalysts in the non-thermal plasma process as the packed bed can enhance the VOCs removal efficiency significantly due to the plasma assisted reactions on the surface of the catalyst. 
These plasma-assisted reactions can generate some active radical species, such as intermediates with electrons, $\mathrm{O} \cdot \mathrm{OH} \cdot \mathrm{N}_{2} \cdot \mathrm{NO} \cdot, \mathrm{N}_{2} \mathrm{O} \cdot$ in the gas phase and $\mathrm{O} \cdot, \mathrm{OH} \cdot$ on the surface of catalysts, which can improve the VOCs removal efficiency and increase the $\mathrm{CO}_{\mathrm{x}}$ selectivity [80]. The catalysts include metal oxides, such as $\mathrm{CuO}, \mathrm{MnO}_{2}, \mathrm{CeO}_{2}, \mathrm{NiO}, \mathrm{CoO}_{2}$, and $\mathrm{Fe}_{2} \mathrm{O}_{3}$, and noble metals, such as $\mathrm{Pt}, \mathrm{Au}$, and $\mathrm{Ag}[65,196,203,205-209]$. The different kinds of oxygen species in catalysts, namely lattice oxygen, surface oxygen, and absorbed oxygen, are very helpful for the oxidation of VOCs, which can activate the oxygen molecules in the gas phase. P.J. Asilevi et al. established a laboratory scale DBD reactor for the removal of toluene by $\cdot \mathrm{O}$ and $\cdot \mathrm{OH}$ which were generated from the reaction between $\mathrm{O}_{2}$ and $\mathrm{H}_{2} \mathrm{O}$ and the removal efficiency of VOCs can be improved by increasing the oxygen concentration and relative humidity [210]. Lu et al. used $\mathrm{FeO}_{x} / \mathrm{SBA}-15$ as the packed bed to eliminate toluene in the non-thermal plasma process. The results showed that the presence of $\mathrm{Fe}^{2+}$ can increase the toluene removal efficiency and $\mathrm{CO}_{\mathrm{x}}$ selectivity obviously and reduce the formation of unwanted and toxic byproducts [80]. Zhu et al. prepared $5 \mathrm{wt} \% \mathrm{CuO} /-\mathrm{Al}_{2} \mathrm{O}_{3}$ as the packed bed to the abatement acetone. It showed better performance than the one without $\mathrm{CuO}$ due to the better reducibility and abundant active oxygen species [87]. Li et al. prepared $\mathrm{Pt} / \mathrm{Al}_{2} \mathrm{O}_{3}$ and it showed higher decomposition efficiency than the unloaded $\mathrm{Al}_{2} \mathrm{O}_{3}$ due to the presence of $\mathrm{Pt}$, which increases the number of active sites and reduces the activation energy of the decomposition reaction and suppresses harmful $\mathrm{NO}_{x}$ formation [65]. The research by Hua Song et al. showed that $\mathrm{CoMnO}_{\mathrm{x}} / \mathrm{TiO}_{2}$ can obviously improve the plasma removal of VOCs [103].

There are other factors that influence the VOCs removal efficiency, such as humidity and the plasma structure. In industrial conditions, the humidity in the VOCs stream is the factor which must be considered. The humidity has both a positive and negative effect on the removal of VOCs in the non-thermal plasma process. The water molecules can modify the surface of the packed bed, especially the catalytic packed bed, quench the free electrons and active species, and produce $\mathrm{OH}$ radicals during the discharge process $[103,202,207,211,212]$. A lot of studies showed that the presence of humidity can reduce the VOCs removal efficiency by quenching the high energy electrons and reactive species, and modify the catalysts surface $\left(\mathrm{BaTiO}_{3}\right)$ in the decomposition of toluene, benzene, xylene, and $C_{2} F_{2}[103,207,212]$. On the other hand, the humidity can suppress the formation of toxic byproducts, such as CO and NOx by deactivation of some oxygen species in the catalysts $[213,214]$. The optimization of the plasma structure also can enhance the VOCs decomposition efficiency. Muhammad Farooq Mustafa et al. designed a double dielectric barrier discharge reactor, in which the conversion of tetrachloroethylene, toluene, trichloroethylene, benzene, ethyl acetate, and carbon disulfide can be $100 \%$ with $\mathrm{BaTiO}_{3}$ and HZSM-5 as catalysts, respectively [215].

There are also some disadvantages in the non-thermal plasma process, such as high energy consumption, low VOCs conversion (mostly about $30 \%$ to $70 \%$ ), low $\mathrm{CO}_{\mathrm{x}}$ selectivity, and produced unwanted byproducts. The other barrier for the application of non-thermal plasma in the industry is the high cost and the high energy consumption. The way to solve these problems is the development of a highly efficient catalytic packed bed and the optimization of an electrode structure. 
Table 10. Catalysts for VOCs elimination through the non-thermal plasma process.

\begin{tabular}{|c|c|c|c|c|c|}
\hline Refer & Catalyst & VOCs & Catalytic Performance & Remarks & Catalytic Mechanism \\
\hline$[65]$ & $\mathrm{Pt} / \mathrm{Al}_{2} \mathrm{O}_{3}$ & $\begin{array}{c}\text { Benzene } \\
\text { byproducts }\end{array}$ & - & - & - \\
\hline [80] & $\mathrm{FeO}_{\mathrm{x}} / \mathrm{SBA}-15$ & Toluene & - & $\begin{array}{l}\text { Under the condition of } 3 \% \mathrm{Fe} \\
\text { loading, the oxidation of toluene is } \\
\text { the best one. }\end{array}$ & 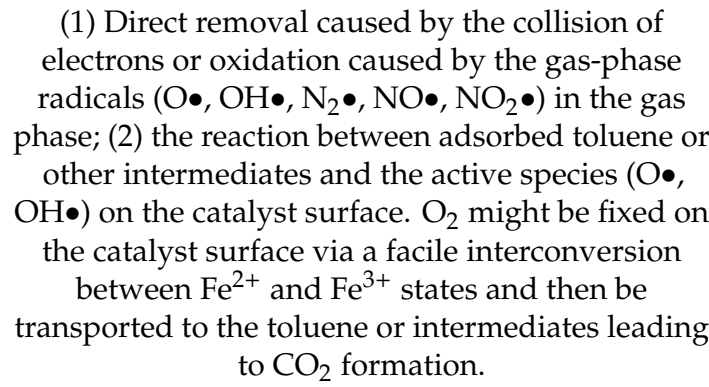 \\
\hline [87] & $\mathrm{CuO} / \mathrm{g}-\mathrm{Al}_{2} \mathrm{O}_{3}$ & Ccetone & - & $\begin{array}{c}5.0 \mathrm{wt} \% \mathrm{CuO} / \mathrm{g}-\mathrm{Al}_{2} \mathrm{O}_{3} \text { catalyst has } \\
\text { the highest removal rate of acetone, } \\
\text { reaching } 67.9 \% \text {. }\end{array}$ & $\begin{array}{l}\text { Both short-lived radicals and acetone/intermediates } \\
\text { can be adsorbed on the catalyst surfaces to initiate a } \\
\text { series of surface oxidation reactions, forming } \mathrm{CO} \text {, } \\
\qquad \mathrm{CO}_{2}, \mathrm{H}_{2} \mathrm{O} \text {, and byproducts. }\end{array}$ \\
\hline [103] & $\mathrm{CoMnO}_{\mathrm{x}} / \mathrm{TiO}_{2}$ & $\mathrm{O}_{3}$ & - & $\begin{array}{c}\text { When the temperature is } 320^{\circ} \mathrm{C} \text {, } \\
\text { the decomposition efficiency of } \mathrm{O}_{3} \\
\text { is } 98 \% \text {. }\end{array}$ & 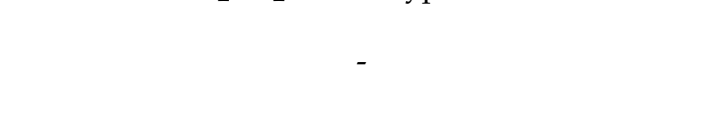 \\
\hline
\end{tabular}




\subsection{Electron Beam Treatment}

In the electron beam technology, an electron beam generated from an EB accelerator and absorbed by the background gas to ionize the air and form some active oxidizing radicals, such as $\cdot \mathrm{OH}$ and $\cdot \mathrm{HO}_{2}$ and excited ions as $\mathrm{O}\left({ }^{3} \mathrm{P}\right)$, which is critical for the destruction of VOCs [216-218]. The oxidizing radicals reacted with VOCs and decomposed them to inorganic compounds. There are three types of accelerators used for VOCs decomposition, including transformer accelerator, ultrahigh frequency accelerator, and linear microwave accelerator [217]. This technology has been used for the elimination of acetaldehyde, benzene, dodecane, ethylbenzene, hexadecane, pentane, styrene, tetradecane, toluene, trimethylamine, turpentine, xylene, dimethyl sulfide, dimethyl disulfide, methyl mercaptan, and chlorinated VOCs in a lab or pilot scale [218-228]. The electron beam can treat the waste gas with a low concentration of VOCs (10 to hundreds ppm). The initial electrons from an electron beam can hardly decompose the VOCs molecules, while it can react with background gases and form active radicals. The way to improve the abatement rate of aromatic VOCs includes the addition of humidity (formation of $\mathrm{OH} \cdot$ ), ammonia, chlorinated VOCs, ozone, and using an absorbed dose $[220,229]$. The reaction temperature, VOCs molecular structure, and background gas also influence the decomposition efficiency. However, there are also some drawbacks of the electron beam technology, the generation of active oxidizing radicals, e.g., $\cdot \mathrm{OH}$ and $\cdot \mathrm{HO}_{2}$ can lead to the creation of toxic intermediates, especially in the case of the VOCs emission with an unknown composition, such as the production of toxic byproducts (aerosol, ozone, $\mathrm{CO}$, and other trace organic compounds). The equipment is also too complex. It still needs more research for the industrial application.

\section{Outlook of the Different Kinds of Technologies}

Different kinds of VOCs were emitted from different parts of pharmaceutical production processes. With the new VOCs discharge standards coming into force, it is critical for the pharmaceutical companies to eliminate VOCs exhaustively in China. According to the production processes and conditions, the suitable VOCs elimination technologies must be selected with the highest efficiency and at the lowest cost. Adsorption, absorption, incineration, catalytic combustion, biodegradation, and non-thermal plasma technologies have been applied in factories for the abatement of VOCs. Adsorption and absorption are commonly the first used methods to recycle useful VOCs. Then, the rest of the VOCs were decomposed by other technologies, such as incineration, catalytic combustion, non-thermal plasma, biodegradation, and non-thermal plasma. The key point for the improvement of catalytic combustion and non-thermal plasma is the development of efficient catalysts or packed bed materials. Some new technologies are emerging for the abatement of VOCs, such as photocatalysis and electron beam radiation. These new technologies have shown good potential for the elimination of VOCs with a high efficiency and low energy cost. However, they still have some engineering problems for the industrial application. It is important to develop VOCs elimination technologies to decrease the amounts of VOCs in the discharged waste gas. The final solution for the VOCs is avoiding the emission of VOCs from the whole pharmaceutical production processes by developing new chemical and engineering technologies for the production of medicine.

Author Contributions: L.Z. has written the main content of this paper. C.M. has added some new research papers of this topic and summarized the catalysts used in different researches. J.H. has improved the English writing of the whole paper and written parts of photocatalysis parts. R.L. has written the parts of VOCs elimination policies in China and improve the structure of the paper. J.Y. has designed the whole structure of the paper and improved the manuscript a lot. All authors have read and agreed to the published version of the manuscript.

Funding: This research was funded by the Scientific Technology Research Program for the University in Hebei Province Youth Project (No. QN2020126).

Acknowledgments: The authors gratefully acknowledge the help of Jing Wang and Ping Chen from Tianjushi Engineering\&Technology Group Co., Ltd. who gave us a lot of suggestions about the VOCs treatment technologies and policies in China. The authors gratefully acknowledge the help of Rose Amal for the help of improvement the parts of catalytic processes for the treatment of VOCs. 
Conflicts of Interest: The authors declare no conflict of interest.

\section{References}

1. Li, W.B.; Wang, J.X.; Gong, H. Catalytic combustion of VOCs on non-noble metal catalysts. Catal. Today 2009, 148, 81-87. [CrossRef]

2. Muhammad, S.K.; Shaikh, A.R.; Mohammad, M.H. Catalytic oxidation of volatile organic compounds (VOCs): A review. Atmos. Environ. 2016, 140, 117-134.

3. Cheng, G. Study on VOCs Emissions Research Present Situation and the Emission Reduction Potential of Key Industries in Hebei Province. Master's Thesis, Hebei University of Science and Technology, Shijiangzhuang, China, 2016.

4. Li, Y. Study on Emission Standard of Air Pollutants for Pharmaceutical Industry Chemical Synthesis Products Category. Master's Thesis, Zhejiang University of Technology, Hangzhou, China, 2016.

5. Atkinson, R. Gas-phase tropospheric chemistry of volatile organic compounds: 1. Alkanes and alkenes. J. Phys. Chem. Ref. Data 1997, 26, 215-229. [CrossRef]

6. Fontane, H.; Veillerot, M.; Gallo, J.C.; Guillermo, R. 8th International Symposium on Transport and Air Pollution; Verlag der Technischen Universität Graz: Graz, Austria, 1999.

7. Rivière, E. CITEPA Report; Fieldwork Inhabitants: Paris, France, 1998.

8. Duncan, B.N.; Yoshida, Y.; Olson, J.R.; Sillman, S.; Martin, R.V.; Lamsal, L.; Hu, Y.; Pickering, K.E.; Retscher, C.; Allen, D.J.; et al. Application of OMI observations to a space-based indicator of NOx and VOC controls on surface ozone formation. Atmos. Environ. 2010, 44, 2213-2223. [CrossRef]

9. Atkinson, R. Atmospheric chemistry of VOCs and $\mathrm{NO}_{\mathrm{x}}$. Atmos. Environ. 2000, 34, 2063-2101.

10. National Bureau of Statistics of the People's Republic of China. Available online: http://www.stats.gov.cn/tjsj/ (accessed on 26 May 2020).

11. Chen, Y. Study on Current and Future Industrial Emission of Volatile Organic Compounds in China. Master's Thesis, South China University of Technology, Guangzhou, China, 2011.

12. Huang, W. Characteristics of Industrial VOCs Emissions and Evaluation of Control Technology in China. Master's Thesis, Zhejiang University, Hangzhou, China, 2016.

13. Ministry of Ecological Environment of the People's Republic of China. Standards for Emissions of Atmospheric Pollutants from Pharmaceutical Industry (Draft for Comments); Ministry of Ecological Environment of the People's Republic of China: Beijing, China, 2017.

14. Lu, Y. Establishment and Application on Assessment System of VOCs's Control Technology of Pharmaceutical Industry. Master's Thesis, Hebei University of Science and Technology, Shijiazhuang, China, 2016.

15. Hu, G. Research of Emission and Control Technology of VOCs and Odorous Gas from Pharmacy Industry in China. Master's Thesis, Nankai University, Tianjing, China, 2013.

16. Li, X. The System of Discharge Standards of Pollutants for Pharmaceutical Industry and a case Study. Master's Thesis, The Chinese Research Academy of Environmental Science, Beijing, China, 2006.

17. He, J. Fermentology; China Medical Science Press: Beijing, China, 2009.

18. Wang, Y. Chemical Pharmaceutical Technology; Chemical Industry Press: Beijing, China, 2008.

19. Zhou, T.; Ma, Q.; Chen, H. Treatment of chemical synthesized pharmaceutical wastewater by hybrid biological reactor. Ind. Water Wastewater 2010, 41, 42-45.

20. Chen, P. Pharmaceutical Technology; Hubei Science and Technology Press: Wuhan, China, 2008.

21. Qi, X. Modern Biopharmaceutical Technology; Chemical Industry Press: Beijing, China, 2009.

22. Cao, G. Pharmaceutical Engineering of Traditional Chinese Medicine; Chemical Industry Press: Beijing, China, 2004.

23. Wu, C. The thinking and Countermeasures for the Structuring of Learning Seals Team in Chengdu Zhonghui Pharmaceuticals Company. Master's Thesis, Southwestern University of Finance and Economics, Chengdu, China, 2006.

24. Chen, Y.; Ye, D.; Liu, X.; Wu, J.; Huang, B.; Zheng, Y. Source tracing and characteristics of industrial VOCs emissions in China. Chin. Environ. Sci. 2012, 32, 48-55.

25. Song, J. Studies on the Adsorption of VOCs by Activated Carbons and the Structure-Function Relationship. Ph.D. Thesis, Central South University, Changsha, China, 2014.

26. Khan, F.I.; Ghoshal, A.K. Removal of Volatile Organic Compounds from polluted air. J. Loss Prevent. Proc. Ind. 2000, 13, 527-545. [CrossRef] 
27. Parmar, G.R.; Rao, N.N. Emerging Control Technologies for Volatile Organic Compounds. Crit. Rev. Environ. Sci. Technol. 2009, 39, 41-78. [CrossRef]

28. Shah, R.; Thonon, B.; Benforado, D. Opportunities for heat exchanger applications in environmental systems. Appl. Therm. Eng. 2000, 20, 631-650. [CrossRef]

29. Dunn, R.F.; El-Halwagi, M.M. Selection of Optimal VOC-condensation Systems. Waste Manag. 1994, 14, 103-113. [CrossRef]

30. Huang, W.; Shi, L.; Hu, Z.; Zheng, Z. Integrated technology of condensation and adsorption for volatile organic compounds recovery. Chem. Eng. 2012, 6, 13-17.

31. Zhang, X.; Gao, B.; Creamer, A.E.; Cao, C.; Li, Y. Adsorption of VOCs onto engineered carbon materials: A review. J. Hazard. Mater. 2017, 338, 102-123. [CrossRef]

32. Wang, S.; Zhang, L.; Long, C.; Li, A. Enhanced adsorption and desorption of vocs vapor on novel micro-mesoporous polymeric adsorbents. J. Coll. Interface Sci. 2014, 428, 185-190. [CrossRef] [PubMed]

33. Serna-Guerrero, R.; Sayari, A. Applications of pore-expanded mesoporoussilica 7, Adsorption of volatile organic compounds. Environ. Sci. Technol. 2007, 41, 4761-4766. [CrossRef] [PubMed]

34. U.S. Environmental Protection Agency Clean Air Technology Center. Choosing an adsorption system for VOC: Carbon, zeolite, or polymers? In Proceedings of the SPIE-The International Society for Optical Engineering, Research Triangle Park, NC, USA, 1 May 1999.

35. Kujawa, J.; Cerneaux, S.; Kujawski, W. Removal of hazardous volatile organic compounds from water by vacuum pervaporation with hydrophobic ceramic membranes. J. Membr. Sci. 2015, 474, 11-19. [CrossRef]

36. Li, Y.X.; Chen, J.Y.; Sun, Y.H. Adsorption of multicomponent volatile organic compounds on semi-coke. Carbon 2008, 46, 858-863.

37. Komori. Preparation of N-acetylmorpholine. J. Chem. Soc. Jpn. Ind. Chem. 1959, 62, 220-225.

38. Chang, F.T.; Lin, Y.C.; Bai, H.; Pei, B.S. Adsorption and desorption characteristics of semiconductor volatile organic compounds on the thermal swing honeycomb zeolite concentrator. J. Air Waste Manage. Assoc. 2003, 53, 1384-1390. [CrossRef]

39. Liu, Y. Study on Ceramic Honeycomb Monolithic Adsorbents for VOCs Adsorption. Master's Thesis, South China University of Technology, Guangzhou, China, 2015.

40. Moretti, E.C. Reduce VOC and HAP emissions. Chem. Eng. Prog. 2002, 98, 30-40.

41. Everaert, K.; Baeyens, J. Catalytic combustion of volatile organic compounds. J. Hazard. Mater. 2004, B109, 113-139. [CrossRef]

42. Liotta, L.F. Catalytic oxidation of volatile organic compounds on supported noble metals. Appl. Catal. B Environ. 2010, 100, 403-412. [CrossRef]

43. Spivey, J.J. Complete Catalytic Oxidation of Volatile Organics. Ind. Eng. Chem. Res. 1987, 26, $2165-2180$. [CrossRef]

44. Zhang, Z.; Jiang, Z.; Shangguan, W. Low-temperature catalysis for VOCs removal in technology andapplication: A state-of-the-art review. Catal. Today 2016, 264, 270-278. [CrossRef]

45. Barbusinski, K.; Kalemba, K.; Kasperczyk, D.; Urbaniec, K.; Kozik, V. Biological methods for odor treatment: A review. J. Clean. Prod. 2017, 152, 223-241. [CrossRef]

46. Mudliar, S.; Giri, B.; Padoley, K.; Satpute, D.; Dixit, R.; Bhatt, P.; Pandey, R.; Juwarkar, A.; Vaidya, A. Bioreactors for treatment of VOCs and odours-A review. J. Environ. Manage. 2010, 91, 1039-1054. [CrossRef]

47. Veerapandian, S.K.P.; Leys, C.; Geyter N., D.; Morent, R. Abatement of VOCs using packed bed non-thermal plasma reactors: A review. Catalysts 2017, 7, 113. [CrossRef]

48. Son, Y.S. Decomposition of VOCs and odorous compounds by radiolysis: A critical review. Chem. Eng. J. 2017, 316, 609-622. [CrossRef]

49. Wang, H. Study on Removal of Miced VOCs in Air by Dielectric Barrier Discharge. Ph.D. Thesis, Dalian University of Technology, Dalian, China, 2009.

50. Hao, J. Control Study of VOCs Regioned Joint Prevention and Control of Atmospheric Pollution. Master's Thesis, Hebei University of Technology, Shijiazhuang, China, 2012.

51. Xi, J.; Wu, J.; Hu, H.; Wang, C. Application status of industrial VOCs gas treatment techniques. China Environ. Sci. 2012, 32, 1955-1960.

52. Hao, Y. Study on The Pharmaceutical and Chemical Industry VOCs and Odor Pollution Characteristics. Master's Thesis, Hebei University of Science and Technology, Shijiazhung, China, 2014. 
53. Department of Environmental Protection of Zhejiang Province. Standards for Emissions of Atmospheric Pollutants from Pharmaceutical Industry in Zhejiang Province; Department of Environmental Protection of Zhejiang Province: Hangzhou, Chain, 2015-2016.

54. Salar-García, M.J.; Ortiz-Martínez, V.M.; Hernández-Fernández, F.J.; de los Ríos, A.P.; Quesada-Medina, J. Ionic liquid technology to recover volatile organic compounds (VOCs). J. Hazard. Mater. 2017, 321, 484-499. [CrossRef]

55. Minella, M.; Baudino, M.; Minero, C. A revised photocatalytic transformation mechanism for chlorinated VOCs: Experimental evidence from $\mathrm{C}_{2} \mathrm{Cl}_{4}$ in the gas phase. Catal. Today 2018, 313, 114-121. [CrossRef]

56. Papaefthimiou, P.; Ioannides, T.; Verykios, X.E. Combustion of non-halogenated volatile organic compounds over group VIII metal catalysts. Appl. Catal. B Environ. 1997, 13, 175-184. [CrossRef]

57. Tabakova, T.; Ilieva, L.; Petrova, P. Complete benzene oxidation over mono and bimetallic Au-Pd catalysts supported on Fe-modified ceria. Chem. Eng. J. 2015, 260, 133-141. [CrossRef]

58. Wang, Y.; Yang, D.; Li, S.; Zhang, L.; Zheng, G.; Guo, L. Layered copper manganese oxide for the efficient catalytic CO and VOCs oxidation. Chem. Eng. J. 2018, 357, 258-268. [CrossRef]

59. Alifanti, M.; Florea, M.; Pârvulescu, V.I. Ceria-based oxides as supports for $\mathrm{LaCoO}_{3}$ perovskite catalysts for total oxidation of VOC. Appl. Catal. B Environ. 2007, 70, 400-405. [CrossRef]

60. Spinicci, R.; Faticanti, M.; Marini, P.; De Rossi, S.; Porta, P. Catalytic activity of $\mathrm{LaMnO}_{3}$ and $\mathrm{LaCoO}_{3}$ perovskites towards VOCs combustion. J. Mol. Catal. A Chem. 2003, 197, 147-155. [CrossRef]

61. Liu, L.; Li, J.; Zhang, H.; Li, L.; Zhou, P.; Meng, X.; Guo, M.; Jia, J.; Sun, T. In situ fabrication of highly active $\gamma-\mathrm{MnO}_{2} / \mathrm{SmMnO}_{3}$ catalyst for deep catalytic oxidation of gaseous benzene, ethylbenzene, toluene, and o-xylene. J. Hazard. Mater. 2019, 362, 178-186. [CrossRef]

62. Shu, Y.; Ji, J.; Xu, Y.; Deng, J.; Huang, H.; He, M.; Leung, D.Y.C.; Wu, M.; Liu, S.; Liu, S.; et al. Promotional role of $\mathrm{Mn}$ doping on catalytic oxidation of VOCs over mesoporous $\mathrm{TiO}_{2}$ under vacuum ultraviolet (VUV) irradiation. Appl. Catal. B Environ. 2018, 220, 78-87. [CrossRef]

63. Ji, J.; Xu, Y.; Huang, H.; He, M.; Liu, S.; Liu, G.; Xie, R.; Feng, Q.; Shu, Y.; Zhan, Y.; et al. Mesoporous $\mathrm{TiO}_{2}$ under VUV irradiation: Enhanced photocatalytic oxidation for VOCs degradation at room temperature. Chem. Eng. J. 2017, 327, 490-499. [CrossRef]

64. Yadav, H.M.; Jung, S.C.; Kim, J.S. Visible light photocatalyticperformance of in situ synthesized graphite- $\mathrm{SiO}_{2}-\mathrm{TiO}_{2}$ composite towards degradation of benzene gas. J. Nanosci. Nanotechnol. 2018, 18, 2032-2036. [CrossRef]

65. Li, J.; Han, S.; Bai, S.; Han, S.; Song, H.; Pu, Y.; Zhu, X.; Chen, W. Effect of Pt/gamma- $\mathrm{Al}_{2} \mathrm{O}_{3}$ catalyst on nonthermal plasma decomposition of benzene and byproducts. Environ. Eng. Sci. 2011, 28, 395-403. [CrossRef]

66. Pei, W.; Liu, Y.; Deng, J.; Zhang, K.; Hou, Z.; Zhao, X.; Dai, H. Partially embedding Pt nanoparticles in the skeleton of $3 \mathrm{DOM} \mathrm{Mn}_{2} \mathrm{O}_{3}$ : An effective strategy for enhancing catalytic stability in toluene combustion. Appl. Catal. B Environ. 2019, 256, 117814-117824. [CrossRef]

67. Hosseini, M.; Barakat, T.; Cousin, R. Catalytic performance of core-shell and alloy Pd-Au nanoparticles for total oxidation of VOC: The effect of metal deposition. Appl. Catal. B Environ. 2012, 111, 218-224. [CrossRef]

68. Lee, D.S.; Chen, Y.W. The mutual promotional effect of $\mathrm{Au}-\mathrm{Pd} / \mathrm{CeO}_{2}$ bimetallic catalysts on destruction of toluene. J. Taiwan Ins. Chem. Eng. 2013, 44, 40-44. [CrossRef]

69. Yang, X.; Yu, X.; Lin, M.; Ma, X.; Ge, M. Enhancement effect of acid treatment on $\mathrm{Mn}_{2} \mathrm{O}_{3}$ catalyst for toluene oxidation. Catal. Today 2019, 327, 254-261. [CrossRef]

70. Huang, H.; Liu, Y.; Tang, W.; Chen, Y. Catalytic activity of nanometer $\mathrm{La}_{1-\mathrm{x}} \mathrm{Sr}_{\mathrm{x}} \mathrm{CoO}_{3}(\mathrm{x}=0,0.2)$ perovskites towards VOCs combustion. Catal. Commun. 2008, 9, 55-59. [CrossRef]

71. Suárez-Vázquez, S.I.; Gil, S.; García-Vargas, J.M.; Cruz-López, A.; Giroir-Fendler, A. Catalytic oxidation of toluene by $\mathrm{SrTi}_{1-\chi} \mathrm{BXO}_{3}(\mathrm{~B}=\mathrm{Cu}$ and $\mathrm{Mn})$ with dendritic morphology synthesized by one pot hydrothermal route. Appl. Catal. B Environ. 2018, 223, 201-208. [CrossRef]

72. Guo, M.; Li, K.; Liu, L.; Zhang, H.; Hu, X.; Min, X.; Jia, J.; Sun, T. Resource utilization of spent ternary lithium-ions batteries: Synthesis of highly active manganese-based perovskite catalyst for toluene oxidation. J. Taiwan Inst. Chem. Eng. 2019, 102, 268-275. [CrossRef]

73. Yang, J.; Li, L.; Yang, X.; Song, S.; Li, J.; Jing, F.; Chu, W. Enhanced catalytic performances of in situ-assembled $\mathrm{LaMnO} / \delta-\mathrm{MnO}_{2}$, hetero-structures for toluene combustion. Catal. Today 2019, 327, 19-27. [CrossRef] 
74. Sihaib, Z.; Puleo, F.; Pantaleo, G.; Parola, V.L.; Valverde, J.L.; Gil, S.; Liotta, L.F.; Giroir-Fendler, A. The effect of citric acid concentration on the properties of $\mathrm{LaMnO}_{3}$ as a catalyst for hydrocarbon oxidation. Catalysts 2019, 9, 226. [CrossRef]

75. Peng, Y.; Zhang, L.; Chen, L.; Yuan, D.; Wang, G.; Meng, X.; Xiao, F.S. Catalytic performance for toluene abatement over Al-rich Beta zeolite supported manganese oxides. Catal. Today 2017, 297, 182-187. [CrossRef]

76. Qin, Y.; Qu, Z.; Dong, C.; Wang, Y.; Huang, N. Highly catalytic activity of Mn/SBA-15 catalysts for toluene combustion improved by adjusting the morphology of supports. J. Environ. Sci. 2019, 76, 208-216. [CrossRef]

77. Lee, Y.E.; Chung, W.C.; Chang, M.B. Photocatalytic oxidation of toluene and isopropanol by LaFeO3/black-TiO 2 . Environ. Sci. Pollut. Res. 2019, 26, 20908-20919. [CrossRef]

78. Bettini, S.; Pagano, R.; Semeraro, P.; Ottolini, M.; Salvatore, L.; Marzo, F.; Lovergine, N.; Giancane, G.; Valli, L. $\mathrm{SiO}_{2}$-Coated $\mathrm{ZnO}$ Nanoflakes Decorated with Ag Nanoparticles for Photocatalytic Water Oxidation. Chem. Eur. J. 2019, 25, 14123-14132. [CrossRef] [PubMed]

79. Li, Z.; Pan, X.; Yi, Z. Photocatalytic oxidation of methane over CuO-decorated ZnO nanocatalysts. J. Mater. Chem. A 2019, 7, 469-475. [CrossRef]

80. Lu, M.; Huang, R.; Wu, J.; Fu, M.; Chen, L.; Ye, D. On the performance and mechanisms of toluene removal by $\mathrm{FeO}_{\mathrm{x}} / \mathrm{SBA}-15$-assisted non-thermal plasma at atmospheric pressure and room temperature. Catal. Today 2015, 242, 274-286. [CrossRef]

81. Nevanperä, T.K.; Ojala, S.; Laitinen, T.; Pitkäaho, S.; Saukko, S.; Keiski, R.L. Catalytic Oxidation of Dimethyl Disulfide over Bimetallic $\mathrm{Cu}-\mathrm{Au}$ and $\mathrm{Pt}-\mathrm{Au}$ Catalysts Supported on $\gamma-\mathrm{Al}_{2} \mathrm{O}_{3}, \mathrm{CeO}_{2}$, and $\mathrm{CeO}_{2}-\mathrm{Al}_{2} \mathrm{O}_{3}$. Catalysts 2019, 9, 603. [CrossRef]

82. Rotter, H.; Landau, M.; Herskowitz, M. Combustion of chlorinated VOC on nanostructured chromia aerogel as catalyst and catalyst support. Environ. Sci. Technol. 2005, 39, 6845-6850. [CrossRef]

83. Dai, Q.; Wang, X.; Lu, G. Low-temperature catalytic destruction of chlorinated VOCs over cerium oxide. Catal. Commun. 2007, 8, 1645-1649. [CrossRef]

84. Ikhlaq, A.; Kasprzyk-Hordern, B. Catalytic ozonation of chlorinated VOCs on ZSM-5 zeolites and alumina: Formation of chlorides. Appl. Catal. B Environ. 2017, 200, 274-282. [CrossRef]

85. Fei, Z.; Cheng, C.; Chen, H.; Li, L.; Yang, Y.; Liu, Q.; Chen, X.; Zhang, Z.; Tang, J.; Cui, M.; et al. Construction of uniform nanodots $\mathrm{CeO}_{2}$ stabilized by porous silica matrix for 1,2-dichloroethane catalytic combustion. Chem. Eng. J. 2019, 370, 916-924. [CrossRef]

86. Stucchi, M.; Galli, F.; Bianchi, C.L.; Pirola, C.; Boffito, D.C.; Biasioli, F.; Capucci, V. Simultaneous photodegradation of VOC mixture by $\mathrm{TiO}_{2}$ powders. Chemosphere 2018, 193, 198-206. [CrossRef] [PubMed]

87. Zhu, X.; Tu, X.; Mei, D.; Zheng, C.; Zhou, J.; Gao, X.; Luo, Z.; Ni, M.; Cen, K. Investigation of hybrid plasma-catalytic removal of acetone over $\mathrm{CuO} /-\mathrm{Al}_{2} \mathrm{O}_{3}$ catalysts using response surface method. Chemosphere 2016, 155, 9-17. [CrossRef] [PubMed]

88. Li, Z.; Meng, X.; Zhang, Z. Fabrication of surface hydroxyl modified g- $\mathrm{C}_{3} \mathrm{~N}_{4}$ with enhanced photocatalytic oxidation activity. Catal. Sci. Technol. 2019, 9, 3979-3993. [CrossRef]

89. Wu, Y.; Shi, S.; Yuan, S.; Yuan, S.; Bai, T.; Xing, S. Insight into the enhanced activity of Ag/ $\mathrm{NiO}_{\mathrm{x}}-\mathrm{MnO}_{2}$ for catalytic oxidation of o-xylene at low temperatures. Appl. Surf. Sci. 2019, 479, 1262-1269. [CrossRef]

90. Yang, S.; Zhao, H.; Dong, F.; Zha, F.; Tang, Z. Highly efficient catalytic combustion of o-dichlorobenzene over three-dimensional ordered mesoporous cerium manganese bimetallic oxides: A new concept of chlorine removal mechanism. Mol. Catal. 2019, 463, 119-129. [CrossRef]

91. He, D.; Zhao, Y.; Yang, S.; Mei, Y.; Yu, J.; Liu, J.; Chen, D.; He, S.; Luo, Y. Enhancement of catalytic performance and resistance to carbonaceous deposit of lanthanum (La) doped HZSM-5 catalysts for decomposition of methyl mercaptan. Chem. Eng. J. 2018, 336, 579-586. [CrossRef]

92. Liu, N.; Shi, D.; Zhang, R.; Li, Y.; Chen, B. Highly selective catalytic combustion of acrylonitrile towards nitrogen over Cu-modified zeolites. Catal. Today 2018, 332, 201-213. [CrossRef]

93. Fiorenza, R.; Crisafulli, C.; Condorelli, G.G.; Lupo, F.; Scire, S. Au-Ag/CeO 2 and $\mathrm{Au}-\mathrm{Cu} / \mathrm{CeO} 2$ Catalysts for Volatile Organic Compounds Oxidation and CO Preferential Oxidation. Catal. Lett. 2015, 145, 1691-1702. [CrossRef]

94. Yao, J.; Lu, H.; Xiao, Y.; Hou, B.; Li, D.; Jia, L. Sub-molten salt-acid treatment of $\mathrm{LaCoO}_{3}$ for a highly active catalyst towards propane combustion. Catal. Commun. 2019, 128, 10578-10583. [CrossRef]

95. Zhang, K.; Peng, X.; Yang, H.; Wang, X.; Zhang, Y.; Zheng, Y.; Xiao, Y.; Jiang, L. Effect of $\mathrm{MnO}_{2}$ morphology on its catalytic performance in lean methane combustion. Mater. Res. Bull. 2018, 111, 338-341. [CrossRef] 
96. Wei, Y.; Ni, L.; Li, M.; Zhao, J. A template-free method for preparation of $\mathrm{MnO}_{2}$ catalysts with high surface areas. Catal. Today 2017, 297, 188-192. [CrossRef]

97. Sinquin, G.; Petit, C.; Hindermann, J.P.; Kiennemann, A. Study of the formation of $\operatorname{LaMO}_{3}(\mathrm{M}=\mathrm{Co}$, $\mathrm{Mn})$ perovskites by propionates precursors: Application to the catalytic destruction of chlorinated VOCs. Catal. Today 2001, 70, 183-196. [CrossRef]

98. Alberici, R.M.; Jardim, W.F. Photocatalytic destruction of VOCs in the gas-phase using titanium dioxide. Appl. Catal. B Environ. 1997, 14, 55-68. [CrossRef]

99. Fujimoto, T.M.; Ponczek, M.; Rochetto, U.L.; Landers, R.; Tomaz, E. Photocatalytic oxidation of selected gas-phase VOCs using UV light, $\mathrm{TiO}_{2}$, and $\mathrm{TiO}_{2} / \mathrm{Pd}$. Environ. Sci. Pollut. Res. 2017, 24, 6390-6396. [CrossRef]

100. Qian, X.; Ren, M.; Yue, D.; Zhu, Y.; Han, Y.; Bian, Z.; Zhao, Y. Mesoporous $\mathrm{TiO}_{2}$ films coated on carbon foam based on waste polyurethane for enhanced photocatalytic oxidation of VOCs. Appl. Catal. B Environ. 2017, 212, 1-6. [CrossRef]

101. Zhang, L.; Peng, Y.; Zhang, J.; Chen, L.; Meng, X.; Xiao, F.S. Adsorptive and catalytic properties in the removal of volatile organic compounds over zeolite-based materials. Chin. J. Catal. 2016, 37, 800-809. [CrossRef]

102. Huang, H.; Huang, H.; Zhan, Y.; Liu, G.; Wang, X.; Lu, H.; Xiao, L.; Feng, Q.; Leung, D.Y.C. Efficient degradation of gaseous benzene by VUV photolysis combined with ozone-assisted catalytic oxidation: Performance and mechanism. Appl. Catal. B Environ. 2016, 186, 62-68. [CrossRef]

103. Song, H.; Peng, Y.; Liu, S.; Bai, S.; Hong, X.; Li, J. The Roles of Various Plasma Active Species in Toluene Degradation by Non-thermal Plasma and Plasma Catalysis. Plasma Chem. Plasma Process. 2019, 39, 1469-1482. [CrossRef]

104. Wang, L.; Wang, C.; Xie, H.; Zhan, W.; Guo, Y. Catalytic combustion of vinyl chloride over Sr doped $\mathrm{LaMnO}_{3}$. Catal. Today 2018, 327, 190-195. [CrossRef]

105. Kołodziej, A.; Łojewska, J. Optimization of structured catalyst carriers for VOC combustion. Catal. Today 2005, 105, 378-384. [CrossRef]

106. Joung, H.J.; Kim, J.H.; Oh, J.S.; You, D.W.; Park, H.O.; Jung, K.W. Catalytic oxidation of VOCs over CNT-supported platinum nanoparticles. Appl. Surf. Sci. 2014, 290, 267-273. [CrossRef]

107. Kim, S.C.; Shim, W.G. Properties and performance of Pd based catalysts for catalytic oxidation of volatile organic compounds. Appl. Catal. B Environ. 2009, 92, 429-436. [CrossRef]

108. Bedi, J.; Rosas, J.M.; Rodríguez-Mirasol, J.; Cordero, T. Pd supported on mesoporous activated carbons with high oxidation resistance as catalysts for toluene oxidation. Appl. Catal. B Environ. 2010, 94, 8-18. [CrossRef]

109. Usón, L.; Colmenares, M.G.; Hueso, J.L.; Sebastián, V.; Balas, F.; Arruebo, M.; Santamaría, J. VOCs abatement using thick eggshell Pt/SBA-15 pellets with hierarchical porosity. Catal. Today 2014, 227, 179-186. [CrossRef]

110. Carrillo, A.M.; Carriazo, J.G. Cu and Co oxides supported on halloysite for the total oxidation of toluene. Appl. Catal. B Environ. 2015, 164, 443-452. [CrossRef]

111. Kucherov, A.V.; Sinev, I.M.; Ojala, S.; Keiski, R.L.; Kustov, M. Adsorptive-catalytic removal of $\mathrm{CH}_{3} \mathrm{OH}$, $\mathrm{CH}_{3} \mathrm{SH}$, and $\mathrm{CH}_{3} \mathrm{SSCH}_{3}$ from air over the bifunctional system noble metals/HZSM-5. Stud. Surf. Sci. Catal. 2007, 170, 1129-1136.

112. Wang, J. Study on Supported Ruthenium Catalysts for the Catalytic Oxidation of VOCs. Ph.D. Thesis, Institute of Process Engineering, CAS, China, 2016.

113. Abdelouahab-Reddam, Z.; Mail, R.E.; Coloma, F.; Sepúlveda-Escribano, A. Platinum supported on highly-dispersed ceria on activated carbon for the total oxidation of VOCs. Appl. Catal. A Gen. 2015, 494, 87-94. [CrossRef]

114. Piumetti, M.; Fino, D.; Russo, N. Mesoporous manganese oxides prepared by solution combustion synthesis as catalysts for the total oxidation of VOCs. Appl. Catal. B Environ. 2015, 163, 277-287. [CrossRef]

115. Grbic, B.; Radic, N.; Terlecki-Baricevic, A. Kinetics of deep oxidation of n-hexane and toluene over $\mathrm{Pt} / \mathrm{Al}_{2} \mathrm{O}_{3}$ catalysts: Oxidation of mixture. Appl. Catal. B Environ. 2004, 50, 161-166. [CrossRef]

116. Patterson, M.J.; Angove, D.E.; Cant, N.W. The effect of carbon monoxide on the oxidation of four C6 to C8 hydrocarbons over platinum, palladium and rhodium. Appl. Catal. B Environ. 2000, 26, 47-57. [CrossRef]

117. McCabe, R.W.; Mitchell, P.J. Exhaust-catalyst development for methanol-fueled vehicles: 1 . A comparative study of methanol oxidation over alumina-supported catalysts containing group 9, 10, and 11 metals. Appl. Catal. 1986, 27, 83-98. [CrossRef] 
118. Liotta, L.F.; Ousmane, M.; Di Carlo, G.; Pantaleo, G.; Deganello, G.; Marcì, G.; Retailleau, L.; Giroir-Fendler, A. Total oxidation of propene at low temperature over $\mathrm{Co}_{3} \mathrm{O}_{4}-\mathrm{CeO}_{2}$ mixed oxides: Role of surface oxygen vacancies and bulk oxygen mobility in the catalytic activity. Appl. Catal. A Gen. 2008, 347, 81-88. [CrossRef]

119. Liotta, L.F.; Ousmane, M.; Di Carlo, G.; Pantaleo, G.; Deganello, G.; Boreave, A.; Giroir-Fendler, A. Catalytic removal of toluene over $\mathrm{Co}_{3} \mathrm{O}_{4}-\mathrm{CeO}_{2}$ mixed oxide catalysts: Comparison with $\mathrm{Pt} / \mathrm{Al}_{2} \mathrm{O}_{3}$. Catal. Lett. 2009, 127, 270-276. [CrossRef]

120. Mitsui, T.; Tsutsui, K.; Matsui, T.; Kikuchi, R.; Eguchi, K. Catalytic abatement of acetaldehyde over oxide-supported precious metal catalysts. Appl. Catal. B Environ. 2008, 78, 158-165. [CrossRef]

121. Zhang, C.; He, H. A comparative study of $\mathrm{TiO}_{2}$ supported noble metal catalysts for the oxidation of formaldehyde at room temperature. Catal. Today 2007, 126, 345-350. [CrossRef]

122. Zhang, C.B.; He, H.; Tanaka, K. Catalytic performance and mechanism of a Pt/TiO 2 catalyst for the oxidation of formaldehyde at room temperature. Appl. Catal. B Environ. 2006, 65, 37-43. [CrossRef]

123. Agarwal, S.K.; Spivey, J.J.; Butt, J.B. Catalyst deactivation during deep oxidation of chlorohydrocarbons. Appl. Catal. A Gen. 1992, 82, 259-275. [CrossRef]

124. Petrosius, S.C.; Drago, R.S.; Young, V.; Grunewald, G.C. Low-temperature decomposition of some halogenated hydrocarbons using metal oxide/porous carbon catalysts. J. Am. Chem. Soc. 1993, 115, 6131-6137. [CrossRef]

125. Sedjame, H.J.; Fontaine, C.; Lafaye, G.; Barbier, J.J. On the promoting effect of the addition of ceria to platinum based alumina catalysts for VOCs oxidation. Appl. Catal. B Environ. 2014, 144, 233-242. [CrossRef]

126. Carabineiro, S.; Chen, X.; Konsolakis, M.; Psarras, A.; Tavares, P.; Orf ao, J.; Pereira, M.; Figueiredo, J. Catalytic oxidation of toluene on Ce-Co and La-Co mixed oxides synthesized by exotemplating and evaporation methods. Catal. Today 2015, 244, 161-171. [CrossRef]

127. Carabineiro, S.; Chen, X.; Martynyuk, O.; Bogdanchikova, N.; Avalos-Borja, M.; Pestryakov, A.; Tavares, P.; Orf ao, J.; Pereira, M.; Figueiredo, J. Gold supported on metal oxides for volatile organic compounds total oxidation. Catal. Today 2015, 244, 103-114. [CrossRef]

128. Castano, M.H.; Molina, R.; Moreno, S. Catalytic oxidation of VOCs on $\mathrm{MnMgAlO}_{\mathrm{x}}$ mixed oxides obtained by auto-combustion. J. Mol. Catal. A Chem. 2015, 398, 358-367. [CrossRef]

129. Chen, H.; Zhang, H.; Yan, Y. Fabrication of porous copper/manganese binary oxides modified ZSM-5 membrane catalyst and potential application in the removal of VOCs. Chem. Eng. J. 2014, 254, 133-142. [CrossRef]

130. Solsona, B.; Davies, T.E.; Garcia, T.; Vázquez, I.; Dejoz, A.; Taylor, S.H. Total oxidation of propane using nanocrystalline cobalt oxide and supported cobalt oxide catalysts. Appl. Catal. B Environ. 2008, 84, 176-184. [CrossRef]

131. Liu, Q.; Wang, L.C.; Chen, M.; Cao, Y.; He, H.Y.; Fan, K.N. Dry citrate precursor synthesized nanocrystalline cobalt oxide as highly active catalyst for total oxidation of propane. J. Catal. 2009, 263, 104-113. [CrossRef]

132. Tseng, T.K.; Wang, L.; Ho, C.T.; Chu, H. The destruction of dichloroethane over a g-alumina supported manganese oxide catalyst. J. Hazard. Mater. 2010, 178, 1035-1040. [CrossRef]

133. Krishnamoorthy, S.; Rivas, J.A.; Amiridis, M.D. Catalytic oxidation of 1, 2-dichlorobenzene over supported transition metal oxides. J. Catal. 2000, 193, 264-272. [CrossRef]

134. Lahousse, C.; Bernier, A.; Grange, P.; Delmon, B.; Papaefthimiou, P.; Ioannides, T.; Verykios, X. Evaluation of g- $\mathrm{MnO}_{2}$ as a VOC removal catalyst: Comparison with a noble metal catalyst. J. Catal. 1998, 178, $214-225$. [CrossRef]

135. Parida, K.; Samal, A. Catalytic combustion of volatile organic compounds on Indian Ocean manganese nodules. Appl. Catal. A Gen. 1999, 182, 249-256. [CrossRef]

136. Luo, J.; Zhang, Q.; Huang, A.; Suib, S.L. Total oxidation of volatile organic compounds with hydrophobic cryptomelane-type octahedral molecular sieves. Microporous Mesoporous Mater. 2000, 35-36, 209-217. [CrossRef]

137. Aguero, F.N.; Scian, A.; Barbero, B.P.; Cadús, L.E. Influence of the support treatment on the behavior of $\mathrm{MnO}_{x} / \mathrm{Al}_{2} \mathrm{O}_{3}$ catalysts used in VOC combustion. Catal. Lett. 2009, 128, 268-280. [CrossRef]

138. Kang, M.; Park, E.D.; Kim, J.M.; Yie, J.E. Manganese oxide catalysts for NOx reduction with NH3 at low temperatures. Appl. Catal. A Gen. 2007, 327, 261-269. [CrossRef]

139. Miranda, B.; Díaz, E.; Ordonez, S.; Vega, A.; Díez, F.V. Oxidation of trichloroethene over metal oxide catalysts: Kinetic studies and correlation with adsorption properties. Chemosphere 2007, 66, 1706-1715. [CrossRef] 
140. Sun, H.; Liu, Z.; Chen, S.; Quan, X. The role of lattice oxygen on the activity and selectivity of the OMS-2 catalyst for the total oxidation of toluene. Chem. Eng. J. 2015, 270, 58-65. [CrossRef]

141. Cordi, E.M.; O'Neill, P.J.; Falconer, J.L. Transient oxidation of volatile organic compounds on a $\mathrm{CuO} / \mathrm{Al}_{2} \mathrm{O}_{3}$ catalyst. Appl. Catal. B Environ. 1997, 14, 23-36. [CrossRef]

142. Hutchings, G.J.; Taylor, S.H. Designing oxidation catalysts. Catal. Today 1999, 49, 105-113. [CrossRef]

143. Heynderickx, P.M.; Thybaut, J.W.; Poelman, H.; Poelman, D.; Marin, G.B. The total oxidation of propane over supported $\mathrm{Cu}$ and Ce oxides: A comparison of single and binary metal oxides. J. Catal. 2010, 272, 109-120. [CrossRef]

144. Sinha, A.K.; Suzuki, K. Novel mesoporous chromium oxide for VOCs elimination. Appl. Catal. B Environ. 2007, 70, 417-422. [CrossRef]

145. Padilla, A.M.; Corella, J.; Toledo, J.M. Total oxidation of some chlorinated hydrocarbons with commercial chromia based catalysts. Appl. Catal. B Environ. 1999, 22, 107-121. [CrossRef]

146. Yim, S.D.; Chang, K.-H.; Nam, I.S. Deactivation of chromium oxide catalyst for the removal of perchloroethylene (PCE). Stud. Surf. Sci. Catal. 2001, 139, 173-180.

147. Gorte, R.J. Ceria in catalysis: From automotive applications to the water-gas shift reaction. AlChE J. 2010, 56, 1126-1135. [CrossRef]

148. Zimmer, P.; Tschope, A.; Birringer, R. Temperature-programmed reaction spectroscopy of ceria-and $\mathrm{Cu} /$ ceria-supported oxide catalyst. J. Catal. 2002, 205, 339-345. [CrossRef]

149. Huang, H.; Xu, Y.; Feng, Q.; Leung, D.Y.C. Low temperature catalytic oxidation of volatile organic compounds: A review. Catal. Sci. Technol. 2015, 5, 2649-2669. [CrossRef]

150. Li, H.; Lu, G.; Dai, Q.; Wang, Y.; Guo, Y.; Guo, Y. Hierarchical organization and catalytic activity of high-surface-area mesoporous ceria microspheres prepared via hydrothermal routes. ACS Appl. Mater. Interfaces 2010, 2, 838-846. [CrossRef]

151. Jones, J.; Ross, J.R. The development of supported vanadia catalysts for the combined catalytic removal of the oxides of nitrogen and of chlorinated hydrocarbons from flue gases. Catal. Today 1997, 35, 97-105. [CrossRef]

152. Delaigle, R.; Debecker, D.P.; Bertinchamps, F.; Gaigneaux, E.M. Revisiting the behaviour of vanadia-based catalysts in the abatement of (chloro)-aromatic pollutants: Towards an integrated understanding. Top. Catal. 2009, 52, 501-516. [CrossRef]

153. Solsona, B.; Garcia, T.; Aylon, E.; Dejoz, A.M.; Vazquez, I.; Agouram, S.; Davies, T.E.; Taylor, S.H. Promoting the activity and selectivity of high surface area Ni-Ce-O mixed oxides by gold deposition for VOC catalytic combustion. Chem. Eng. J. 2011, 175, 271-278. [CrossRef]

154. Delimaris, D.; Ioannides, T. VOC oxidation over $\mathrm{MnO}_{\mathrm{x}}-\mathrm{CeO}_{2}$ catalysts prepared by a combustion method. Appl. Catal. B Environ. 2008, 84, 303-312. [CrossRef]

155. Morales, M.R.; Barbero, B.P.; Cadús, L.E. Total oxidation of ethanol and propane over Mn-Cu mixed oxide catalysts. Appl. Catal. B Environ. 2006, 67, 229-236. [CrossRef]

156. Vasile, A.; Bratan, V.; Hornoiu, C.; Caldararu, M.; Ionescu, N.I.; Yuzhakova, T.; Redey, A. Electrical and catalytic properties of cerium etin mixed oxides in CO depollution reaction. Appl. Catal. B Environ. 2013, 140, 25-31. [CrossRef]

157. Tang, W.; Wu, X.; Li, S.; Li, W.; Chen, Y. Porous Mn-Co mixed oxide nanorod as a novel catalyst with enhanced catalytic activity for removal of VOCs. Catal. Commun. 2014, 56, 134-138. [CrossRef]

158. Larsson, P.O.; Andersson, A. Complete oxidation of CO, ethanol, and ethyl acetate over copper oxide supported on titania and ceria modified titania. J. Catal. 1998, 179, 72-89. [CrossRef]

159. Hu, C.; Zhu, Q.; Jiang, Z.; Zhang, Y.; Wang, Y. Preparation and formation mechanism of mesoporous $\mathrm{CuOeCeO} 2$ mixed oxides with excellent catalytic performance for removal of VOCs. Microporous Mesoporous Mater. 2008, 113, 427-434. [CrossRef]

160. Delimaris, D.; Ioannides, T. VOC oxidation over $\mathrm{CuO}-\mathrm{CeO}_{2}$ catalysts prepared by a combustion method. Appl. Catal. B Environ. 2009, 89, 295-302. [CrossRef]

161. Rao, T.; Shen, M.; Jia, L.; Hao, J.; Wang, J. Oxidation of ethanol over Mn-Ce-O and Mn-Ce-Zr-O complex compounds synthesized by solegel method. Catal. Commun. 2007, 8, 1743-1747. [CrossRef]

162. Tang, X.; Li, Y.; Huang, X.; Xu, Y.; Zhu, H.; Wang, J.; Shen, W. $\mathrm{MnO}_{\mathbf{x}}-\mathrm{CeO}_{2}$ mixed oxide catalysts for complete oxidation of formaldehyde: Effect of preparation method and calcination temperature. Appl. Catal. B Environ. 2006, 62, 265-273. [CrossRef] 
163. Picasso, G.; Gutierrez, M.; Pina, M.; Herguido, J. Preparation and characterization of Ce-Zr and Ce-Mn based oxides for n-hexane combustion: Application to catalytic membrane reactors. Chem. Eng. J. 2007, 126, 119-130. [CrossRef]

164. Chen, H.; Sayari, A.; Adnot, A.; Larachi, F. Composition activity effects of Mn-Ce-O composites on phenol catalytic wet oxidation. Appl. Catal. B Environ. 2001, 32, 195-204. [CrossRef]

165. Yang, P.; Yang, S.; Shi, Z.; Meng, Z.; Zhou, R. Deep oxidation of chlorinated VOCs over $\mathrm{CeO}_{2}$-based transition metal mixed oxide catalysts. Appl. Catal. B Environ. 2015, 162, 227-235. [CrossRef]

166. Li, J.; Zhao, P.; Liu, S. $\mathrm{SnO}_{\mathrm{x}}-\mathrm{MnO}_{\mathrm{x}}-\mathrm{TiO}_{2}$ catalysts with high resistance to chlorine poisoning for low-temperature chlorobenzene oxidation. Appl. Catal. A Gen. 2014, 482, 363-369. [CrossRef]

167. Rao, G.R.; Sahu, H.R.; Mishra, B.G. Surface and catalytic properties of Cu-Ce-O composite oxides prepared by combustion method. Coll. Surf. A Physicochem. Eng. Asp. 2003, 220, 261-269. [CrossRef]

168. Jiang, X.; Lu, G.; Zhou, R.; Mao, J.; Chen, Y.; Zheng, X. Studies of pore structure, temperature-programmed reduction performance, and micro-structure of $\mathrm{CuO} / \mathrm{CeO}_{2}$ catalysts. Appl. Surf. Sci. 2001, 173, 208-220.

169. Zheng, X.C.; Wu, S.H.; Wang, S.P.; Wang, S.R.; Zhang, S.M.; Huang, W.P. The preparation and catalytic behavior of copperecerium oxide catalysts for low-temperature carbon monoxide oxidation. Appl. Catal. A Gen. 2005, 283, 217-223. [CrossRef]

170. Pecchi, G.; Reyes, P.; Zamora, R.; Cadus, L.E.; Fierro, J.L.G. Surface properties and performance for VOCs combustion of $\mathrm{LaFe}_{1-\mathrm{y}} \mathrm{Ni}_{\mathrm{y}} \mathrm{O}_{3}$ perovskite oxides. J. Solid State Chem. 2008, 181, 905-912. [CrossRef]

171. Wei, T. Study on Catalytic Combustion of VOCs over Perovskite Catalysts. Master's Thesis, Zhejiang University of Technology, Zhejiang, China, 2005.

172. Beauchet, R.; Magnoux, P.; Mijoin, J. Catalytic oxidation of volatile organic compounds (VOCs) mixture (isopropanol/o-xylene) on zeolite catalysts. Catal. Today 2007, 124, 118-123. [CrossRef]

173. Rachapudi, R.; Chintawar, P.S.; Greene, H.L. Aging and Structure/Activity Characteristics of CR-ZSM-5 Catalysts during Exposure to Chlorinated VOCs. J. Catal. 1999, 185, 58-72. [CrossRef]

174. Muniandy, L.; Adam, F.; Mohamed, A.R.; Iqbal, A.; Rahman, N.R.A. $\mathrm{Cu}^{2+}$ coordinated graphitic carbon nitride $\left(\mathrm{Cu}-\mathrm{g}-\mathrm{C}_{3} \mathrm{~N}_{4}\right)$ nanosheets from melamine for the liquid phase hydroxylation of benzene and VOCs. Appl. Surf. Sci. 2017, 398, 43-55. [CrossRef]

175. Blanch-Raga, N.; Palomares, A.E.; Martínez-Triguero, J.; Valencia, S. Cu and Co modified beta zeolite catalysts for the trichloroethylene oxidation. Appl. Catal. B Environ. 2016, 187, 90-97. [CrossRef]

176. Lopez-Fonseca, R.; Aranzabal, A.; Steltenpohl, P.; Gutierrez-Ortiz, J.I.; Gonzalez Velasco, J.R. Performance of zeolites and product selectivity in the gas-phase oxidation of 1,2-dichloroethane. Catal. Today 2000, 62, 367-377. [CrossRef]

177. Kullavanijayam, E.; Trimm, D.L.; Cant, N.W. Adsocat: Adsorption/catalytic combustion for VOC and odour control. Stud. Surf. Sci. Catal. 2000, 130, 569-574.

178. Fujishima, A.; Honda, K. Electrochemical photolysis of water at a semiconductorelectrode. Nature 1972, 238, 37-38. [CrossRef] [PubMed]

179. Wang, D.; Hou, P.; Yang, P.; Cheng, X. BiOBr@SiO 2 flower-like nanospheres chemically-bonded on cement-based materials for photocatalysis. Appl. Surf. Sci. 2018, 430, 539-548. [CrossRef]

180. Li, Y.; Wu, X.; Li, J.; Wang, K.; Zhang, G. Z-scheme g-C3N4@CsxWO 3 heterostructure as smart window coating for UV isolating, Vis penetrating, NIR shielding and full spectrum photocatalytic decomposing VOCs. Appl. Catal. B Environ. 2018, 229, 218-226. [CrossRef]

181. Mishra, A.; Mehta, A.; Kainth, S.; Basu, S. Effect of different plasmonic metals on photocatalytic degradation of volatile organic compounds (VOCs) by bentonite/ $\mathrm{M}-\mathrm{TiO}_{2}$ nanocomposites, under UV/visible light. Appl. Clay Sci. 2018, 153, 144-153. [CrossRef]

182. Song, S.; Lu, C.; Wu, X.; Jiang, S.; Sun, C.; Le, Z. Strong base g- $\mathrm{C}_{3} \mathrm{~N}_{4}$ with perfect structure for photocatalytically eliminating formaldehyde under visible-light irradiation. Appl. Catal. B Environ. 2018, 227, 145-152. [CrossRef]

183. Bhatkhande, D.S.; Pangarkar, V.G.; Beenackers, A.A.C.M. Photocatalytic degradation for environmental applications-A review. J. Chem. Technol. Biotechnol. Int. Res. Process Environ. Clean Technol. 2001, 77, 107-116. [CrossRef]

184. Choi, H.; Stathatos, E.; Dionysiou, D.D. Sol-gel preparation of mesoporous photocatalytic $\mathrm{TiO}_{2}$ films and $\mathrm{TiO}_{2} / \mathrm{Al}_{2} \mathrm{O}_{3}$ composite membranes for environmental applications. Appl. Catal. B Environ. 2006, 63, 60-67. [CrossRef] 
185. Fujishima, A. $\mathrm{TiO}_{2}$ photocatalysis and related surface phenomena. Surf. Sci. Rep. 2008, 63, 515-582. [CrossRef]

186. Minero, C. Surface modified photocatalysts, in environmental photochemistry part III. In The Handbook of Environmental Chemistry; Bahnemann, D.W., Robertson, K.J., Eds.; Springer: Berlin/Heidelberg, Germany, 2015; Volume 35, pp. 23-44.

187. Tejasvi, R.; Sharma, M.; Upadhyay, K. Passive photo-catalytic destruction of air-borne VOCs in high traffic areas using $\mathrm{TiO}_{2}$-coated flexible PVC sheet. Chem. Eng. J. 2015, 262, 875-881. [CrossRef]

188. Li, F.B.; Li, X.Z.; Ao, C.H.; Lee, S.C.; Hou, M.F. Enhanced photocatalytic degradation of VOCs using $\mathrm{Ln}^{3+}-\mathrm{TiO}_{2}$ catalysts for indoor air purification. Chemosphere 2005, 59, 787-800. [CrossRef]

189. Héqueta, V.; Raillarda, C.; Debonoa, O.; Thévenet, F.; Locoge, N.; Le Coq, L. Photocatalytic oxidation of VOCs at ppb level using a closed-loop reactor: The mixture effect. Appl. Catal. B Environ. 2018, 226, 473-486. [CrossRef]

190. Huang, L.; Nakajo, K.; Ozawa, S.; Matsuda, H. Decomposition of dichloromethane in a wire-in-tube pulsed corona reactor. Environ. Sci. Technol. 2001, 35, 1276-1281. [CrossRef]

191. Norberg, A. Modeling current pulse shape and energy in surface discharges. IEEE Trans. Ind. Appl. 1992, 28, 498-503. [CrossRef]

192. Rousseau, A.; Dantier, A.; Gatilova, L.; Ionikh, Y.; Röpcke, J.; Tolmachev, Y. On NOx production and volatile organic compound removal in a pulsed microwave discharge in air. Plasma Sour. Sci. Technol. 2005, 14, 70-75. [CrossRef]

193. Kogelschatz, U. Dielectric-barrier discharges: Their history, discharge physics, and industrial applications. Plasma Chem. Plasma Process. 2003, 23, 1-46. [CrossRef]

194. Yamamoto, T.; Ramanathan, K.; Lawless, P.A.; Ensor, D.S.; Newsome, J.R.; Plaks, N.; Ramsey, G.H. Control of volatile organic compounds by an ac energized ferroelectric pellet reactor and a pulsed corona reactor. IEEE Trans. Ind. Appl. 1992, 28, 528-534. [CrossRef]

195. Ding, H.-X.; Zhu, A.-M.; Yang, X.-F.; Li, C.-H.; Xu, Y. Removal of formaldehyde from gas streams via packed-bed dielectric barrier discharge plasmas. J. Phys. D App. Phys. 2005, 38, 4160-4167. [CrossRef]

196. Kim, H.H.; Ogata, A.; Futamura, S. Oxygen partial pressure-dependent behavior of various catalysts for the total oxidation of VOCs using cycled system of adsorption and oxygen plasma. Appl. Catal. B Environ. 2008, 79, 356-367. [CrossRef]

197. Futamura, S.; Yamamoto, T.; Lawless, P.A. Towards understanding of VOC decomposition mechanisms using nonthermal plasmas. In Proceedings of the 1995 Thirtieth IAS Annual Meeting IEEE Conference Record of Industry Applications Conference, Orlando, FL, USA, 8-12 October 1995; Volume 2, pp. 1453-1458.

198. Zheng, C.; Zhu, X.; Gao, X.; Liu, L.; Chang, Q.; Luo, Z.; Cen, K. Experimental study of acetone removal by packed-bed dielectric barrier discharge reactor. J. Ind. Eng. Chem. 2014, 20, 2761-2768. [CrossRef]

199. Lee, B.Y.; Park, S.H.; Lee, S.C.; Kang, M.; Choung, S.J. Decomposition of benzene by using a discharge plasma-photocatalyst hybrid system. Catal. Today 2004, 93-95, 769-776. [CrossRef]

200. Gandhi, M.S.; Ananth, A.; Mok, Y.S.; Song, J.I.; Park, K.H. Effect of porosity of $\alpha$-alumina on non-thermal plasma decomposition of ethylene in a dielectric-packed bed reactor. Res. Chem. Intermed. 2014, 40, 1483-1493. [CrossRef]

201. Hu, J.; Jiang, N.; Li, J.; Shang, K.; Lu, N.; Wu, Y. Degradation of benzene by bipolar pulsed series surface/packed-bed discharge reactor over $\mathrm{MnO}_{2}-\mathrm{TiO}_{2}$ /zeolite catalyst. Chem. Eng. J. 2016, 293, 216-224. [CrossRef]

202. Ogata, A.; Yamanouchi, K.; Mizuno, K.; Kushiyama, S.; Yamamoto, T. Oxidation of dilute benzene in an alumina hybrid plasma reactor at atmospheric pressure. Plasma Chem. Plasma Process. 1999, 19, 383-394. [CrossRef]

203. Zhu, X.; Gao, X.; Qin, R.; Zeng, Y.; Qu, R.; Zheng, C.; Tu, X. Plasma-catalytic removal of formaldehyde over $\mathrm{Cu}-\mathrm{Ce}$ catalysts in a dielectric barrier discharge reactor. Appl. Catal. B Environ. 2015, 170-171, 293-300. [CrossRef]

204. Bradford, M.C.J.; Vannice, M.A. Estimation of CO heats of adsorption on metal surfaces from vibrational spectra. Ind. Eng. Chem. Res. 1996, 35, 3171-3178. [CrossRef]

205. An, H.T.Q.; Huu, T.P.; Le Van, T.; Cormier, J.M.; Khacef, A. Application of atmospheric non thermal plasma-catalysis hybrid system for air pollution control: Toluene removal. Catal. Today 2011, 176, 474-477.

206. Futamura, S.; Einaga, H.; Kabashima, H.; Hwan, L.Y. Synergistic effect of silent discharge plasma and catalysts on benzene decomposition. Catal. Today 2004, 89, 89-95. [CrossRef] 
207. Wu, J.; Huang, Y.; Xia, Q.; Li, Z. Decomposition of toluene in a plasma catalysis system with $\mathrm{NiO}_{1} \mathrm{MnO}_{2}$, $\mathrm{CeO}_{2}, \mathrm{Fe}_{2} \mathrm{O}_{3}$, and $\mathrm{CuO}$ catalysts. Plasma Chem. Plasma Process. 2013, 33, 1073-1082. [CrossRef]

208. Zhao, D.-Z.; Li, X.-S.; Shi, C.; Fan, H.-Y.; Zhu, A.-M. Low-concentration formaldehyde removal from air using a cycled storage-discharge (CSD) plasma catalytic process. Chem. Eng. Sci. 2011, 66, 3922-3929. [CrossRef]

209. Ran, L.; Wang, Z.; Wang, X. The effect of Ce on catalytic decomposition of chlorinated methane over $\mathrm{RuO}_{\mathrm{x}}$ catalysts. Appl. Catal. A gen. 2014, 470, 442-450. [CrossRef]

210. Asilevi, P.J.; Yi, C.W.; Li, J.; Nawaz, M.I.; Wang, H.J.; Yin, L.; Junli, Z. Decomposition of formaldehyde in strong ionization non-thermal plasma at atmospheric pressure. Int. J. Environ. Sci. Technol. 2020, 17, 765-776. [CrossRef]

211. Zhu, T.; Li, J.; Jin, Y.; Liang, Y.; Ma, G. Decomposition of benzene by non-thermal plasma processing: Photocatalyst and ozone effect. Int. J. Environ. Sci. Technol. 2008, 5, 375-384. [CrossRef]

212. Urashima, K.; Kostov, K.G.; Chang, J.S.; Okayasu, Y.; Iwaizumi, T.; Yoshimura, K.; Kato, T. Removal of $\mathrm{C}_{2} \mathrm{~F}_{6}$ from a semiconductor process flue gas by a ferroelectric packed-bed barrier discharge reactor with an adsorber. IEEE Trans. Ind. Appl. 2001, 37, 1456-1463. [CrossRef]

213. Ogata, A.; Ito, D.; Mizuno, K.; Kushiyama, S.; Gal, A.; Yamamoto, T. Effect of coexisting components on aromatic decomposition in a packed-bed plasma reactor. Appl. Catal. A Gen. 2002, 236, 9-15. [CrossRef]

214. Ogata, A.; Shintani, N.; Yamanouchi, K.; Mizuno, K.; Kushiyama, S.; Yamamoto, T. Effect of water vapor on benzene decomposition using a nonthermal-discharge plasma reactor. Plasma Chem. Plasma Process. 2000, 20, 453-467. [CrossRef]

215. Mustafa, M.F.; Fu, X.; Liu, Y.; Abbas, Y.; Wang, H.; Lu, W. Volatile organic compounds (VOCs) removal in non-thermal plasma double dielectric barrier discharge reactor. J. Hazard. Mater. 2018, 347, 317-324. [CrossRef] [PubMed]

216. Hirota, K.; Sakai, H.; Washio, M.; Kojima, T. Application of electron beams for the treatment of VOC streams. Ind. Eng. Chem. Res. 2004, 43, 1185-1191. [CrossRef]

217. Zimek, Z. High power accelerators and processing systems for environmental application. Radiation treatment of gaseous and liquid effluents for contaminant removal. IAEA-TECDOC 2005, 1473, 125-137.

218. Son, Y.S.; Kim, J.; Kim, J.C. Decomposition of acetaldehyde using an electron beam. Plasma Chem. Plasma Process. 2014, 34, 1233-1245. [CrossRef]

219. Son, Y.S.; Kim, K.H.; Kim, K.J.; Kim, J.C. Ammonia Decomposition Using Electron Beam. Plasma Chem. Plasma Process. 2013, 33, 617-629. [CrossRef]

220. Han, D.H.; Stuchinskaya, T.; Won, Y.S.; Park, W.S.; Lim, J.K. Oxidative decomposition of aromatic hydrocarbons by electron beam irradiation. Radiat. Phys. Chem. 2003, 67, 51-60. [CrossRef]

221. Hashimoto, S.; Hakoda, T.; Hirata, K.; Arai, H. Low energy electron beam treatment of VOCs. Radiat. Phys. Chem. 2000, 57, 485-488. [CrossRef]

222. Sun, Y.; Chmielewski, A.G.; Licki, J.; Bułka, S.; Zimek, Z. Decomposition of organic compounds in simulated industrial off-gas by using electron beam irradiation. Radiat. Phys. Chem. 2009, 78, 721-723. [CrossRef]

223. Son, Y.S.; Kim, P.; Park, J.H.; Kim, J.; Kim, J.C. Decomposition of trimethylamine by an electron beam. Plasma Chem. Plasma Process. 2013, 33, 1099-1109. [CrossRef]

224. Son, Y.S.; Park, J.H.; Kim, P.; Kim, J.C. Oxidation of gaseous styrene by electron beam irradiation. Radiat. Phys. Chem. 2012, 81, 686-692. [CrossRef]

225. Auslender, V.L.; Ryazantsev, A.A.; Spiridonov, G.A. The use of electron beam for solution of some ecological problems in pulp and paper industry. Radiat. Phys. Chem. 2002, 63, 641-645. [CrossRef]

226. Son, Y.S.; Kim, J.C. Decomposition of sulfur compounds by radiolysis: I. Influential factors. Chem. Eng. J. 2015, 262, 217-223. [CrossRef]

227. Son, Y.S.; Jung, I.H.; Lee, S.J.; Kim, J.C. Decomposition of sulfur compounds by a radiolysis: III. A hybrid system and field application. Chem. Eng. J. 2015, 274, 9-16. [CrossRef]

228. Son, Y.S.; Jung, I.H.; Lee, S.J.; Koutrakis, P.; Kim, J.C. Decomposition of sulfur compounds by radiolysis: II. By-products and mechanisms. Chem. Eng. J. 2015, 269, 27-34. [CrossRef]

229. Kim, J.C. Factors affecting aromatic VOC removal by electron beam treatment. Radiat. Phys. Chem. 2002, 65, 429-435. [CrossRef]

(C) 2020 by the authors. Licensee MDPI, Basel, Switzerland. This article is an open access article distributed under the terms and conditions of the Creative Commons Attribution (CC BY) license (http://creativecommons.org/licenses/by/4.0/). 